\title{
Investigating the impact of the FIC domain Bartonella quintana effector protein, BepA1, on human innate immune responses.
}

by

\section{Aman-Deep Ramela}

Thesis submitted to Victoria University of Wellington in fulfilment for the requirements for the degree of a Master's by Thesis in Molecular Microbiology.

Victoria University of Wellington

2022

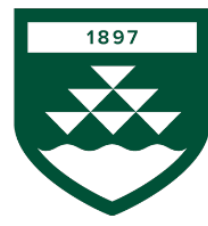

veropan unversirit of 



\section{Abstract}

Bartonella are known as stealth pathogens that subvert the host immune system, using an arsenal of pathogenicity factors that enable them to survive and persist in their reservoir host. The focus of our study is B. quintana, a historically famous pathogen that caused trench fever in soldiers during World War 1, but today causes an increasing number of urban trench fever cases in vulnerable populations, such as homeless or immunocompromised groups.

After exposure to B. quintana, the bacteria traverse the skin and enter the bloodstream, infecting erythrocytes. B. quintana encounter host immune cells in the vascular system, but are not killed, hijacking these cells to disseminate into the lymphatic system where they eventually make their way into the bloodstream. How B. quintana modulates host immune responses to its favour during early infection is very poorly understood due to the current lack of research surrounding this pathogen.

B. quintana, like many Bartonella species, possesses a Type IV Secretion System, a large protein complex that is used to inject Bartonella Effector Proteins, Beps, directly into target host cells. B. quintana encodes six BEP proteins, of which BepA1 and BepA2 are the best studied. BepA1 contains a FIC domain, which in other pathogens have been shown to catalyse addition of an AMP moiety to target host proteins, interrupting function and downstream signalling. This process, AMPylation, is used by various bacterial pathogens to interfere with actin cytoskeletal signalling to inhibit phagocytosis. However, the AMPylation function in $B$. quintana BepA1 remains to be shown. BepA2 has been shown to inhibit apoptosis once translocated into endothelial cells.

Previous studies in our lab indicated that BepA1 may play a role in suppression of the innate immune system. Wild type BepA1-transfected HeLa-229 cells showed decreased levels of IL-6 and IL-8 in comparison to an empty vector control. We thus hypothesised that BepA1 is being translocated into host cells to AMPylate a signalling protein/s to suppress immune signalling and immune cell recruitment, enabling the survival and persistence of B. quintana in the bloodstream.

Our study thus focused on better understanding the host-pathogen interactions between B. quintana and the human innate immune response. We began by broadly characterising the innate immune responses of human cells transfected with the wild type B. quintana BepA1 protein. HeLa-229 cells were transfected with a vector expressing the BepA1 wild type protein, or a catalytically dead BepA1 mutant protein. Cells were stimulated with TNF- $\alpha$, and cytokine 
and chemokine expression was evaluated. These observations were validated via qPCR analysis of the transfected, TNF- $\alpha$ treated cells.

Lastly, we aimed to generate a targeted BepA1_BepA2 B. quintana deletion mutant to further study the consequences on host-pathogen interactions. Unfortunately, we were unable to generate this mutant.

Our work provides the first evidence that we know of that suggests a bacterial effector with a FIC domain is involved in manipulation of cytokine and chemokine expression. Our work provides a foundation for a better understanding of the early stages of Bartonella infection and pathogenesis. With this research, we hope to shed light on how B. quintana hampers immune signalling during infection, leading to its intracellular persistence and survival in the reservoir host. 


\section{Acknowledgements}

Before anyone else, I owe my deepest gratitude to my supervisor, Dr. Joanna MacKichan. It was in my final year of undergraduate lectures when I had the opportunity to take a course you were teaching. That very course was the one where I decided microbes and research were what I wanted to dedicate my life to. Two and a half years ago I approached you and asked if I could be a part of your lab. You agreed, trusting me with this project, taking me in, teaching me the secrets of microbiology; guiding and encouraging me when experiments kept failing. Your kindness has made my time in your lab most memorable. I came in hopeful and eager to learn, and I leave confident, more knowledgeable and surer than ever, that this is the path for me. Thank you.

To my lab partner, Alvey. I remember when I first met you. It was in the microbiology lab where you had that colourful funky lab coat. I remember thinking, I wish I that lab coat. I still don't have that lab coat but instead I have gained a friend. Thank you for taking me under your wing and teaching me the art of the Western Blot, the magic of cell culture and transfections and for proof-reading many, many drafts of my thesis. Thank you for showing me cute pictures of animals of which were mostly of cats, and last but most importantly, thank you for always saying, “Everything will be alright” even when it wasn't. This line I will carry with me always.

To Katharina, the resident qPCR whiz of AMAC, thank you for being so ready and willing to help me with qPCR. Your knowledge, patience and guidance are what made the then daunting technique of qPCR much, much easier. Thank you for talking me through qPCR data analysis and for providing me with tips for publishable results. When I approach qPCR with a confident mind in the future, know it is all because of you.

Lastly, to my family, who have been ever so patient through these last few years. Thank you for your understanding when my research turned early mornings into late nights, and long weekends. In particular, to my sister, my confidant, my rant buddy and fellow cat enthusiast, thank you for the giggles, the food and the fun times amongst the stressful times. When it is your turn, know that I will do the same. 


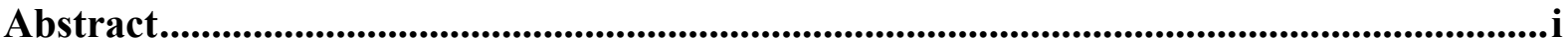

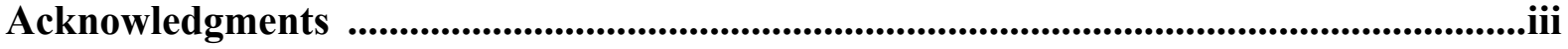

Table of Contents .........................................................................................................................................iv

List of figures............................................................................................................................................. vi

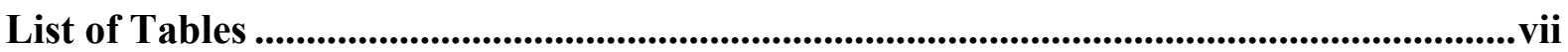

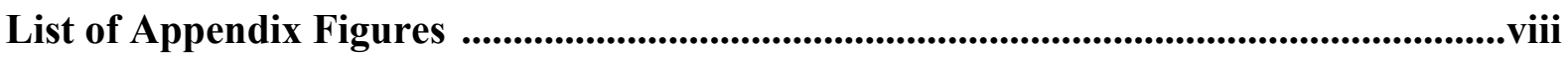

Abbreviations ................................................................................................................................ ix

Chapter 1 - Introduction .......................................................................................................................1

1.1 The Clinically relevant Bartonella species for Humans...............................................2

1.1.1 Bartonella bacilliformis........................................................................................ 2

1.1.2 Bartonella henselae - a zoonotic disease ........................................................... 3

1.1.3 Bartonella quintana - an ancient bacterial pathogen ............................................. 4

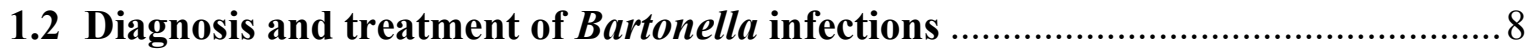

1.3 Life cycle and infection strategy of Bartonella .............................................................8

1.4 Evasion of innate immunity by Bartonella in mammalian hosts ............................. 12

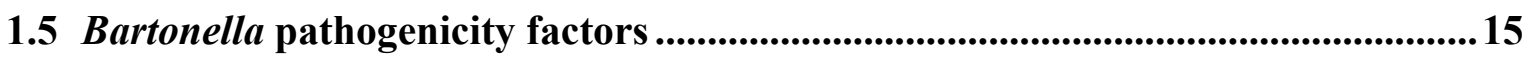

1.5.1 T4SS - Evolution, structure and function $\quad$................................................15

1.5.2 Bartonella gene transfer agents..............................................................18

1.5.3 Bartonella type 4 secreted effector protein structure and function...........20

1.6 The FIC domain ........................................................................................................................23

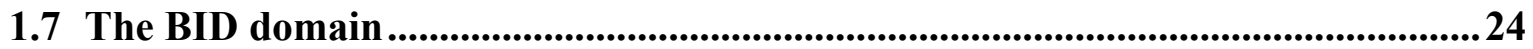

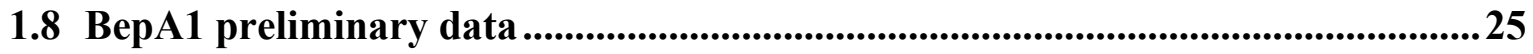

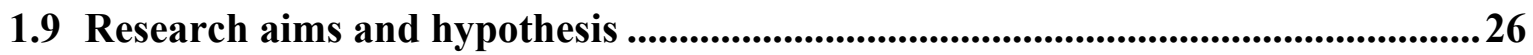

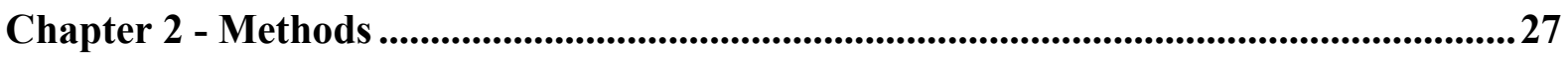

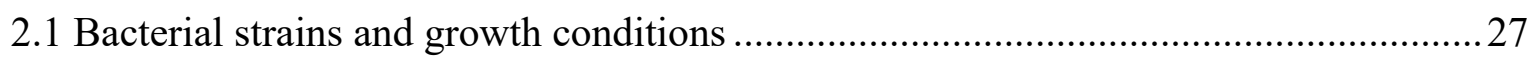

2.2 Bacterial plasmids ..........................................................................................................28

2.3 Media recipes .....................................................................................................................29

2.4 Standard Molecular Biology Techniques........................................................................31

2.5 Construction of the BepA1 mutant mammalian expression vector........................34

2.6 Construction of the BepA1_BepA2 mutagenic plasmid .............................................34

2.7 Cell Culture and Transfection ..................................................................................35

2.8 Gene expression analysis ......................................................................................38

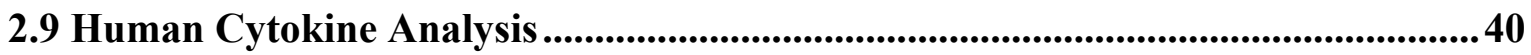


Chapter 3 - BepA1 and Cytokine and Chemokine Expression in HeLa cells...................44

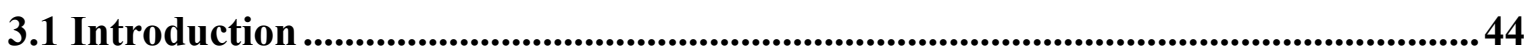

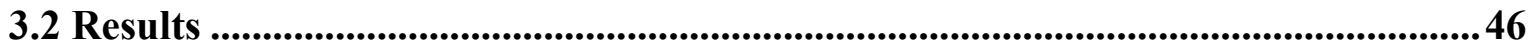

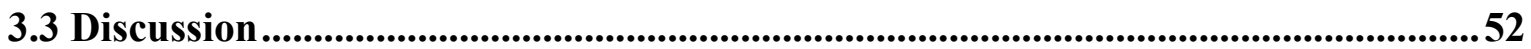

Chapter 4 - Reverse Transcriptase and Quantitative PCR ...........................................56

4.1 Introduction .............................................................................................................................56

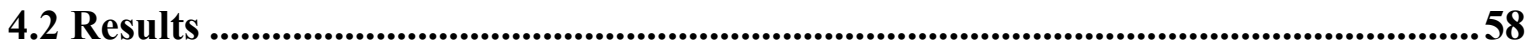

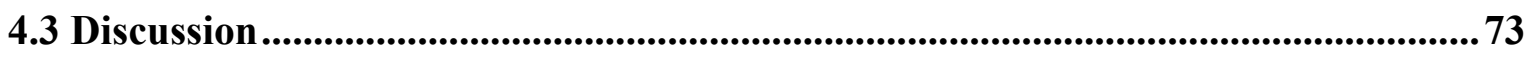

Chapter 5 - Generation of a BepA1_BepA2 B. quintana deletion mutant .......................74

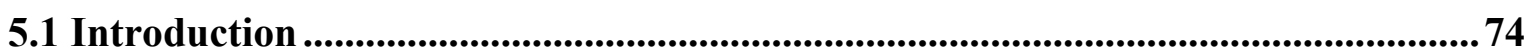

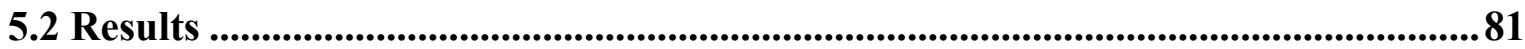

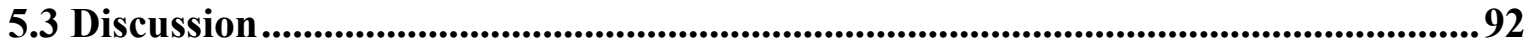

Chapter 6 - Research Motivations, Key Findings and Future Directions........................95

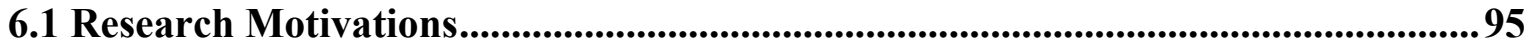

6.2 Key Findings ......................................................................................................................96

6.3 Future Research ............................................................................................97

6.4 Concluding remarks........................................................................................100

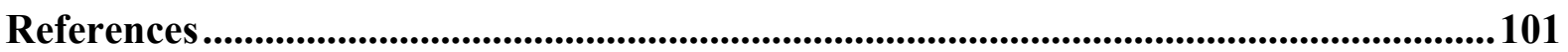

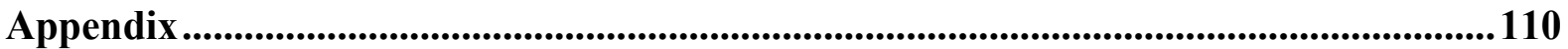




\section{List of Figures}

Figure 1.1 Recovery of 4000-year-old Bartonella quintana DNA........................5

Figure 1.2 Efforts during World War I to deal with the body louse vector for B. quintana...6

Figure 1.3 The hypothesised infection strategy of reservoir host infections by $B$. henselae...11

Figure 1.4 Hypothesised model of $B$. quintana LPS. ................................. 13

Figure 1.5 Current models for the T4SS pilus biogenesis and substrate translocation.........17

Figure 1.6 The BaGTA and DNA transfer cycle..................................... 19

Figure 1.7 The virB/virD4/Bep pathogenicity island of $B$. henselae and B. quintana.......21

Figure 3.1 Proteome Profiler Human Cytokine Array exposed membranes for cell medium......................................................................... 47

Figure 3.2 Average pixel intensity values for all 36 cytokines and chemokines tested in all four cell samples $\quad$ for $\quad$ cell medium

Figure 3.3 Average pixel intensity values for all 36 cytokines and chemokines tested in all four cell samples for cell

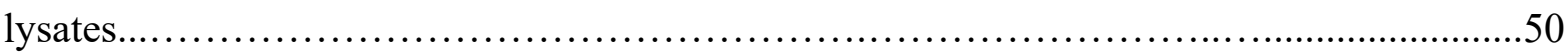

Figure 3.4 Mean pixel intensities of selected cytokines and chemokines in BepA1 WT and FIC domain mutant TNF- $\alpha$ treated cell medium samples, and cell lysates...................... 51

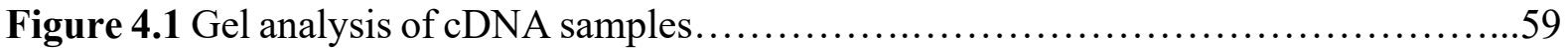

Figure 4.2 Gel analysis of PCR of reference gene, Cyclophilin A........................60

Figure 4.3 Gel analysis of PCR for IL-6, IL-8, and SM22 ...........................61

Figure 4.4 Amplification plots for Cyclophilin A, IL-6, IL-8 and SM22 .................64

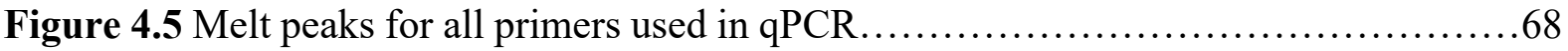

Figure 4.6 The normalised expression ratios for target genes IL-6, IL-8 and SM22 using the

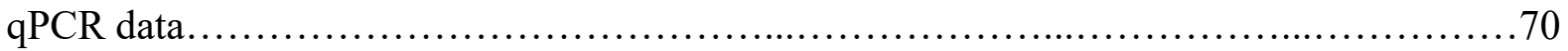

Figure $4.7 \mathrm{Gel}$ analysis of Myozap..................................................

Figure 5.1 B. quintana str. Toulouse T4SS structural and effector genes....................75

Figure 5.2 BepA1 and BepA2 nucleic acid sequences of B. quintana Toulouse strain

Figure 5.3 The SacB mutagenesis strategy used to construct the BepA1_BepA2 B. quintana deletion mutant. .79

Figure 5.4 PCR products of surrounding sequence of BepA1 and BepA2 ....................81

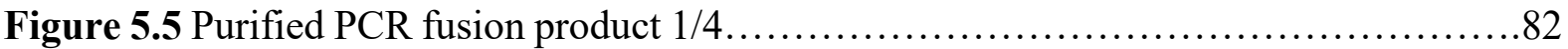


Figure 5.6 Digest of the PCR fusion product $1 / 4$ and $\mathrm{pEX} 18 \mathrm{Gm}$ vector.

Figure 5.7 Gel analysis of the ligations of the PCR fusion product $1 / 4$ and the pEX18Gm vector samples 84

Figure 5.8 Electroporated E. coli (10G E. cloni) cells expressing the pEX18Gm_PCR fusion product $1 / 4$ .86

Figure 5.9 Agarose gel image of purified and digested plasmid from 10G E. cloni cells

Figure 5.10 Plate containing colonies for successful bacterial mating between E. coli pACYC184 and S17-pEX18Gm_PCR fusion product 1/4 88

Figure 5.11 Triparental mating strategy. .93

\section{List of tables}

Table 2.1 Bacterial strains used in this study with their respective strain characteristics and source .27

Table 2.2 Bacterial plasmids use in this study with their characteristics...................28

Table 2.3 Primers used in this study for generating the BepA1 mutagenesis plasmid........31

Table 2.4 Primers used in this study for RT-PCR and RT-qPCR

Table 2.5 PCR conditions used for generating the preliminary BepA1 and BepA2 PCR products for the mutagenesis plasmid, and the BepA1_2 mutagenesis PCR product...........32

Table 2.6 Optimised annealing and extension times and temperature for RT-PCR ............39

Table 4.1 Nanodrop concentrations, 260:280 and 260:230 ratios of RNA samples..........58

Table 4.2 Nanodrop concentrations, 260:280 and 260:230 ratios are summarised for all cDNA samples 


\section{List of Appendix Figures}

Appendix Figure 7.1 Pairwise alignment of BepA1 WT protein and the BepA1 FIC domain mutant DNA sequence.

Appendix Figure 7.2 Pairwise alignment of BepA1 WT protein and the BepA1 FIC domain mutant protein amino acid sequence.

Appendix Figure 7.3 Proteome Profiler Array Membrane Coordinates

Appendix Figure 7.4 Proteome Profiler Array Exposed Membranes - Cell mediums and Cell lysates....

Appendix Table 7.5.1 Average pixel intensity values for HeLa cell mediums samples.

Appendix Table 7.5.2 Average pixel intensity values for HeLa cell lysates samples. 115

Appendix Figure 7.6 qPCR raw data and Livak analysis calculations.... 116

Appendix Figure 7.6.1 qPCR raw data and Li vak analysis calculations are presented for IL6.

Appendix Figure 7.6.2 qPCR raw data and Livak analysis calculations are presented for IL-8 118

Appendix Figure 7.6.3 qPCR raw data and Livak analysis calculations are presented for SM22 119

Appendix Figure 7.7 Sequencing of the BepA1_BepA2 mutagenesis plasmid insert.... 120 


\section{Abbreviations}

AMP of AMPylation - Adenosine Monphosphate

AP-1 - Activating Protein-1

BaGTA - Bartonella Gene Transfer Agent

BEPs - Bartonella Effector Proteins

BID - Bartonella Intracellular Domain

CatMut - Catalytic mutant

chIP - Chromatin Immunoprecipitation

cIAP - calf intestinal alkaline phosphatase

CSD - Cat Scratch Disease

CTL1 - Cytotoxic T Lymphocyte

$\mathbf{C}_{\mathbf{T}}$ - Threshold Cycle

cyclA - Cyclophilin A

CCL2/MCP-1 - Monocyte Chemotactic Protein-1

CCL5/RANTES - Regulated upon Activation normal T cell expressed and secreted

CXCL/GRO $\alpha$ - Growth Related Oncogene- $\alpha$

CXCL10/IP-10 - Interferon gamma-induced protein 10

$\mathbf{d d H}_{2} \mathbf{O}$ - double distilled $\mathrm{H}_{2} \mathrm{O}$

dsDNA - double stranded DNA

FCS - Foetal Calf Serum

FIC - Filamentation Induced by cAMP

GTA - Gene Transfer Agent

HGT - Horizontal Gene Transfer

ICAM-1 - Intercellular Adhesion Molecule 1

IL-6 - Interleukin 6

IL-8 - Interleukin 8

LB - Lysogeny Broth

LPS - Lipopolysaccharide

MIF - Macrophage Inhibitory Factor

MRIP - myosin phosphatase-RhoA interacting protein

MRTF-A - Myocardin Related Transcription Factor-A

NFкB - Nuclear Factor kappa (к) B

NLRs - Nucleotide-binding oligomerization domain-like receptors

ori - Origin of Replication

oriT - Origin of Transfer

PAMPs - Pathogen Associated Molecular Patterns

PBS - Phosphate Buffered Saline

PRR - Pattern Recognition Receptors

qPCR - quantitative PCR

RcGTA - Rhodobacter capsulatus Gene Transfer Agent

RT-PCR - Real-Time-PCR

Serpin E1 / PAI-1 - Plasminogen Activator Inhibitor-1

SRF - Serum Response Factor

TAA - Trimeric Autotransporter adhesins

TLRs - Toll Like Receptors

TNF- $\alpha$ - Tumour Necrosis Factor- $\alpha$

T4SS - Type IV secretion system

VOMPs - Variably expressed Outer Membrane Proteins

WT - Wild type 


\section{Chapter 1 Introduction}

The genus Bartonella describes a group of emerging Gram-negative, blood-borne alpha-proteobacterial zoonotic pathogens $[1,2]$. Alpha-proteobacteria are one of the most diverse bacterial subdivisions, often exhibiting extreme variations in lifestyle, geographical distribution and genome size [1]. Most are associated with plant or animal hosts. Species belonging to the Bartonella genus are prevalent worldwide; they are extremely diverse and are associated with a large number of mammalian species. Studies have shown that over $60 \%$ of infectious diseases that emerged between 1940 and 2004 are zoonotic and a staggering 54.3\% of these infections are of bacterial origin [3].

To date, there are more than 30 known species and subspecies of Bartonella, that have been described and isolated from nearly all tested mammalian species including cats, dogs, humans, kangaroos and otters [4-6]. Of these 30 species and subspecies of Bartonella, 17 have been reported to cause human disease, with three that are significant to human health because of the profound disease they can cause. These species are: Bartonella bacilliformis, Bartonella henselae and Bartonella quintana [7, 8]. A wide range of mammalian hosts are infected with Bartonella species, each one specialised to one or a couple specific mammalian reservoir hosts and their cognate blood-feeding ectoparasite. It is in their reservoir host that Bartonella spp. have the potential to cause debilitating chronic illness. Bartonella are also able to cause incidental infection in non-reservoir hosts but the disease often remains acute.

Bartonella spp. are primarily vector-borne bacteria and are transmitted between reservoir hosts through a variety of blood-feeding arthropod ectoparasite vectors, such as flies, ticks, fleas and lice [9]. Zoonotic infections, by contrast, frequently occur via animal bites or scratches.

Species in the Bartonella genus, despite their widespread geographic distribution and association with a range of mammalian species, can be divided into three major groups, designated $\mathrm{A}-\mathrm{C}[10]$. These groups have been assigned based on phylogenetic and sequence analyses of multiple Bartonella genomes. Briefly, group A includes ruminant adapted Bartonella species. Group B includes species isolated from very small mammals such as rats and squirrels, while group $\mathrm{C}$ includes species isolated from rodents, cats and dogs [10]. $B$. quintana belongs to group $\mathrm{C}$.

Bartonella spp. are unusual bacteria in that they infect a wide range of mammalian hosts with a very diverse worldwide prevalence. These bacterial pathogens have a complicated but highly successful infection strategy, beginning their life cycle in the gut of the blood-feeding 
arthropod vector, followed by persistence in a vascular niche in their respective mammalian reservoir host. From this niche, the bacteria periodically invade the host's erythrocytes, circulating intracellularly until possibly being taken up in another blood meal [11]. Bartonella have been proposed to invade other vascular cells but the current experimental data does not support this hypothesis.

Bartonella species infection of their reservoir mammalian host may result in an acute infection or a chronic bacteraemia. Clinical presentations range from mild to severe. A major question in the field is, how do Bartonella survive and persist, even for months or years, in the host bloodstream, where they are susceptible to numerous host immune recognition mechanisms?

In the bloodstream, Bartonella spp. invade erythrocytes, facilitating their own transmission back to their arthropod vector, completing their life cycle. This parasitisation of red blood cells is unique among bacterial pathogens and is only otherwise seen in malarial infections. Colonisation of the host's erythrocytes is essential for long-term infection of the reservoir host, and transmission of Bartonella spp.

Bartonella research is rich in questions: how do these bacteria survive in host erythrocytes without causing a devastating haemolytic reaction? What kinds of host-bacterial interactions are occurring that allow these pathogens to persist in the bloodstream without eliciting septic shock or an overwhelming inflammatory response? There is still much to learn about the molecular pathogenesis of Bartonella infections, and how these blood-borne stealth pathogens so efficiently and competently invade and reside in their reservoir hosts.

\subsection{The Clinically relevant Bartonella species for Humans}

\subsubsection{Bartonella bacilliformis}

B. bacilliformis is a potentially deadly pathogen, in contrast to other species of the genus. It is spread via the bite of the infected sand fly, Lutzyomia verrucarum, which has a limited geographical habitat in the Andes Region of South America. This geographical isolation is why B. bacilliformis infections are restricted and endemic to the Andes region [12]. B. bacilliformis was described in 1909 by the Peruvian physician, Alberto Barton, when he observed the presence of intraerythrocytic bacilli in blood smears of patients suffering from Carrion's disease [13]. Carrion's disease is biphasic and can result in acute disease, Oroya fever with high mortality, or a chronic disease, verruga peruana [14]. During acute infection, the 
patient will experience a haemolytic anaemia with temporary immunosuppression if untreated. The chronic stage of infection usually occurs following repeated exposure, in areas of endemicity. In the chronic infection, verruga peruana, or Peruvian warts, emerge as nodular skin lesions that may cause pruritis or unexpectedly bleed. These lesions, frequently seen in children, occur when bacteria have invaded endothelial cells, and have a lower morbidity than the acute phase [14].

Previously the term bartonellosis was used to describe the specific disease caused by $B$. bacilliformis, but now generally describes the wide range of human diseases, many not as severe as Oroya fever, caused by multiple Bartonella spp. Bartonellosis can involve any combination of fever, vascular skin lesions, bacteraemia, feelings of malaise and lymphadenopathy. However, many of these symptoms have been mainly associated with immunocompromised people [14].

\subsubsection{Bartonella henselae - a zoonotic disease}

$B$. henselae is the species that most commonly infects humans. B. henselae is the causative agent of cat scratch disease (CSD). Cats are the reservoir host for $B$. henselae, and humans are an incidental host. $B$. henselae is spread between cats via the bite of the infected cat flea, Ctenocephalides felis [15]. Although humans can be bitten by infected cat fleas, there is no evidence that humans become infected with $B$. henselae this way. While it has been reported that people have acquired $B$. henselae infections from tick bites, this remains controversial and debated within the field [16]. CSD is a fairly common disease and was first described in Paris in 1950 as "maladie des griffes du chat" by Dr. Robert Debré [17]. In the early 1990s, a clear link was finally established: Regnery and colleagues from the CDC, cultured $B$. henselae from blood samples of an HIV-infected individual and the examination of the blood cultures, including analyses of fatty acid synthesis and 16S RNA gene sequence, revealed that the organism was closely related to Rochalimaea quintana, the agent of trench fever; the new bacterium was thus named Rochalimaea henselae [18]. A few years later, the genetic similarities with Bartonella bacilliformis were noted and the three species were combined into the Bartonella genus [18].

Humans infected with CSD will most commonly experience chronic lymphadenopathy and the infected node is described as "warm, tender and erythematous". Fever can also occur as well as fatigue, malaise and headache, although these symptoms are generally mild [19]. 
Epidemiologically, B. henselae is associated with CSD in otherwise healthy individuals, with bacillary angiomatosis or endocarditis primarily occurring in immunocompromised patients.

\subsubsection{Bartonella quintana - an ancient bacterial pathogen}

B. quintana is spread via the bite of the infected body louse, Pediculus humanus corporis, and as with B. bacilliformis, humans are the main reservoir [20]. B. quintana was identified as a significant pathogen during World War I, although evidence suggests that it has infected humans for far longer. B. quintana DNA was identified in 4000-year-old dental pulp from a human skeleton in France, seen in Figure 1.1 below, suggesting it is an ancient bacterial disease in humans [21]. In more recent times, B. quintana infection has been associated with war, upheaval, and poverty. B. quintana DNA was isolated from dental pulp from mass graves of soldiers in the 1700's War of Spanish Succession [21]. B. quintana also afflicted soldiers in Napoleon's Grand Army as they sought retreat from Russia in the early 1800's [22, 23]. B. quintana however, is most famous for causing relapsing trench fever during World War I, where it infected more than 1 million German and Allied troops, rendering each infected soldier unfit for at least 60 days [24].

Trench fever is the manifestation of nonhemolytic intraerythrocytic bacteraemia in acute infection, but B. quintana can also cause chronic infections and further complications, including endocarditis and vasoproliferative skin lesions in immunocompromised patients [25]. Trench fever, as it became known to Allied doctors from 1915, is also known as five-day fever and less commonly as Volhynian fever, the name it was given by a German doctor in 1916 [24]. British troops were responsible for dissemination of the disease as they carried it with them to Mesopotamia and Salonika during World War I [24]. The first medical officers that tried to treat trench fever used a wide variety of ineffective treatments, including mercury, methylene blue and trypan red [26]. The most important preventative actions taken against trench fever in World War I were the introduction of delousing practises to get rid of the body louse, such as treating uniforms and bedding with steam, e.g., through the development of the Foden Thresh steam disinfector, as seen in Figure 1.2,. Chemical insecticides, such as DTT, were developed and used widely in World War II, before the dangers of this compound were known. This led to a reduced impact of louse-borne diseases; after the war, improvements in hygiene and living conditions greatly aided the control of B. quintana infections. 


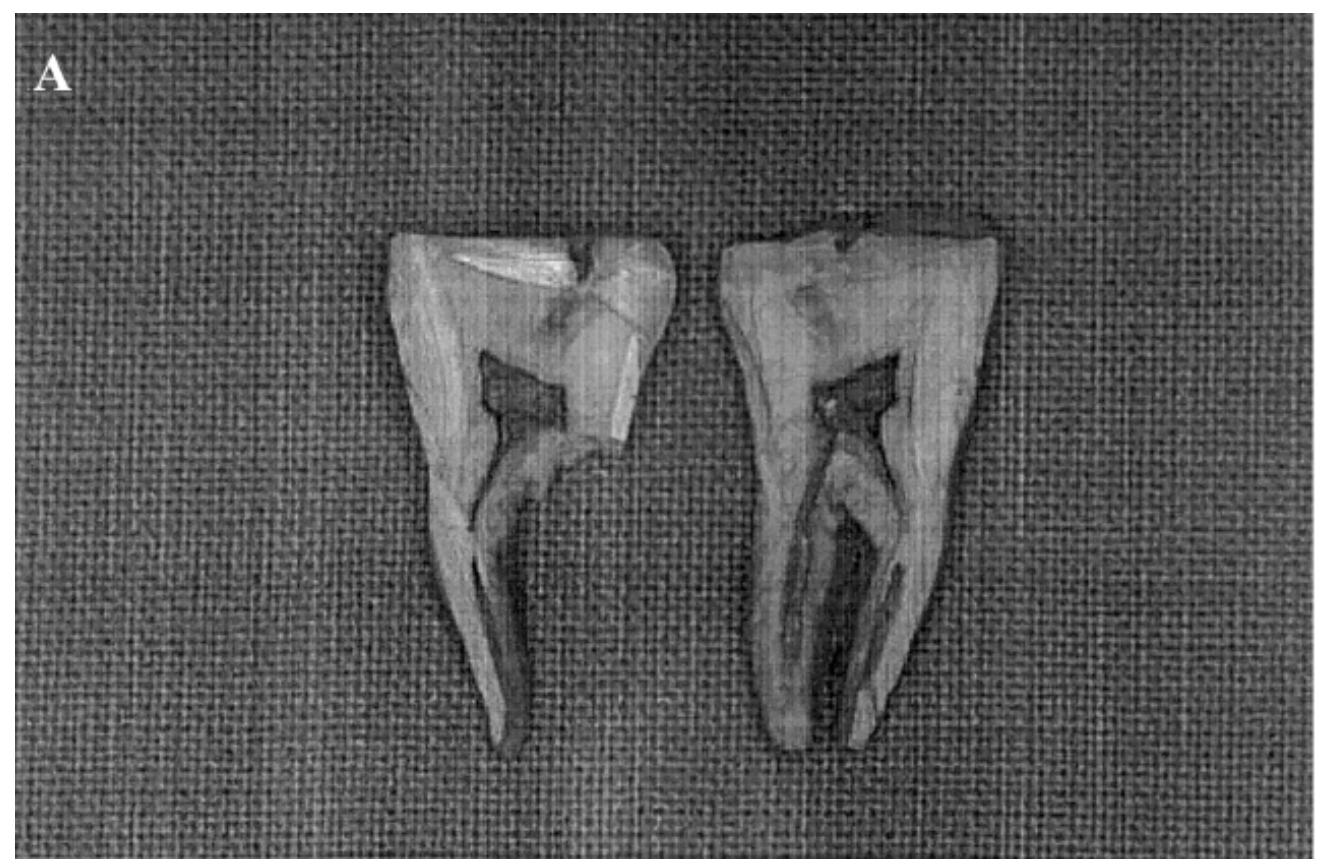

Figure 1.1: Recovery of 4000-year-old Bartonella quintana DNA. The premolar was collected from a 4000-year-old skeleton from which $B$. quintana DNA was obtained from the dental pulp. PCR amplification reactions and sequencing yielded 2 fragments of $B$. quintana DNA. Reproduced with permission from the Oxford University Press. Drancourt et al., 2004 [21].

The earliest report of trench fever is from 1916, by McNee et al., who demonstrated that the causative agent of this disease was found in the blood [27]. This same team was the first to propose that lice may be involved in the transmission of trench fever. To test this hypothesis, McKee and colleagues scratched the skin of volunteers and rubbed louse faeces into the wound. Through their experiment, they were able to show that the disease is transmitted through scratching of the louse's infectious faeces into the derma, and not by the louse bite [27]. These preliminary experiments were followed by the British Army commissioning of the Trench Fever Investigation Committee, led by Sir David Bruce. This committee carried out, over two years, a series of experiments on volunteers that shed light on the transmission and course of the infection [28]. The organism, then known as Rickettsia quintana, was seen in the excreta of infected lice in the WWI era, but was not cultured until the 1960s [29]. 

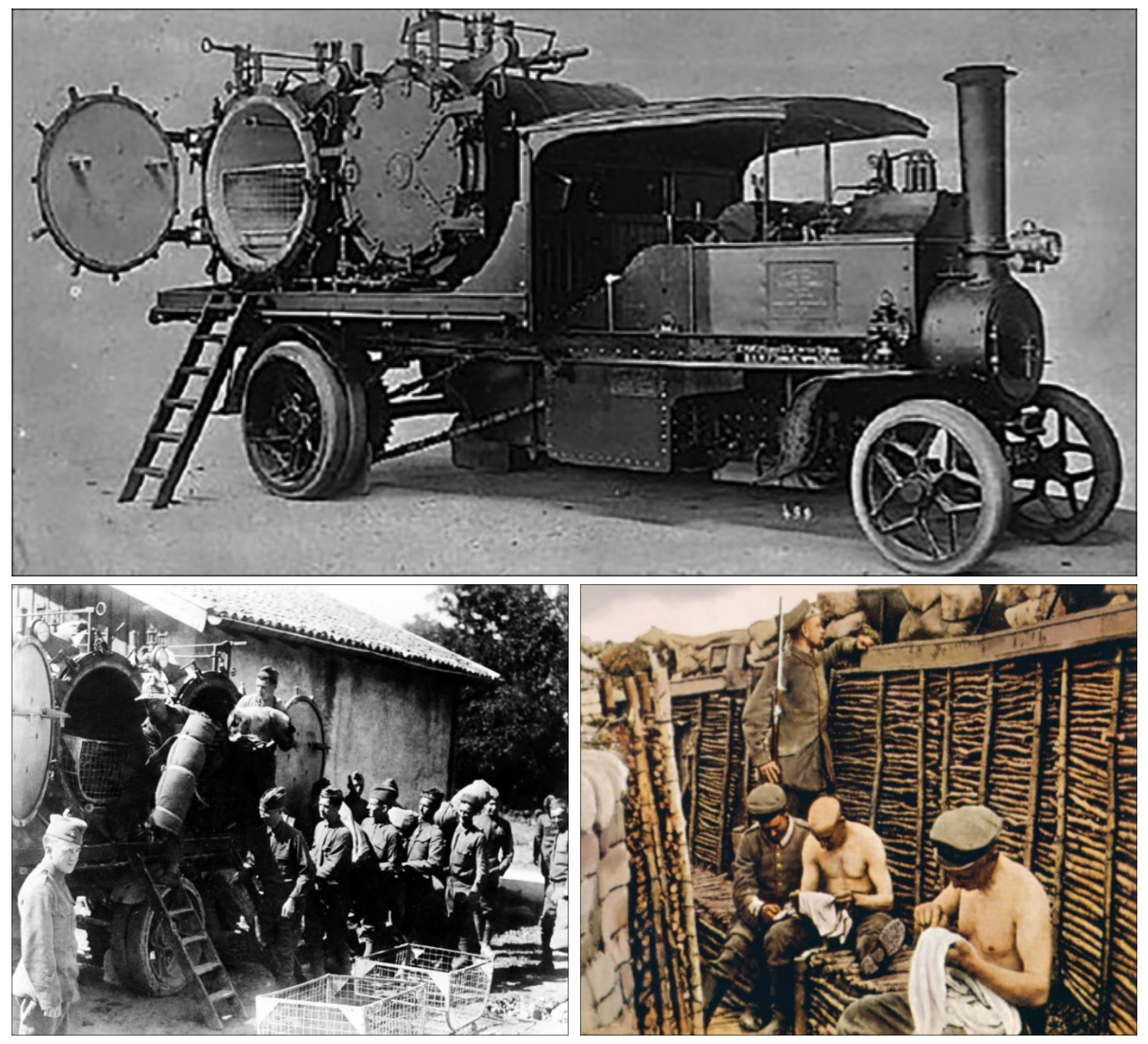

Figure 1.2 Efforts during World War I to deal with the body louse vector for B. quintana. Top photo: The Foden Thresh steam disinfector, used to steam soldiers' uniforms. Bottom left: The Foden Thresh steam disinfector in use. Bottom right: soldiers in a trench manually picking lice and eggs from their uniforms. Reproduced with permission from Elsevier. Anstead., G.M. 2016 [24].

Human volunteer studies, to fulfil Koch's postulates for trench fever and B. quintana, were carried out following the successful culture of the organism, as the disease could not be reproduced in animals. J.W. Vinson and his team were the first to successfully isolate a Mexican strain of Rickettsia quintana from a volunteer trench-fever patient and culture this bacterium on blood agar [29]. Vinson then inoculated three volunteers with $R$. quintana, finding that typical clinical trench fever was induced in two of the three volunteers, as the third volunteer withdrew from the study [29]. The etiology of both volunteers' clinical infection was confirmed through xenodiagnoses and recovery of the pathogen from the peripheral blood, satisfying all of Koch's postulates for the disease [30]. In the post-war period, trench fever largely fell below detectable levels for many years. The absence of large-scale infection of 
troops by B. quintana meant interest and research into this pathogen dropped dramatically. But B. quintana re-emerged as a significant opportunistic infection during the AIDS epidemic, when it became known as urban trench fever. Urban trench fever is associated with an increased likelihood of louse infestation, usually involving homelessness, alcoholism and general poor conditions of life [30]. Towards the end of the $20^{\text {th }}$ century, numerous reports emerged about B. quintana infections in homeless or alcoholic populations in Seattle, San Francisco, Marseilles, France and elsewhere [31-34].

These infected individuals were both HIV positive and negative and exhibited various clinical presentations of the B. quintana infection. Many of these reported infections were confirmed to be due to $B$. quintana but the researchers did not rule out infections due to other species [31].

More recently, there have been reports of a cluster of B. quintana infections among the homeless communities in Seattle and Denver. In Seattle three homeless individuals tested positive for B. quintana infection, where two had an infection of the heart valves and one a blood stream infection $[35,36]$. Three cases have also been reported in Denver in 2020 , raising concerns about public health and homeless encampments [37].

Manifestations of Bartonella infections in patients with HIV infections are characterised by bacillary angiomatosis, bacillary peliosis hepatis, a closely related angioproliferative lesion of the liver and spleen and bacteraemia, or a combination of all three [38]. The first case of bacillary angiomatosis due to B. quintana infection was described in 1983, at the beginning of the AIDS epidemic, by Stoler et al., as "an atypical cutaneous infection associated with acquired immune deficiency syndrome" [39]. There were many reports of HIV patients who developed subcutaneous nodules with $B$. henselae and B. quintana identified as the causative agents in 1990 and 1992 [38, 40]. Treatments for Bartonella infections, generally erythromycin or doxycycline, were subsequently developed.

Individuals infected with B. quintana typically experience a mild to severe fever, which may be relapsing or of long duration, as well as headache and pain in the long bones of the legs; however, some individuals remain asymptomatic. One rare but severe complication of $B$. quintana infection is infective endocarditis. This occurs when the bacteria colonise the inner layer of the heart, causing inflammation and destruction of heart valves [41]. B. quintana endocarditis is characterised by mononuclear cell inflammation, extensive fibrosis, large calcifications and small vegetations [42].

With the resurgence of $B$. quintana infections, research involving this stealth pathogen has become increasingly important for medicine. 


\subsection{Diagnosis and treatment of Bartonella infections}

Many cases of $B$. quintana infection can resolve spontaneously without treatment, as was seen in World War I [26]. In immunocompromised individuals, however, untreated $B$. quintana and $B$. henselae infections can lead to debilitating illness and can even be fatal [43]. These infections can have a variety of manifestations and localise to a range of tissues so there is no single treatment for all Bartonella-associated diseases. Furthermore, not all bartonellosis infections require treatment. For example, CSD is usually a self-limiting infection which can resolve without antibiotic assistance, whereas infection with $B$. bacilliformis is more serious and requires medical intervention. Thus, treatment options are tailored to the infecting species and the clinical situation [44]. Diagnosis often involves a combination of culture, serological or nucleic acid amplification techniques, with serology most commonly used. Serology and nucleic acid amplification tests can be used in combination for diagnosis, but both have low sensitivity and specificity which results in both high false positive and negative rates [45]. The most common method of diagnosis of endocarditis caused by Bartonella involves using an indirect immunofluorescence assay to identify antibodies to the bacterium, but PCR can also be used to identify Bartonella DNA in the patient's blood or resected heart valves [46].

Combination antibiotic therapy has been shown to be effective against intraerythrocytic bacteraemia. Bacteraemia caused by $B$. quintana is treated with a combination of gentamicin and doxycycline [44]. Infective endocarditis is also treated with a long course of the antibiotics gentamicin and doxycycline, and sometimes patients require surgery to repair or replace damaged heart valves [44]. When a patient exhibits specific risk factors, such as urban homelessness, with a non-localising febrile illness with unknown cause, B. quintana infection should be considered as a diagnosis.

\subsection{Life cycle and infection strategy of Bartonella}

The main mode of transmission for the reservoir host is the arthropod vector, although some Bartonella reservoir host infections may begin with a bite or scratch from an infected animal, with details of the infection cycle differing between species.

In B. quintana infection, the body louse takes a blood meal and simultaneously deposits faeces, which contains bacteria, on the host's skin [11]. However, not all biting arthropods deposit faeces at the site of the blood meal in a Bartonella infection; others inject saliva or draw blood, leading to direct inoculation with Bartonellae. The louse bite causes irritation, which triggers scratching and inoculation of the insect's bacteria-containing faeces into the 
host's derma [11]. The bacteria then take residence in the host's derma, i.e., the 'dermal niche' [47]. Eventually, via the lymphatics and using mechanisms of immune evasion that remain unclear, the bacteria reach an enigmatic "blood seeding niche", a site within the bloodstream where the bacteria can persist but emerge to infect erythrocytes periodically. Even when Bartonellae are inoculated into the bloodstream, there is a delay of 5-7 days before they appear inside erythrocytes, suggesting that they reprogram and replicate in a vascular site. The "blood seeding niche" has been proposed to be an intracellular niche inside vascular endothelial cells, but this remains a subject of debate in the absence of published evidence from natural or laboratory infections $[48,49]$.

Endothelial cells are implicated because they are the primary components of the bacillary angiomatosis lesions seen in AIDS patients. In the lab, Bartonella spp. readily invade endothelial cells in vitro, but there is limited evidence that the bacteria invade endothelial cells in the host, despite numerous careful studies of human biopsies and lab animals [49-51]. It is suggested that Bartonella need to reside in an intracellular niche to be protected from the host immune response, but additional, unknown, mechanisms of immune evasion may be important.

A careful study published by Chiaraviglio et al investigated an infection of a rodentborne species, Bartonella taylorii, in an immunodeficient mouse as a model for human pathologies [48]. These researchers used multiple microscopy techniques to demonstrate that B. taylorii is found in extracellular aggregates embedded within the collagen matrix of the vascular endothelium. This is similar to the observation of biopsies in CSD and bacillary angiomatosis. Furthermore, despite the overwhelming infection in the mouse, there was no evidence of intracellular replication in endothelial cells [48]. The predominant surface molecules on B. quintana, the Variably expressed Outer Membrane Proteins, VOMPs, bind to components of the extracellular matrix, so the bacteria may remain embedded and hidden from immune cells in the extracellular matrix of the adventitia [52].

Recently, a study by Hong et al. used a rat model to show how B. tribocorum bacteria are able to reach the bloodstream following dermal inoculation [53]. In most bacterial infections, the pathogen is restricted to the inoculation site or is drained into the adjacent lymph nodes, resulting in inflammation and pathogen clearance. B. tribocorum, by contrast, was shown to evade phagocytosis; following dermal inoculation, a small number of bacteria were engulfed by rat peritoneal macrophages but pyroptosis was inhibited by the bacteria, blocking inflammatory amplification. Following the escape of clearance by innate immunity, $B$. tribocorum was shown to invade lymphatic circulation and subsequently enter the bloodstream, with intraerythrocytic infection detected several days later [53]. This finding sheds light on 
how Bartonella infections may develop, initiating in the derma and reaching the bloodstream via the lymphatic system. Infection caused by B. tribocorum differed from Salmonella infection of the lymph nodes, which resulted in inflammation, blockage of efferent lymphatic vessels and enlargement of the infected lymph nodes, known as lymphadenopathy. B. tribocorum infection in rats, however, failed to cause significant lymphadenitis at an early stage of the infection [53]. The precise mechanisms by which Bartonella species evade innate immunity remain unknown.

After Bartonella organisms are introduced into a new host through a blood meal of an infected arthropod, intraerythrocytic infections can be detected after a few days. After Bartonella spp. invade host erythrocytes, they replicate for a limited number of cell divisions [54]. The bacteria then persist in the red blood cell for its remaining lifespan, as it continues to circulate and to function normally until it is cleared by the spleen. The bacteria inside erythrocytes avoid the immune response, while also being accessible for transmission to a new host via a blood-feeding arthropod vector $[4,54]$. The signals that limit Bartonella replication inside erythrocytes, critical for the health of the host and the success of the organism, remain unknown, but are an intriguing area of future research. This attribute enables most Bartonella species to be stealth pathogens, frequently undetected in their host. The main exception to this is Bartonella bacilliformis, which lyses erythrocytes during acute Carrion's disease and is consequently potentially fatal.

The hypothesised infection strategy of $B$. henselae is illustrated in Figure 1.3 below. This figure depicts the transport of the bacteria to the bloodstream by immune cells and persistence inside endothelial cells, both points that remain the subject of debate. 


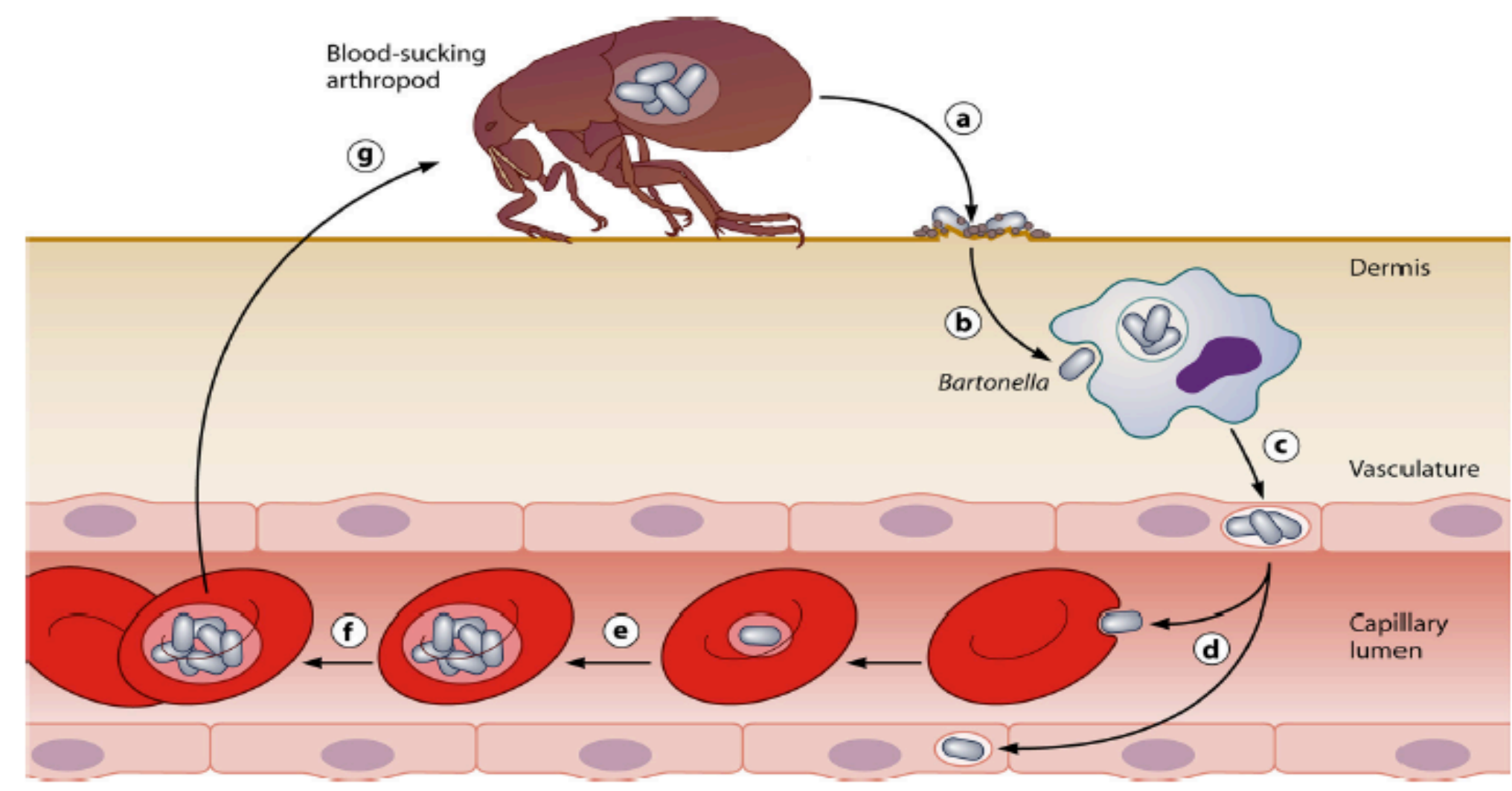

Figure 1.3: The hypothesised infection strategy of reservoir host infections by $\boldsymbol{B}$. henselae. The infected arthropod, shown here as a cat flea, takes a blood meal while simultaneously depositing Bartonella on the skin of the reservoir host. Upon scratching, the Bartonellae are taken up by resident immune cells, transported into the vascular endothelium (C), where the bacteria persist intracellularly without eliciting an immune response from the host. From the bloodseeding niche in the bloodstream, the bacteria periodically invade erythrocytes (D). After a limited number of replications, Bartonellae can persist inside the erythrocytes (E, F) and are competent for transmission by a new hematophagous arthropod (G). Reproduced with permission from The American Society for Microbiology. Harms \& Dehio (2012) [11]. 


\section{$1.4 \quad$ Evasion of innate immunity by Bartonella in mammalian hosts}

Bartonella species typically enter through the derma. The skin is an important defence against microbial attack, deploying both the innate and adaptive immune systems. Bacteria that penetrate the skin encounter resident dermal immune cells such as Langerhans cells, which detect bacterial antigens through Toll-like receptors, TLRs [55]. These TLRs recognise conserved microbial molecules, known as pathogen-associated molecular patterns (PAMPs), and initiate immune pathways. Microbes are often removed via phagocytosis by macrophages or neutrophils or killed via the complement system. However, Bartonella species have been reported to resist phagocytosis, allowing them to survive and enter the bloodstream. Little is known about their interaction with neutrophils or complement. There are contradictions within the literature surrounding the precise immune evasion mechanisms in different Bartonella spp. and this remains an area of active research.

Lipopolysaccharide (LPS) is a major PAMP of gram-negative bacteria, and a potent trigger for immune cells. The presence of LPS would usually result in TLR4 activation, cytokine production, B cell activation and antibody production; LPS in the bloodstream can also frequently lead to septic shock. However, this is rarely seen with bloodstream infections of Bartonella. The structure of B. quintana LPS, described by Malgorzata-Miller et al., may explain this observation [56]. Classical LPS models are characterised by a tripartite structure which consists of lipid A anchoring LPS to the outer membrane, a core oligosaccharide which works with lipid A to maintain the integrity of the outer membrane, and an $\mathrm{O}$ antigen polysaccharide connected to the core [56, 57]. In contrast, $B$. quintana LPS contains five fatty acid tails, a unique structure that not only is not recognised by TLR4 but is also a potent TLR4 antagonist [58]. B. quintana LPS blocked both the transcription and release of proinflammatory cytokines, TNF- $\alpha$, IL-1 $\beta$ and IL-6, from human monocytes stimulated with E. coli LPS, demonstrating an antagonistic effect on TLR4 [58]. The hypothesised model of B. quintana LPS is shown in Figure 1.4 below. 


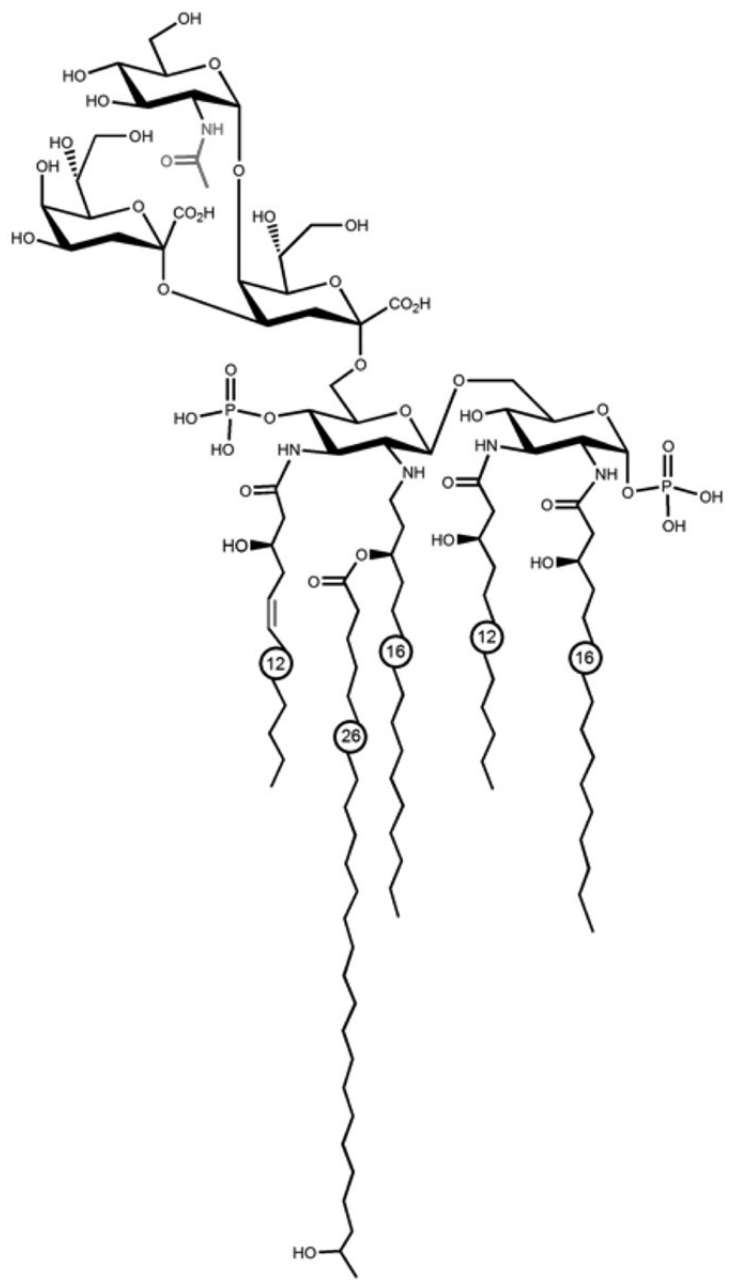

Figure 1.4 Hypothesised model of B. quintana LPS. The model of B. quintana LPS suggested from Malgorzata-Miller et al., 2016 is shown here [56]. Used freely under the Creative Commons CC BY License.

Adherence to host cells can also aid in avoiding clearance by the host through peristalsis, drainage through the lymphatic system or removal by innate immune cells. Adhesins, proteins expressed on the pathogen's surface, mediate bacterial adhesion to host molecules. These include the trimeric autotransporter adhesins (TAA) expressed on the surface of some gram-negative bacteria. The $B$. quintana TAAs known as VOMPs, are virulence factors required for mammalian infection [59]. The family of four VOMPs are immunodominant and variably expressed on the surface of $B$. quintana during prolonged bloodstream infection, in both natural human and experimental rhesus macaque infection [52]. The importance of VOMPs in adherence was shown through binding experiments where the interaction between $B$. quintana and primary endothelial cells was diminished in the absence of VOMPs [59]. B. quintana expresses four VOMPs, known as VompA-D and encoded by four 
genes (vompA-vompD) [59]. In B. quintana, the VOMPs confer two important virulence phenotypes: autoaggregation and collagen binding [59]. While the VOMPs are involved in bacterial adhesion to primary endothelial cells, they do not appear to be involved in bacterial adherence to epithelial e.g., HeLa-229, or phagocytic e.g., THP-1, cells [60]. The B. quintana VOMPs might have additional, unknown functions. For example, Bartonella escape phagocytosis and VOMPs may play a role in protecting against this immune response. Additionally, VOMPs may be involved in protecting Bartonella against complement, for example by binding to host factors such as vitronectin, a complement inhibitor. Together, these are important areas of potential future research.

B. quintana also expresses at least two Type IV Secretion Systems (T4SS). The T4SS is a specialised secretion system, found in many Gram-negative bacteria, that translocates macromolecules, including DNA or effector proteins, from the bacterial cytoplasm directly into a host cell $[61,62]$. The two B. quintana T4SS include the VirB/D4 system and the Trw system.

The VirB/D4 T4SS contributes to virulence by translocating Bartonella effector proteins, or BEPs, directly into host cells, subverting normal processes to facilitate infection [11]. Bacteria in the bloodstream are exposed to blood immune factors, including antibodies, complement and neutrophils; one of the ways Bartonella evade immune detection is by invading erythrocytes. Some Bartonella species, including B. quintana, achieve this through the Trw system, which mediates erythrocytic adhesion and invasion. The Trw is unusual for a T4SS, in that it evidently does not secrete any macromolecules into host cells; rather, the structure acts as an adhesin, enabling the bacteria to bind to and invade erythrocytes [11]. A Trw deletion mutant of Bartonella birtlesii, a mouse-specific Bartonella species, was unable to establish intra-erythrocytic infection in mice, demonstrating that the Trw system is necessary for the adherence to erythrocytes and does not translocate effectors [63]. The Trw system was further shown to mediate host specificity, by binding only to erythrocytes from the reservoir host [63].

There are likely many additional unknown Bartonella virulence factors which aid in resisting complement and phagocytosis that remain to be discovered. 


\subsection{Bartonella pathogenicity factors}

\subsubsection{T4SS - Evolution, structure and function}

Within the bacterial world, there are two main T4SS subfamilies: the conjugation machinery, which translocates single-stranded DNA substrates, and the effector translocators, which deliver protein or DNA substrates directly into eukaryotic cells [64]. Both subfamilies require direct cell-cell contact to function. While not all Bartonella spp. have a T4SS, these systems are critical to the infection process of the Bartonella species that do express them [64]. One of the best studied T4SS is the VirB/D4 system of Agrobacterium tumefaciens, which delivers bacterial DNA into plant cells. Many T4SS have been found in other Gram-negative bacteria and consist of homologues of most or all of the VirB/D4 subunits. This conservation in structure of the machine architecture reflects the common ancestry, however, there are prominent differences between T4SS in both composition and number of subunits [63]. While the T4SS in A. tumefaciens has been well-studied, the mechanism of substrate translocation remains incompletely understood.

Bartonella spp. can cause infections in incidental or reservoir hosts, but intraerythrocytic infection only occurs in the reservoir host. This specificity is mediated by the Trw system, one of the two one of two T4SS found in some Bartonella spp. [65]. The Trw locus was laterally acquired during evolution of the Bartonellae; it is only found in Group C of the major Bartonella lineages, and is thought to have been acquired through a single horizontal gene transfer event [10]. It is present in 13 species which are adapted to diverse mammalian hosts, but is absent from B. bacilliformis (human reservoir), Bartonella clarridgeiae (cat reservoir) and multiple species of Bartonella with ruminant reservoir hosts [63]. Interestingly, the acquisition of the Trw system correlates with loss of flagella, a major pathogenicity factor for $B$. bacilliformis in the invasion of erythrocytes [63]. The identification of the Trw system as a key virulence factor in reservoir host-specific adhesion has enabled researchers to identify its host cell target, band3, a major outer membrane glycoprotein of erythrocytes functioning in anion transport $[66,67]$. Band3 has been suggested as a possible erythrocyte receptor of $B$. bacilliformis and even to be involved in the malaria parasite invasion of erythrocytes $[66,68]$.

The VirB/D4 T4SS was first identified in Bartonella spp. 20 years ago, following the characterisation of the locus, which encodes a $17-\mathrm{kDa}$ immunodominant protein in $B$. henselae $[11,69,70]$. Similar to the prototypic VirB/D4 T4SS of $A$. tumefaciens, the B. quintana T4SS is composed of at least 10 components (VirB2-VirB11) with a strictly conserved gene order, and the downstream gene, VirD4 [71]. The VirB/D4 T4SS is only found in Group B and C 
Bartonella. Figure 1.5 below outlines two potential models of the VirB/D4 T4SS machinery for substrate translocation. 


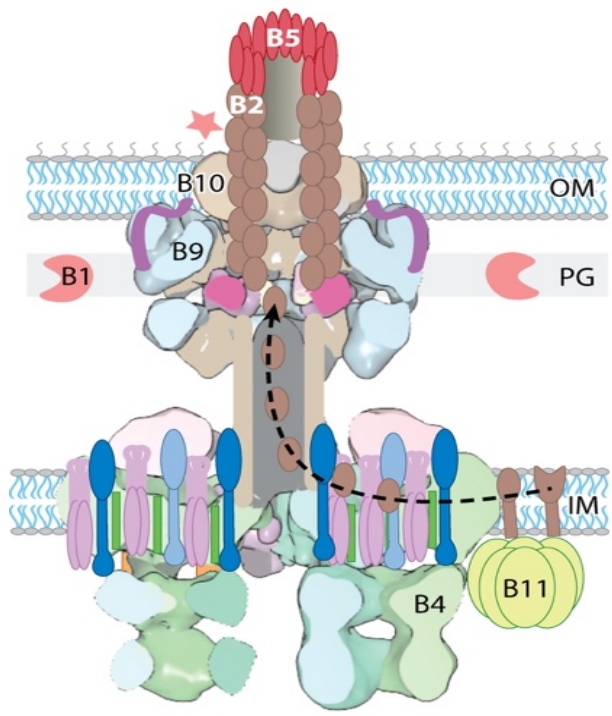

VirB1
VirB7 b

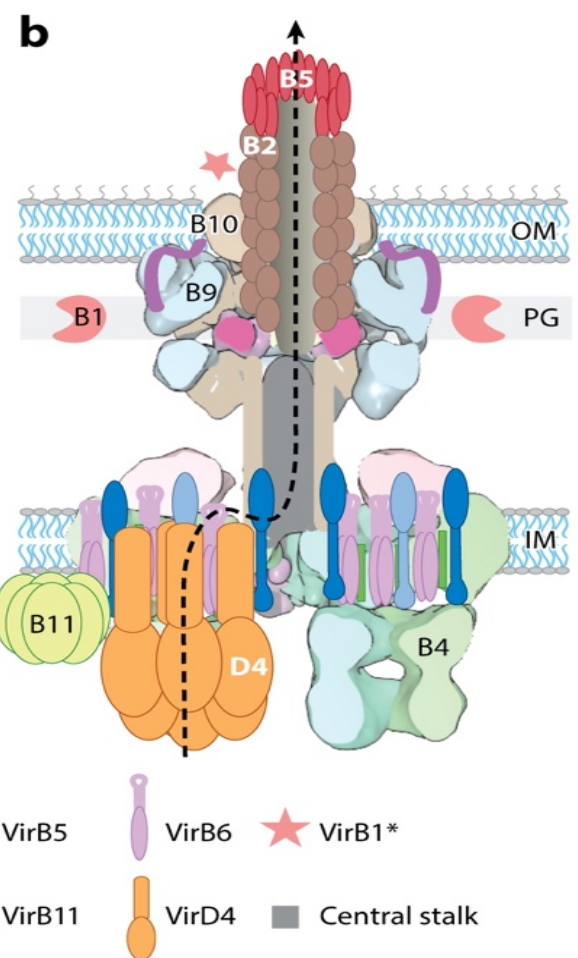

C

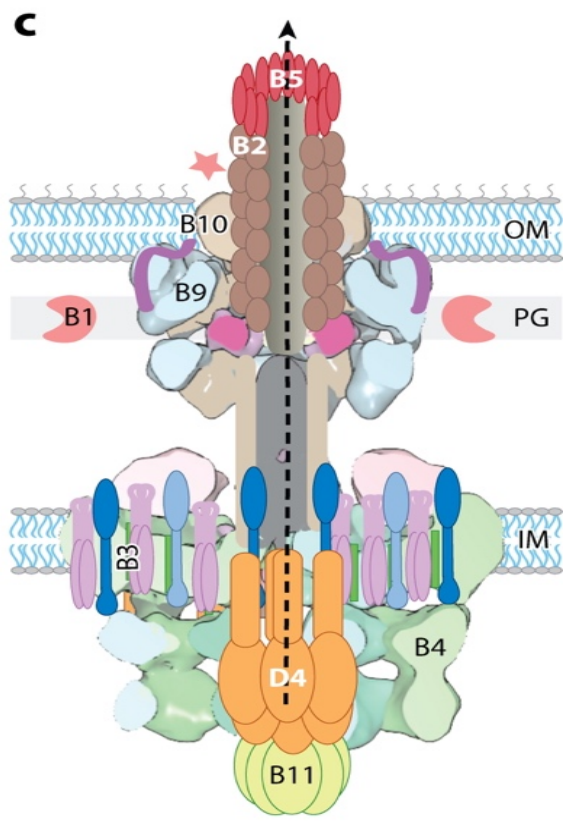

R Annu. Rev. Biochem. 84:603-29

Figure 1.5. Current models for the T4SS pilus biogenesis and substrate translocation. (a) Pilus biogenesis. (b) A one-step translocation pathway using the VirD4 coupling protein assisted by cytoplasmic ATPases. Panel b illustrates the possibility that one of the hexametric VirB4 barrels at the base, seen in green, could be substituted with the VirD4 hexamer, providing a channel continuous with the central stalk (grey) for substrate translocation. (c) A one-step translocation pathway utilising the VirD4 coupling protein. Panel c illustrates a second possibility wherein VirD4 is found next to the VirB4 hexametric barrels. The complex could switch to a secretion-competent form through VirB11 stacking next to or under VirD4, as seen in panel b. Chandran Darbari \& Waksman., 2015 [71]. Used with permission from Annual Reviews. 


\subsubsection{Bartonella gene transfer agents}

Bacteria in isolated populations are at risk of suffering from the accumulation of deleterious mutations, affecting fitness and survival and possibly leading to extinction of the population [72]. This evolutionary phenomenon is known as Muller's ratchet and is the typical fate of populations that experience frequent bottlenecks [72, 73]. Bartonella species, which are highly host-adapted pathogens with a complex infection cycle, represent such a population. One route to escape Muller's ratchet is through homologous recombination and horizontal gene transfer, HGT, processes which result in the repair of deleterious mutated genes or the acquisition of new genes that offset the effects of accumulating mutations [74].

A major source of HGT in bacteria are the recently discovered gene transfer agents, GTAs. GTAs are bacteriophage-like particles that promote exchange of genetic material between individual cells through the process of cell lysis and release of bacteriophage-like intermediates $[72,75,76]$. The best studied GTA is RcGTA, encoded in the genome of the alpha-proteobacterium Rhodobacter capsulatus. Several homologues of the RcGTA system have been discovered in other lineages of alpha-proteobacteria. A novel GTA was discovered in Bartonella and named BaGTA [76]. The Bartonella GTA is highly conserved and is present in the precise same genomic location in all Bartonella spp. $[10,72,76]$. The BaGTA has been proposed to drive the exchange and diversification of host-interaction factors within Bartonella communities, including the VirB/D4 T4SS and effector proteins. Furthermore, HGT mediated by BaGTA could influence host adaptability of Bartonella and may potentially result in the emergence of a novel pathogen [72].

The BaGTA cycle begins with GTA activation within fast-growing donor bacteria. Bacteria use various mechanisms to respond appropriately to changing environments and to optimize chances of survival. One of the major stress responses by all bacteria is dependent on the accumulation of a specific alarmone, ppGpp, a chemical messenger which allows a metabolic switch in bacteria from anabolic processes to a "survival mode", down-regulating growth, transcription, translation and the cell cycle [77, 78]. Next, assembly of the BaGTA occurs through cleavage and packaging of DNA, via unknown mechanisms. The GTAs are released via cell lysis, after which GTA absorption occurs on growing recipient bacteria. Incoming BaGTA DNA is imported into the recipient's cytoplasm, where it undergoes homologous recombination and is incorporated into the chromosome. The process is summarised in Figure 1.6 below. BaGTA-mediated HGT might represent a solution to countering Muller's ratchet but also allow the accumulation of beneficial traits. Various studies 
have attempted to elucidate the role of BaGTA in Bartonella pathogenesis, however findings are largely circumstantial and require further research $[79,80]$.

(c)

(c) GTA

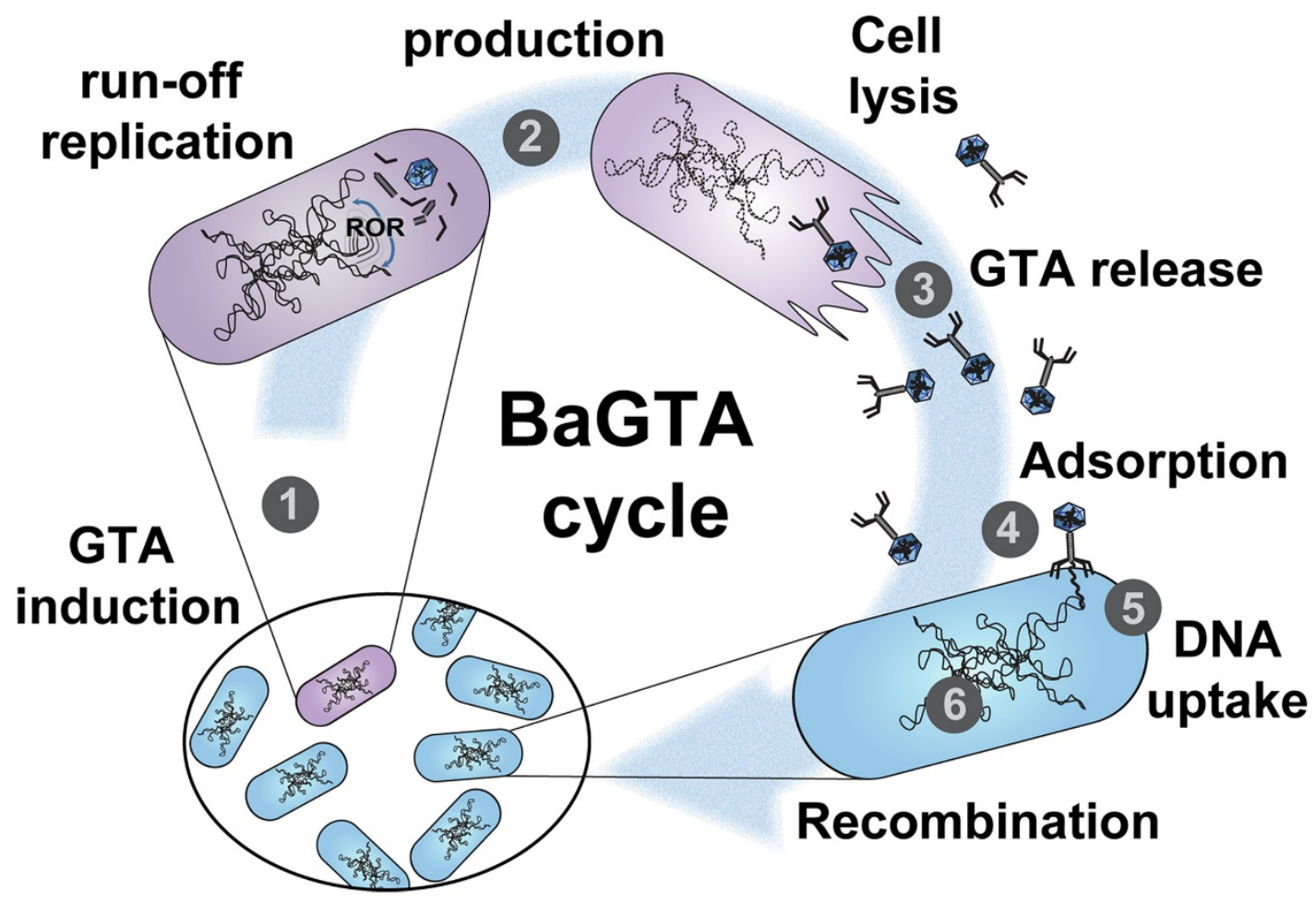

Figure 1.6. The BaGTA and DNA transfer cycle. The BaGTA cycle begins with GTA activation in the donor (1). The induction of GTAs is limited to fast-growing bacteria regulated through cellular ppGpp levels. (2) Cleaved and packaged DNA fragments of $\sim 14 \mathrm{~kb}$ are assembled into the BaGTAs, which are then ready for release via cell lysis (3). GTA adsorption (4) occurs on the recipient through GTA docking via an unknown process. The BaGTA delivers the DNA fragments into the recipient's cytoplasm (5) where homologous recombination incorporates the DNA into the recipient's chromosome (6). Used freely under the Creative Common Attribution License. Québatte et al., 2019 [72]. 


\subsubsection{Bartonella type 4 secreted effector protein structure and function}

The secreted Bartonella effector proteins, Beps, are encoded on a pathogenicity island that also encodes the VirB/D4 T4SS structural components in B. quintana and B. henselae, as shown in Figure 1.7. Most characterised Beps have a modular structure with an N-terminal domain that interacts with the host and a C-terminal bipartite secretion signal which is part of the Bartonella intracellular domain, or BID [82]. However, the notable exception is BepA1 and BepA2 of B. quintana, where the two modular structures are encoded by two adjacent open reading frames. The Bep genes are not fully conserved between Bartonella spp. as they display a high degree of plasticity. Several effector proteins in B. quintana and B. henselae contain an $\mathrm{N}$-terminal AMPylation (filamentation induced by $\underline{\mathrm{c}}$ AMP, or FIC) domain, which is followed by a short binding fold and the secretion signal [11]. The effector proteins of $B$. henselae are well characterised, in contrast to the B. quintana orthologues. B. quintana has six Bep proteins: BepA1, BepA2, Bep C, BepE, BepF1 and BepF2, while B. henselae has seven Bep proteins (BepA - G). Figure 1.7 shows the organisation of the VirB/D4/Bep pathogenicity islands in $B$. quintana, and $B$. henselae and, as a comparison, the phylogenetic relationship of these pathogenicity islands with the AvhB/Tra conjugation system in A. tumefaciens [83]. Several Bep proteins harbour more than one BID domain, raising the question as to whether these additional BID domains have evolved additional functions. Various studies have shown many Bep-induced cellular phenotypes associated with $B$. henselae BID domains, including altering small GTPase activity and adenylyl cyclase activation [84-86]. Bartonella effector protein G (BepG) of B. henselae was the first described Bep protein with a functional BID-domain and was shown to inhibit endocytic uptake of bacteria and induce invasome formation in endothelial cells [85]. Additionally, B. henselae BepF and $\mathrm{BepC}$ have been shown to induce invasome formation [86]. BepF has three BID domains and one FIC domain while BepC contains one BID domain and one FIC domain [87].

The first two BID domains of B. henselae BepF activate the small GTPase, Cdc42. Although the function and target/s of $B$. henselae BepC are unknown, it plays a role in bacterial uptake into host cells, as shown by an in vitro infection model [88]. 
B. quintana

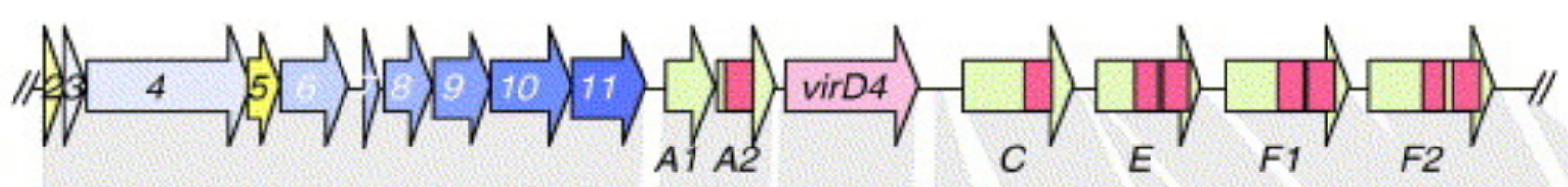

$\varnothing 86 \%$

$61 \%$

$91 \% \Delta \Delta$

\section{$73 \%$}

$\begin{array}{lllll}\Delta & 50 \% & 32 \% & 45 \% & \Delta\end{array}$

B. henselae

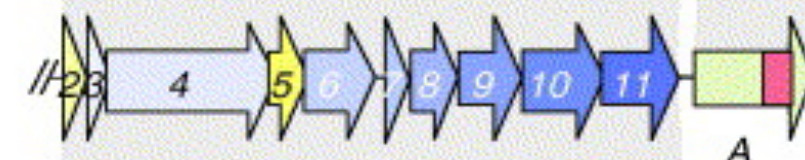

A
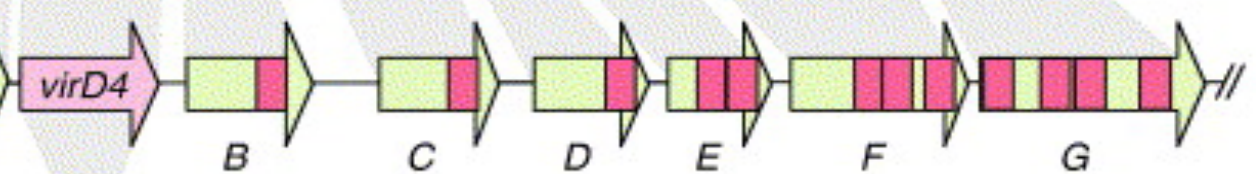

Ø $43 \%$

$52 \%$

A. tumefaciens

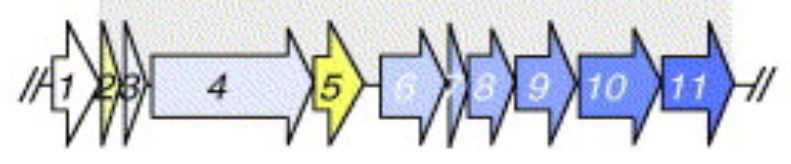

$a v h B$

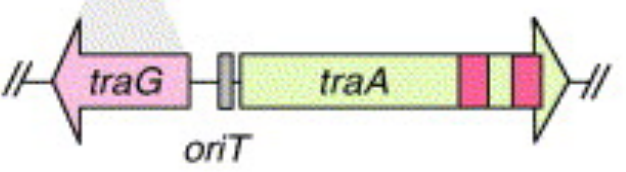

oriT

Figure 1.7 The virB/virD4/Bep pathogenicity island of $B$. henselae and B. quintana. The genetic organisations of the virB/virD4/Bep open reading frames are shown for B. quintana and B. henselae. The Bep proteins in both Bartonella spp. are represented by the green arrows. The VirB components are represented by the yellow and blue arrows, and the VirD4 protein is represented by the pink arrow. The BID domain in each Bep protein is represented by the darker pink rectangle within each Bep protein. The AvhB/Tra conjugation system in A. tumefaciens is included to demonstrate the phylogenetic relationship between each system. The degree of conservation for individual genes or groups of genes (on average, $\varnothing$ ) is indicated in percentages of amino acid identity of predicted proteins. Colour code: Yellow: pilus-associated extracellular components of the T4SS machinery. Schröder \& Dehio, 2005 [83]. Reproduced with permission from Elsevier. 
It has also been shown that $B$. henselae BepE protects cells from fragmentation effects triggered by other Beps and promotes dissemination from the dermal inoculation site to the bloodstream [89]. Recently Wang et al. reported that B. quintana BepE, when transfected into HeLa cells, induces filopodia and membrane ruffle formation [88]. BepE also undergoes polyubiquitination and activates the cellular autophagy response, a response only seen with the $B$. quintana BepE homologue [88]. Cellular autophagy is an innate immune pathway that targets intracellular bacteria by containing and killing the bacteria in an autolysosome. It may also be a host cell response to bacterial cytotoxins. Studies have demonstrated that intracellular bacteria have evolved strategies to combat autophagy machinery e.g. by coating themselves in host proteins to avoid autophagy recognition [90].

The $B$. henselae effector protein A (BepA) has been characterised as well and contains one BID domain and one FIC domain [87, 91]. In B. quintana, the domains are found in two adjacent open reading frames, termed BepA1 and BepA2. B. quintana BepA1 encodes a FIC domain, and BepA2 encodes a BID domain and positively charged C-terminal tail, the putative type 4 secretion signal. In B. henselae, the BepA BID domain has been shown to mediate BepA translocation into endothelial cells and to inhibit apoptosis by binding to host adenylyl cyclase to potentiate a $\mathrm{G} \alpha$ subunit-mediated increase in cAMP levels $[84,91,92]$. The B. quintana BepA2 protein is translocated into endothelial cells and, similar to B. henselae BepA, also confers anti-apoptotic activity [92]. B. quintana BepA1 encodes a FIC domain but lacks a putative type 4 secretion signal. Therefore, it is uncertain whether BepA1 is translocated into endothelial cells, or if it forms a complex with BepA2 for translocation; these are key areas for future research. Apoptosis is a potential defensive response when bacterial proteins, such as the Beps, are translocated into host cells. Yet both B. henselae and B. quintana cause the formation of vasoproliferative tumours in infected individuals, suggesting that the prevention of programmed cell death is a key aspect of Bartonella pathogenesis.

Bacteria inside endothelial cells are protected from antibody- and complementmediated immune responses, but infected endothelial cells can be killed by cytotoxic $\mathrm{T}$ lymphocyte (CTL) mediated apoptosis. Schmid et al demonstrated that BepA protects endothelial cells from MHC class I-restricted apoptotic activity of CTLs [92]. This study further showed that the inhibition of apoptosis is confined to the conserved BID domain, and that the FIC domain is dispensable for the inhibition of apoptosis. Thus, the roles of the FIC domains of $B$. henselae BepA or B. quintana BepA1 in host cell interactions remain unclear. 


\subsection{The FIC domain}

The conserved enzymatic FIC domain catalyses AMPylation, the covalent transfer of an AMP moiety onto hydroxyl side chains of a target protein [93]. The FIC domain was first discovered in 1969 in E. coli, where the adenylyl transferase enzyme AMPylates glutamine synthetase, regulating its activity [94, 95]. Only in the last decade have FIC proteins been implicated in bacterial pathogenesis. The role of the catalytic function of the FIC domain was shown by Yarbrough et al., and by Worby et al, with both groups demonstrating that bacterial FIC proteins covalently AMPylated the side chains of Rho GTPase signalling family members, typically on serine, threonine or tyrosine residues [96, 97]. Specifically, bacterial toxins with a FIC domain have been shown to interfere with Rho guanine triphosphate, GTPase family function and disrupt microtubule-dependent vesicular transport [98].

Furthermore, Rho GTPases are essential proteins for the proper functioning of cells as they regulate multiple processes e.g. cytoskeleton rearrangements, vesicular transport and progression of the cell cycle and cell motility to name a few [99]. AMPylation interferes with these functions, resulting in cytotoxicity. However, cellular toxicity within Bartonella infections is not as severe.

The bacterial effector VopS, of the food borne pathogen Vibrio parahaemolyticus, has a FIC domain and is delivered into the host cell via a type III secretion system [100]. Yarbrough et al., showed that when a Rac, a member of the Rho GTPase family, was coexpressed with VopS, the Rac protein mass increased by 329 daltons, consistent with the addition of an AMP moiety on the threonine 35 residue [96]. Substitution of the Thr35 with an alanine abolished the AMPylation activity of the VopS FIC domain [96]. AMPylation of Rac resulted in steric hinderance, preventing interactions between activated Rho GTPase proteins with signalling partners. FIC domain proteins, which AMPylate Rho GTPase family proteins and alter regulation of the actin cytoskeleton, are found in effector proteins and toxins from multiple bacterial pathogens, including Legionella pneumophila and Histophilus somni [97, $101]$.

FIC domains are defined by a core $\operatorname{HPExGN}(\mathrm{G} / \mathrm{K}) \mathrm{R}$ motif, with the conserved histidine shown to be essential for catalytic activity [93]. The FIC domain motif is found in B. henselae BepA, as well as BepC in B. henselae and B. quintana [102]. In B. henselae, the FIC domain sequence of BepB differs from the FIC motif in the last two amino acids (GR $\rightarrow \mathrm{EH}$ ), while $B$. quintana lacks a BepB homologue. 
One of the goals of studies of bacterial effectors with FIC domains is to identify the host cell proteins that are targeted and AMPylated, as this can provide insights into how host cells are manipulated. One approach involves incubating the FIC domain protein with cell lysates in the presence of ATP that has been labelled with radioactivity or a fluorophore, allowing the subsequent detection of the AMPylated protein $[96,97,103]$. The method with radioactive ATP has been used several times but requires special training and disposal of radioactive material. The use of a fluorophore-labelled ATP molecule has been proposed but has not been used to identify a target, and it is likely that the bulky fluorophore could interfere with the interaction $[84,103,104]$. Other approaches use advanced proteomics methods or antibodies that bind to AMPylated threonine or tyrosine [103, 105].

These methods have been used to identify the host cell target protein of one Bartonella BepA homologue. Peiles et al., used a mass-spectrometry based approach with stable isotopelabelled ATP to identify vimentin as the target of FIC-mediated AMPylation in B. rochalimaea [103]. Vimentin is a type III intermediate filament of mesenchymal derived cells that can interact with the RAF-1/RhoA signalling pathway, involved in mediating actin dynamics [106]. Self-AMPylation enzymatic activity has been detected with $B$. henselae BepA, but the host cell target remains unknown [102]. Despite their duplication and conservation, no Bartonella FIC domain effector has to date been demonstrated to contribute to virulence, either in vitro or in vivo [11].

\subsection{The BID domain}

The BID domain is an approximately 142-amino acid sequence with a positively charged C-terminal tail, which acts as a translocation signal for the Beps [47, 82]. Apart from B. quintana BepA1, each Bep contains at least one BID domain, with several Bartonella effector proteins harbouring multiple copies of the BID domains, e.g., BepE, BepF and BepG of $B$. henselae. This suggests additional BID domains, released from selective pressure to interact with the type 4 coupling protein, VirD4, may have evolved alternative, novel functions [107]. Indeed, many Bep-induced cellular phenotypes are linked to the BID domain with activity ranging from altered small GTPase function to adenylyl cyclase activation [84-86]. Regardless of the total number of BID domains, the one that is closest to the C-terminus enables translocation [82].

Structural analyses of three BID domains have shown a novel conserved fold, formed by a four-helix bundle topped with a hook [108]. The core of the BID domain is formed by 
conserved polar residues, but the surface has shown a high degree of variability even among orthologs, which supports the idea that these additional BID domains are able to adopt new functions [107].

\subsection{BepA1 preliminary data}

Bartonella are significantly understudied organisms, and little is understood about how they persist in their reservoir host. We hypothesize that Bartonella are able manipulate cytokine and chemokine signalling in their favour, but there is a lack of experimental data to support this.

B. quintana encodes six Bep proteins but little is known about these effectors and how they contribute to infection and persistence. BepA1 of B. quintana harbours a FIC domain, similar to those previously shown to be involved in pathogenesis of bacteria other than Bartonella. FIC domain proteins have been shown to catalyse AMPylation reactions of target host proteins, but in Bartonella this has only been shown for the BepA homologue of $B$. rochalimae. Therefore, to address the current gaps in understanding about B. quintana immune evasion and intracellular persistence we endeavoured to answer some of the outstanding questions about BepA1.

The FIC domain is likely important for $B$. quintana infection. The wide range of potential host cell target proteins results in many possible outcomes. Bartonella species are stealth pathogens and exhibit little to no cytotoxicity; therefore, it is unlikely that their effectors disable critical signalling proteins, such as the Rho GTPase family. The goal of this project was to use multiple approaches to investigate the effect of $B$. quintana BepA1 on host cells.

A former student in our lab carried out a yeast-two-hybrid system assay to identify potential protein-protein interactions between BepA1 Wild Type (WT) and a human cDNA library. Two independent hits were obtained against cDNA encoding a protein called Myozap, or myocardial zonula adherens protein, a 54-kDa conserved protein highly expressed in cardiac and lung tissue [109]. Myozap is a signalling protein that interacts with myosin phosphataseRhoA interacting protein (MRIP) to inhibit RhoA, a signalling protein that regulates the cytoskeleton and other cellular pathways [110]. Myozap also functions as an activator of Rhodependent serum response factor (SRF) signalling. RhoA GTPase regulates additional transcription factors, including NF-kB [111]. Previous work had also suggested that $B$. quintana BepA1 was suppressing the secretion of IL-6 and IL-8, cytokines that are regulated 
via the NF-кB pathway. However, these experiments did not show that AMPylation was required for cytokine suppression.

Based on these preliminary findings, as well as the nature of B. quintana as a stealth pathogen that subverts innate immunity, we asked whether BepA1 downregulates innate immune responses via AMPylation of target host proteins. Specifically, we asked if transfection of human cells with B. quintana BepA1, or a site-directed catalytically dead FIC mutant version, affected the secretion of proinflammatory cytokines, in response to TNF- $\alpha$, a potent proinflammatory cytokine and activator of the NF-KB/IKK pathway.

Much remains unknown about $B$. quintana infection, in particular how the pathogen modulates immune responses and persists inside the host. The study of B. quintana BepA1 attempted to answer a few of these questions.

\subsection{Research aims and hypothesis}

Based on published data, we hypothesised that B. quintana BepA1 is secreted into vascular endothelial cells, via the VirB/D4 T4SS, where it interferes with NF-kB signalling, blocking cytokine secretion and recruitment of immune cells to the site of infection. To test this hypothesis, I have three aims:

1.) Determine whether transient transfection of HeLa cells with wild type BepA1 or a site-directed, catalytically inactive BepA1 FIC domain mutant, followed by TNF- $\alpha$ stimulation, alters the expression of a panel of cytokines.

2.) Perform RT-qPCR to confirm altered cytokine and chemokine gene expression.

3.) Create an isogenic BepA1_BepA2 mutant by constructing a mutagenic plasmid and conjugating into $B$. quintana, followed by rounds of positive and negative selections.

This project focused on a broad characterisation of the innate immune response of HeLa cells with BepA1 WT (wild type) and FIC domain mutant transfections. Understanding how $B$. quintana is modulating immune responses opens doors to a better understanding of the early stages of Bartonella infection and to aid in development of better diagnostic methods and treatments. With this research, we hope to shed light on how B. quintana hampers immune signalling during infection, leading to intracellular persistence and survival in the host. 


\section{Chapter 2 Methods}

\subsection{Bacterial strains and growth conditions}

Bacterial strains used in this study, including their characteristics and source, are described in Table 2.1 E. coli was used as a host organism for the generation and maintenance of plasmids. E. coli strains were grown at $36^{\circ} \mathrm{C}$ overnight on LB agar plates or in LB broth with shaking, supplemented with antibiotics as needed. Glycerol stocks were made by combining an overnight culture 1:1 with cryopreservation medium ( $25 \%$ glycerol) in a cryovial and were stored at $-80^{\circ} \mathrm{C}$. B. quintana strain JK-31 glycerol stocks, made with M199S supplemented with $10 \%$ DMSO, were stored at $-80^{\circ} \mathrm{C}$. To revive $B$. quintana, the frozen culture was pierced with an inoculation loop and placed onto chocolate agar plates and streaked out. The inverted streaked plates were placed in a large glass jar with a lit candle and the jar lid sealed tight. The candle is extinguished due to the build-up of $\mathrm{CO}_{2}$. B. quintana was grown in a $36^{\circ} \mathrm{C}$ incubator, with colonies appearing after 8-10 days following revival from a freezer stock. Plates were subsequently passaged every 5-7 days, with fresh stocks revived after 5-6 passages.

\begin{tabular}{|c|c|c|}
\hline Strain & Characteristics & Source \\
\hline $\begin{array}{l}\text { Bartonella } \\
\text { quintana wild- } \\
\text { type strain JK-31 }\end{array}$ & $\begin{array}{l}\text { Human isolate (March 1993) from a patient with } \\
\text { bacillary angiomatosis in San Francisco. } \\
\text { Four gene VOMP locus intact. } \\
\text { Inherent resistance to nalidixic acid and cefazolin. }\end{array}$ & $\begin{array}{l}\text { Zhang et al., } 2004 \\
{[52]} \\
\text { Obtained from BEI } \\
\text { Resources, NIAID, } \\
\text { NIH-Bartonella } \\
\text { quintana Strain JK- } \\
\text { 31, NR-31832. }\end{array}$ \\
\hline E. coli $\mathrm{S} 17-1$ & $\begin{array}{l}\text { Mobilisation with chromosomally integrated tra } \\
\text { genes. } \\
\text { Genotype: TpR SmR recA, thi, pro, hsdR- } \\
\text { M+RP4: 2-Tc:Mu: Km Tn7 } \lambda \text { pir }\end{array}$ & $\begin{array}{l}\text { ATCC } 47055 \\
\text { A kind gift from } \\
\text { Dr. Jeremy Owen. }\end{array}$ \\
\hline $\begin{array}{l}\text { Lucigen 10G E. } \\
\text { cloni }\end{array}$ & $\begin{array}{l}\text { E. coli derivative optimised for transformation by } \\
\text { heat shock. } \\
\text { Genotype: F-mcrA } \Delta(\text { mrr-hsdRMS-mcrBC), } \\
\text { endA1, recA1, } \varphi 80 \mathrm{dlacZ} \Delta \mathrm{M} 15, \Delta \mathrm{lacX} 74 \text {, } \\
\text { araD139 } \Delta(\text { ara,leu }) 7697 \text {, galU, galK, rpsL (StrR), } \\
\text { nupG } \lambda \text { - tonA }\end{array}$ & Lucigen \\
\hline $\begin{array}{l}\text { NEB 10-beta } \\
\text { competent } E \text {. coli } \\
\text { (High Efficiency) }\end{array}$ & $\begin{array}{l}\text { E. coli cloning strain } \\
\text { Genotype: mcrAD (mrr-hsdRMS-mcrBC), endA1, } \\
\text { fhuA2, recA1 }\end{array}$ & $\begin{array}{l}\text { New England } \\
\text { Biolabs }\end{array}$ \\
\hline
\end{tabular}

Table 2.1 Bacterial strains used in this study with their respective characteristics and source. 


\subsection{Bacterial plasmids}

Bacterial plasmids that were used or constructed as part of this study are listed in

Table 2.2. Construction of plasmids is described in more detail below.

\begin{tabular}{|c|c|c|}
\hline Plasmid & Characteristics & Source \\
\hline pcDNA3.1 & $\begin{array}{l}\text { Mammalian expression vector; } \mathrm{Amp}^{\mathrm{R}}, \mathrm{SV} 40 \\
\text { origin, pUC ori for selection and } \\
\text { maintenance in E. coli. }\end{array}$ & $\begin{array}{l}\text { Life } \\
\text { Technologies }\end{array}$ \\
\hline pEX18Gm & $\begin{array}{l}\text { Suicide vector used for gene replacement; } \\
\mathrm{Gm}^{\mathrm{R}} \text {; } \text { ori } T^{+} s a c B^{+} \text {, with multiple cloning site } \\
\text { from pUC18. } \\
\text { Co1E1 ori. }\end{array}$ & [112] \\
\hline $\begin{array}{l}\text { pcDNA3.1_BepA1 FIC } \\
\text { mutant }\end{array}$ & $\begin{array}{l}\text { Encodes the BepA1 synthetic gene but with } \\
\text { point mutation that changes the conserved } \\
\text { FIC histidine to alanine (H171A) }\end{array}$ & This study \\
\hline $\begin{array}{l}\text { pEX18Gm_BepA1_BepA2 } \\
\text { mutagenesis plasmid }\end{array}$ & $\begin{array}{l}\text { Suicide vector used for BepA1 and BepA2 } \\
\text { gene replacement; } \mathrm{Gm}^{\mathrm{R}} \text {; ori } T^{+} s a c B^{+} \text {, } \\
\text { multiple cloning site from pUC18. Lacks the } \\
\text { BepA1 and BepA2 genes of } B \text {. quintana } \\
\text { wildtype JK-31 strain but includes the } \\
\text { immediate } \sim 800 \text { bp flanking regions of both } \\
\text { genes. }\end{array}$ & This study \\
\hline pcDNA3.1_BepA1 & $\begin{array}{l}\text { Encodes } B . \text { quintana wildtype JK-31 strain } \\
\text { BepA1 gene. }\end{array}$ & [113] \\
\hline pcDNA3.1_YopJ_GFP & $\begin{array}{l}\text { Encodes } B . \text { quintana wildtype JK-31 strain } \\
\text { YopJ gene, fused to a GFP using CT-GFP } \\
\text { Topo Fusion kit (Life Technologies). }\end{array}$ & [114] \\
\hline pSRKGm & $\begin{array}{l}\text { Mobilisable pBBR1MCS-5-derived broad- } \\
\text { host-range expression vector } \\
\text { containing lac promoter and } \operatorname{lacI}^{q}, \operatorname{lac} \mathrm{C}^{+} \text {, } \\
\text { and } \mathrm{Gm}^{\mathrm{R}} \text {. }\end{array}$ & $\begin{array}{l}\text { A kind gift } \\
\text { from Clay } \\
\text { Fuqua, } \\
\text { Indiana } \\
\text { University, } \\
\text { Bloomington. } \\
\text { [81] }\end{array}$ \\
\hline
\end{tabular}

Table 2.2 Bacterial plasmids used in this study with their characteristics. 


\subsection{Media recipes}

All bacterial media preparations, excluding M199S media, were autoclaved at $121^{\circ} \mathrm{C}$ for 15 minutes. Agar solutions were cooled to $50^{\circ} \mathrm{C}$ before antibiotics or vitamin supplements were added as required.

\section{Antibiotic supplementation}

Antibiotics were added to all autoclaved agar solutions and to liquid culture media as required. Antibiotics with final concentrations were added as follows: ampicillin $(100 \mu \mathrm{g} / \mathrm{mL})$, kanamycin $(50 \mu \mathrm{g} / \mathrm{mL})$, gentamicin $(25 \mu \mathrm{g} / \mathrm{mL}$ for LB agar plates, $12.5 \mu \mathrm{g} / \mathrm{mL}$ for liquid media, or $10 \mu \mathrm{g} / \mathrm{ml}$ for chocolate agar plates), chloramphenicol (35 $\mu \mathrm{g} / \mathrm{mL})$, vancomycin (5 $\mu \mathrm{g} / \mathrm{mL})$, nalidixic acid $(20 \mu \mathrm{g} / \mathrm{ml})$ and cefazolin $(2 \mu \mathrm{g} / \mathrm{ml})$.

\section{Liquid media}

Lysogeny broth (LB)

LB powder (Acumedia) was combined with double distilled water $\left(\mathrm{ddH}_{2} \mathrm{O}\right)$ to create a broth used for growing $E$. coli. LB broth was made by combining $20 \mathrm{~g}$ of Lennox powdered medium (Acumedia) with $1 \mathrm{~L}$ of $\mathrm{ddH}_{2} \mathrm{O}$, then autoclaved to sterilise.

\section{$\underline{\text { 2xYT Media }}$}

$2 x$ YT Media, a nutritionally rich broth for growing $E$. coli, was made by combining 31 g of 2xYT (Yeast Extract Tryptone) Medium powder (Difco) with $1 \mathrm{~L}$ of $\mathrm{ddH}_{2} \mathrm{O}$, agitating with heating to dissolve, and then autoclaving to sterilise.

\section{Cryopreservation media}

Cryopreservation media is used to sustain bacteria in culture when frozen. Cryopreservation media was made by combining $25 \% \mathrm{v} / \mathrm{v}$ glycerol (ThermoFisher Scientific) with $\mathrm{ddH}_{2} \mathrm{O}$ and autoclaving to sterilise.

\section{M199S media}

M199S media was prepared by combining 78 mL of Medium 199 with Earle's Balanced Salt Solution (HyClone, GE Life Technologies), $20 \mathrm{~mL}$ (final 20\%) fetal bovine calf serum, 1 mL 100X GlutaMAX (200mM stock solution concentration) and $1 \mathrm{~mL}$ Sodium Pyruvate $(100 \mathrm{mM})($ Gibco, Life Technologies). The medium was purified over a $0.22 \mu \mathrm{m}$ vacuum filter flask. 


\section{Solid media}

\section{LB Agar plates}

LB agar plates were poured by combining $16 \mathrm{~g}$ of LB agar powder (Invitrogen) with $500 \mathrm{~mL}$ of $\mathrm{ddH}_{2} \mathrm{O}$, mixing, autoclaving to sterilise, and pouring into petri dishes.

\section{Chocolate Agar plates}

Chocolate agar plates are a non-selective media supplemented with haemoglobin used for culturing B. quintana. These plates were prepared by combining $36 \mathrm{~g}$ of GC Agar powder

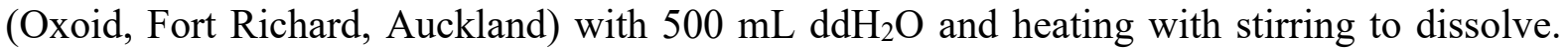
Upon reaching a boil, the media was autoclaved as previously described. Separately, $10 \mathrm{~g}$ of freeze-dried BBL Bovine Haemoglobin (Becton, Dickinson \& Company) was dissolved in a total of $500 \mathrm{~mL}$ of water, which was added in small increments. The solution was first mixed manually using a serological pipette, then placed on a stir plate without heat, until ready to autoclave.

After autoclaving, both flasks were placed in a $55^{\circ} \mathrm{C}$-water bath for 30 minutes. The flasks were then removed from the water bath, dried and sprayed thoroughly with $70 \%$ ethanol, and moved into a Class II biosafety cabinet. The haemoglobin solution was aseptically poured into the GC agar flask and contents mixed thoroughly by swirling. The combined flask was then placed back into a water bath set at $45^{\circ} \mathrm{C}$ for another 30 minutes. Following the second incubation, antibiotics and $10 \mathrm{ml}$ of reconstituted BD BBL IsoVitalex enrichment medium (Becton Dickinson, Fort Richard, Auckland) were added.

Non-selective plates were only supplemented with vancomycin as this suppresses the growth of a common laboratory contaminant. For the conjugation selective plates, nalidixic acid and cefazolin were added to inhibit the growth of the $E$. coli conjugative strain. Integration of the pEX18Gm plasmid was selected for by supplementing the medium with gentamicin.

The molten agar $(28 \mathrm{~mL} /$ plate) was dispensed into petri dishes using a serological pipette. All plates were allowed to air dry in the biosafety cabinet, upright with lids slightly askew, for 15 minutes before being sealed in plastic and stored at $4^{\circ} \mathrm{C}$. Plates were discarded if not used after one week. 


\subsection{Standard Molecular Biology Techniques}

$\underline{\text { Primers }}$

Primers used in this study were either designed using Primer3 software, selected from a primer list on the sequencing company website, or obtained from the literature [115]. All primers, listed in Tables 2.3 and 2.4, were ordered from Integrated DNA Technologies (Singapore) and supplied lyophilised. For long term storage, primers were suspended in sterile $\mathrm{ddH}_{2} \mathrm{O}$ to a final concentration of $100 \mu \mathrm{M}$. For routine working stocks, $10 \mu \mathrm{M}$ dilutions were made. All primers were stored at $-20^{\circ} \mathrm{C}$.

\begin{tabular}{|c|c|l|}
\hline $\begin{array}{c}\text { Primer } \\
\text { names }\end{array}$ & \multicolumn{1}{|c|}{ Primers sequence } & \multicolumn{1}{|c|}{ Features } \\
\hline BepA1_1 & 5'-GCGAGATCTAGCTGTAAAATGCCAAAAA-3' & $\begin{array}{l}\text { BglII } \\
\text { restriction } \\
\text { site } \\
\text { underlined }\end{array}$ \\
\hline BepA1_2 & 5'-GCCTCTCTGGCTGCTTTTGGCATAACGACCTC-3' & $\begin{array}{l}\text { Underlined } \\
\text { overlaps with } \\
\text { BepA1_3 }\end{array}$ \\
\hline BepA1_3 & 5'- ATGCCAAAAGCAGCCAGAGAGGCACAGAAACAA -3' & $\begin{array}{l}\text { Underlined } \\
\text { overlaps with } \\
\text { BepA1_2 }\end{array}$ \\
\hline BepA1_4 & 5'-GCGAGATCTACACGGTATCTTTCGCCAAC-3' & $\begin{array}{l}\text { BglII } \\
\text { restriction } \\
\text { site } \\
\text { underlined }\end{array}$ \\
\hline
\end{tabular}

Table 2.3 Primers used for generating the BepA1 mutagenesis plasmid.

\begin{tabular}{|c|c|c|}
\hline Primer names & Primers sequence & Source \\
\hline cyclA Forward & 5'- CTCATTGGAATTTTGCCGATT-3' & \multirow{2}{*}{$\begin{array}{l}\text { Kind gift from } \\
\text { Anne La } \\
\text { Flamme lab } \\
\text { group }\end{array}$} \\
\hline cyclA Reverse & 5'- CCCAGTGAAGATCCCCTTTTTA -3' & \\
\hline SM22 Forward & 5'- AGCCAGGAAGGGCCTGAGAAC -3' & \multirow[b]{2}{*}[116]{} \\
\hline SM22 Reverse & 5'- TGCCCAAAGCCATTAGAGTCCTC -3' & \\
\hline Myozap Forward & 5'- CAGGTGCTCTTTCCTCTTGG -3' & \multirow[b]{2}{*}{ This study } \\
\hline Myozap Reverse & 5'- AAGGGACTAGCGCATCAGAA -3' & \\
\hline IL-6 Forward & 5' - AGTGAGGAACAAGCCAGAGC -3' & \multirow{4}{*}[117]{} \\
\hline IL-6 Reverse & 5'- GTCAGGGGTGGTTATTGCAT -3' & \\
\hline IL-8 Forward & 5'- TCCTGATTTCTGCAGCTCTGT -3' & \\
\hline IL-8 Reverse & 5'-AAATTTGGGGTGGAAAGGTT -3' & \\
\hline
\end{tabular}

Table 2.4 Primers used in this study for RT-PCR and RT-qPCR. 


\section{$\underline{\text { Routine PCR }}$}

Conditions were optimised for specialised PCR experiments, e.g., RT-PCR. For cloning, the PCR parameters shown in Table 2.5 were used.

\begin{tabular}{|c|c|c|}
\hline Step & \multicolumn{2}{|c|}{ Cycle parameters } \\
\hline Initial denaturation & $95^{\circ} \mathrm{C}$ for $1 \mathrm{~min}$ \\
\hline Denaturation & $95^{\circ} \mathrm{C}$ for $30 \mathrm{sec}$ & \multirow{2}{*}{$\mathbf{3 0}$ cycles } \\
\hline Annealing/Extension & $68^{\circ} \mathrm{C}$ for $1-3 \mathrm{mins}$ & \\
\hline Extension & $68^{\circ} \mathrm{C}$ for $5 \mathrm{~min}$ & \\
\hline Hold & \multicolumn{2}{|c|}{$4^{\circ} \mathrm{C}$} \\
\hline
\end{tabular}

Table 2.5 PCR conditions used for generating the preliminary BepA1 and BepA2 PCR products for the mutagenesis plasmid, and the BepA1_2 mutagenesis PCR product. Extension for the $\sim 800$ bp preliminary PCR products was performed for one minute, while a three-minute extension step was used for the $1.6 \mathrm{~kb}$ fusion product.

\section{DNA manipulation and purification}

Restriction enzymes were obtained from New England Biolabs (Genesearch). Calf intestinal alkaline phosphatase (cIAP) and T4 DNA ligase were ordered from Life Technologies. Plasmid purifications and gel extractions were carried out with the Monarch Plasmid Miniprep Kit or the Monarch DNA Gel Extraction kit (New England Biolabs). Where needed, samples were purified using the Zymo DNA Clean and Concentrator-5 kit (Zymo Research, Ngaio Diagnostics).

Appropriate controls were included for the restriction digests and ligation reactions, including undigested vectors and reactions without ligase. For ligations, the insert:vector molar ratio was about $3: 1$.

\section{Transformation of E. coli}

Plasmid DNA or ligation reaction mixes were transformed by heat-shock into E. cloni ${ }^{\circledR}$ 10G chemically competent cells (Lucigen) according to the manufacturer's protocol. Briefly, the DNA or ligation reaction mix was combined with the thawed cells on ice in a $15-\mathrm{ml}$ polypropylene culture tube. The cells were shocked by heating to $42^{\circ} \mathrm{C}$ for 45 seconds in a water bath, then rescued with $1 \mathrm{ml}$ Recovery Medium (Lucigen) and incubated with shaking at $37^{\circ} \mathrm{C}$ for one hour. Transformants were recovered by plating on selective plates.

Transformations by electroporation were carried out using NEB 10-beta high efficiency competent cells (New England Biolabs). Prior to electroporation, 2-5 $\mu 1$ of plasmid DNA or ligation reaction mix were dialysed for 15-30 minutes on nitrocellulose filters over sterile 
distilled water. The dialysed DNA was then combined with the thawed electrocompetent cells in $0.2 \mathrm{~cm}$-gap electroporation cuvettes (Bio-Rad). A voltage of $2.5-\mathrm{kV}$ was applied to the bacteria, which were subsequently rescued by the addition of $1 \mathrm{ml}$ of Stable Outgrowth Medium (New England Biolabs) and incubation with shaking at $37^{\circ} \mathrm{C}$ for 1 hour. Transformants were selected by plating the bacteria on selective plates.

\section{DNA TAE agarose gels}

TAE agarose gels were used to analyse DNA samples. The gels were made by dissolving 1\% w/v agarose (Hydracare LE Multi-purpose Agarose) in 1X Tris-acetate EDTA buffer (made from 50X TAE buffer stock solution [ThermoFisher Scientific]). The agar suspension $(60 \mathrm{ml})$ was microwaved until boiling, then supplemented with $3 \mu \mathrm{L} 20,000 \mathrm{X}$ RedSafe nucleic acid stain (iNtRon, Ngaio Diagnostics). A 6X DNA loading dye (ThermoFisher Scientific) was added to samples, which were run alongside a $1 \mathrm{~kb}$ Plus Hyperladder DNA ladder (Bioline, Total Lab Systems) as a DNA size standard. Gels were run in $1 \mathrm{X}$ TAE running buffer at $100 \mathrm{~V}$ for 60 minutes or $85 \mathrm{~V}$ for 120 minutes. DNA was visualised using the Typhoon Imager (GE Healthcare Life Sciences) or under a UV light imager.

\section{Nucleic acid quantification}

DNA and RNA samples were quantified using the NanoDrop1000 spectrophotometer (ThermoFisher Scientific) with the appropriate sample setting. The NanoDrop was first cleaned with $\mathrm{ddH}_{2} \mathrm{O}$, blanked with the buffer the sample was eluted in, and the absorbance of the sample was then measured.

\section{Sequencing}

All sequencing reactions were performed by Macrogen Inc. (Seoul, South Korea) with standard primers appropriately selected from the website. 


\subsection{Construction of the BepA1 FIC mutant mammalian expression vector}

To assess the role of the FIC domain in B. quintana-host cell interactions with host cells, a mutant version of BepA1 was constructed and cloned into pcDNA3.1, a mammalian expression vector (Life Technologies). The conserved histidine residue of the FIC domain has been shown to be essential for catalytic activity, which was abrogated by replacing the histidine residue with an alanine [96].

The wild type BepA1 gene had previously been amplified by PCR and cloned into NheI and BamHI sites of pcDNA3.1, generating the BepA1 wild type expression vector [113]. Two base pairs of the codon for histidine 171 (CAC) were changed, resulting in one encoding alanine (GCG). The mutant BepA1 gene was synthesized as a double-stranded DNA fragment by Integrated DNA Technologies (Singapore). An NheI restriction site was engineered onto the 5' end, followed by a Kozak sequence, to aid in expression in mammalian cells, and the ATG start site of BepA1. A BamHI restriction site was engineered to the 3' end, immediately following the stop codon. The synthetic DNA product (500 ng) was supplied lyophilised and was resuspended in TE buffer to a final concentration of $10 \mathrm{ng} / \mu 1$. The suspension was vortexed and incubated at $50^{\circ} \mathrm{C}$ to fully resuspend the DNA. Both the insert and the pcDNA3.1 vector were digested with NdeI and BamHI overnight. The digested products were run out on an agarose gel and the bands purified from the gel. The purified digested DNA fragments were ligated with T4 DNA ligase overnight at $16^{\circ} \mathrm{C}$, then transformed into E. coli and selected on ampicillin plates. Successful cloning of the gene fragment was confirmed by restriction digest and sequencing of the plasmid. Please refer to Appendix figures 7.1 and 7.2 for sequence information about the BepA1 FIC domain mutant protein.

\subsection{Construction of the BepA1_BepA2 mutagenic plasmid}

To generate a B. quintana JK-31 markerless deletion strain, lacking both BepA1 and BepA2, we constructed a mutagenic plasmid with sequence flanking the targeted genes. This flanking sequence, sufficient to enable homologous recombination, was cloned into a vector, pEX18Gm, that contains genes for positive selection (i.e., gentamicin resistance) and negative selection (i.e., $s a c B$ to generate sucrose sensitivity [112]). The pEX18Gm plasmid also includes a lac $\alpha$ fragment adjacent to the multiple cloning site, to enable blue-white screening during cloning. Previous publications describing mutagenesis in Bartonella have suggested that 800$1000 \mathrm{bp}$ of homologous sequence is necessary for recombination to occur $[59,118]$. 
The flanking regions of B. quintana JK-31 BepA1 and BepA2 were first amplified by PCR using primers listed in Table 2.3. Primers BepA1_1 and BepA1_2 together amplified a product of $853 \mathrm{bp}$, while primers BepA1_3 and BepA1_4 yielded a product of $805 \mathrm{bp}$. The two resulting PCR products were checked on a gel, purified, and combined into a PCR fusion reaction using primers BepA1_1 and BepA1_4. Because the primers BepA1_2 and BepA1_3 were designed to overlap, the two PCR products self-primed to yield a fusion product of approximately 1,600 bp. For all reactions, the Advantage 2 PCR Kit (Takara Bio) was used. Primers BepA1_1 and BepA1_4 both have engineered BglII restriction sites, which generate compatible cohesive ends with BamHI. There was a BamHI sit in one of the amplified sequences, but neither were predicted to have a BglII site, which is why that enzyme was used.

The resulting fusion PCR product was confirmed to be the right size on a gel, purified, and digested overnight with BgIII. Purified pEX18Gm was digested overnight with BamHI, then treated with cIAP to prevent religation. The overnight digested products were run out on an agarose gel. DNA bands of the correct sizes were excised from the gel and the DNA extracted. The resulting purified, digested DNA was combined in a ligation reaction with T4 DNA ligase (ThermoFisher Scientific) and incubated overnight at $16^{\circ} \mathrm{C}$. Two plasmid-only controls were included, one that included ligase and one that did not. Following inactivation of the ligase, the reactions were transformed into E. coli and plated on LB plates with gentamicin, and IPTG and X-gal for blue-white screening. White colonies were cultured, and the plasmid checked by restriction digest and sequencing. After confirmation, the purified mutagenic plasmid was introduced into S17-1 E. coli cells by electroporation, as this conjugation strain can mobilise the plasmid into B. quintana.

\subsection{Cell Culture and Transfection}

\section{$\underline{\text { HeLa } 229 \text { cell cultures }}$}

To prepare cell culture flasks of HeLa 229 cells, frozen vials of cells were removed from liquid nitrogen storage and immediately placed in a $37^{\circ} \mathrm{C}$-water bath to thaw completely. The contents of the vial were slowly and aseptically added to a $15 \mathrm{~mL}$ Falcon tube containing 9 mL pre-warmed RPMI Medium 1640 (Gibco, Life Technologies), supplemented with 10\% foetal calf serum (FCS). The cells were pelleted by centrifugation at $300 \mathrm{x} g$ for 4 minutes. The supernatant was carefully decanted, and the cell pellet resuspended in $5 \mathrm{~mL}$ of warm $10 \%$ FCS RPMI medium and seeded into a T75 (Biofil, Interlab) tissue culture flask. The first passage 
from frozen stock was designated passage 1. Cells were passaged once per week, and after 5 total passages, new cultures were started.

For routine passaging, HeLa 229 cells were grown to $75-80 \%$ confluency, as determined by examining in an inverted microscope. Cells were gently washed with $5 \mathrm{~mL}$ of warm Phosphate Buffered Saline (PBS) buffer, then treated with $1.8 \mathrm{~mL}$ of warm trypsinEDTA solution (Gibco, TrypLE Express Enzyme, Life Technologies). After 3-5 minutes, 8 mL of $10 \%$ FCS RPMI medium was added and the suspension transferred to a $15 \mathrm{~mL}$ Falcon tube for centrifugation at $300 \mathrm{xg}$ for 4 minutes. The supernatant was then carefully decanted, and the cells resuspended in $5 \mathrm{~mL}$ warm RPMI medium. Of this suspension, $0.5 \mathrm{~mL}-1 \mathrm{~mL}$ was added to a T75 tissue culture flask containing $15 \mathrm{~mL}$ of warm RPMI medium. The flask was then placed in a $37^{\circ} \mathrm{C}, 5 \% \mathrm{CO}_{2}$ incubator.

For transfections, cells were washed as usual and then split into three T75 tissue culture flasks. Upon reaching $\sim 75 \%$ confluency, HeLa 229 cells were transferred to 6-well plate (Biofil, Interlab) at $6 \times 10^{5}$ cells per well in $2 \mathrm{~mL}$ of $10 \%$ FCS RPMI medium. Cells were incubated until 75-80\% confluent (about 1-2 days) and transfections performed.

\section{$\underline{\text { Transfections }}$}

For transfections, plasmids were purified from E. coli using the Monarch Miniprep Kit according to the manufacturer's instructions. Purified samples were pooled, quantified, then further concentrated using the Zymo Clean \& Concentrator kit. We used $2.5 \mu \mathrm{g}$ of plasmid per transfection, calculating the appropriate volume from the plasmid preparation.

The BepA1 wild type and BepA1 FIC mutant pcDNA3.1 plasmids were transfected in triplicate. Untransfected controls were included. For all transfections, a positive control plasmid, pcDNA3.1_YopJ_GFP, was included because the GFP fusion of this plasmid enabled the transfection to be evaluated by fluorescence microscope.

Transfections were performed using the Lipofectamine 3000 reagent (Life Technologies) and were optimised for $1.2 \times 10^{6}$ cells per transfection, or about $75-80 \%$ confluency in one well of a 6-well plate. To prepare plasmid DNA-lipid complexes, three tubes were labelled 'A', 'B' and 'C'. In tube A, the appropriate plasmid $(2.5 \mu \mathrm{g})$ was combined with $5 \mu \mathrm{L}$ of P3000 Enhancer reagent and brought to a final volume of $322.5 \mu \mathrm{L}$ with Opti-MEM medium (Life Technologies). In tube B, $322.5 \mu \mathrm{L}$ of Opti-MEM medium and $7.68 \mu \mathrm{L}$ Lipofectamine reagent were combined. The transfection cocktail was made by combining 300 $\mu \mathrm{L}$ from each tube ( $\mathrm{A}$ and $\mathrm{B}$ ) into tube $\mathrm{C}$ and incubating at room temperature for 10 minutes. If cells were seeded the day prior, the RPMI media was removed with a Pasteur pipette and 
replaced. The transfection cocktail from tube $\mathrm{C}(530 \mu \mathrm{L})$ was added dropwise, slowly and smoothly, to cells in one well. The cells were incubated for 48 hours at $37^{\circ} \mathrm{C}$, with another RPMI media change 24 hours after the transfections. Cells were treated with trypsin as described above and split into separate wells if the cells appeared to be overcrowded.

\section{$\underline{\text { TNF- } \alpha \text { stimulation }}$}

Following transfection, cells were incubated for 48 hours to allow them to recover before TNF- $\alpha$ stimulation. Recombinant TNF- $\alpha$ (Abcam) was made up in $\mathrm{ddH}_{2} \mathrm{O}$ at $100 \mathrm{ng} / \mu \mathrm{L}$ and stored at $-80^{\circ} \mathrm{C}$. A TNF- $\alpha$ solution was prepared with pre-warmed RPMI media such that each well of transfected cells (in a 6-well plate), received 2 ng of TNF- $\alpha$. TNF- $\alpha$ was mixed with RPMI media in a master mix before adding $2 \mathrm{~mL}$ of this solution to each well. All HeLa229 cells that received TNF- $\alpha$ stimulation were incubated for 1 hour at $37^{\circ} \mathrm{C}$.

\section{Cell medium sample preparation}

Following TNF- $\alpha$ stimulation, the cell culture medium was collected from each well, including from non-transfected and non-stimulated cells, and transferred to a microcentrifuge tube. The cell medium was centrifuged for one minute at $16,000 \times \mathrm{rpm}$ to remove cellular debris. The supernatant was transferred to a new microcentrifuge tube and stored at $-80^{\circ} \mathrm{C}$ until required.

\section{Cell lysate preparation for Human Cytokine Array Kit}

To prepare cell lysates, the cell medium was removed following 1 hour of TNF- $\alpha$ stimulation and cells were washed once with $1 \mathrm{ml}$ pre-warmed PBS. Protease inhibitors aprotinin (Sigma-Aldrich), leupeptin and pepstatin (both TOCRIS, In Vitro Technologies) were prepared in $1 \mathrm{mg} / \mathrm{ml}$ solutions in $\mathrm{ddH}_{2} \mathrm{O}$ (aprotinin and leupeptin) or ethanol (pepstatin) with gentle warming in a $45^{\circ} \mathrm{C}$-water bath. Each protease $(100 \mu \mathrm{l})$ was added to $1 \mathrm{~mL}$ Lysis Buffer 17 (R\&D Systems, In Vitro Technology). Cells were solubilised in Lysis Buffer 17 (1 $\mathrm{ml}$ per well), with pipetting to resuspend and lyse the cells. The cell lysates were rocked gently at $4^{\circ} \mathrm{C}$ for 30 minutes, then transferred to microcentrifuge tubes and centrifuged at $14,000 \mathrm{x} g$ for 5 minutes. The supernatant was transferred to a clean microcentrifuge tube and stored at $80^{\circ} \mathrm{C}$ until required. 


\section{Cell lysate sample preparation for RNA purification}

To purify mammalian RNA, the cell medium was removed following 1 hour of TNF- $\alpha$ stimulation, cells were washed once with $1 \mathrm{~mL}$ pre-warmed PBS, then $300 \mu \mathrm{L}$ of TRI Reagent Solution (ThermoFisher Scientific) was added to each well in a 6-well plate. The cells were lysed by pipetting the TRI Reagent, then the suspension from each well was transferred to microcentrifuge tubes, with triplicate wells pooled together into one tube. Samples were stored at $-80^{\circ} \mathrm{C}$ until required.

\section{Mammalian genomic DNA purification}

HeLa cells were grown to $75-80 \%$ confluency in one T75 tissue culture flask (Biofil, Interlab). The supernatant was carefully decanted, and cells were washed once with prewarmed PBS. Genomic DNA was purified using the Qiagen Gentra Puregene Yeast/Bact. kit (BioStrategy) according to the manufacturer's instructions for DNA purification from cultured cells, quantified via NanoDrop spectrophotometer, and stored at $-20^{\circ} \mathrm{C}$ until needed.

\subsection{Gene expression analysis}

\section{$\underline{\text { RNA Purification }}$}

All RNA handling was carried out in a dedicated fume hood. Surfaces were cleaned and wiped with $70 \%$ ethanol, followed by $0.5 \%$ SDS and $3 \%$ hydrogen peroxide. All instruments and equipment were sprayed with $70 \%$ ethanol before use.

Total RNA was purified from HeLa cells that had been stimulated with TNF- $\alpha$ and lysed with TRI Reagent Solution, as described above. The frozen tubes with pooled TRI Reagent lysates were thawed, combined with an equivalent volume of ethanol, and applied to a Direct-zol ${ }^{\mathrm{TM}}$ RNA Miniprep Kit (Zymo Research, Ngaio Diagnostics) column. The samples were centrifuged and washed according to the manufacturer's instructions. Additionally, an on-column DNase I treatment was done to remove carry-over host genomic DNA. RNA was eluted in $30 \mu \mathrm{L}$ of $\mathrm{DNase} / \mathrm{RNa}$ e free water and replicates were pooled into one RNase-free microfuge tube. RNA was quantified by NanoDrop spectrophotomer, and purity assessed by evaluating the A260/A280 and A260/A230 ratios.

RNA quality was assessed by running the samples on native agarose gels $(2 \% \mathrm{w} / \mathrm{v}$ agarose in $60 \mathrm{ml} 1 \mathrm{X}$ TAE buffer), supplemented with $5 \mu \mathrm{L}$ 20,000X RedSafe nucleic acid staining solution (iNtRon, Ngaio Diagnostics). A single stranded RNA ladder lower range (NEB, Genesearch) was used as a size standard and prepared according to manufacturer's 
instructions. Samples were combined with 2X RNA loading dye (NEB, Genesearch) as per manufacturer's instructions. Gels were run in $1 \mathrm{X}$ TAE running buffer for 1 hour at $120 \mathrm{~V}$ at $4^{\circ} \mathrm{C}$. RNA was visualised under a UV imager.

\section{cDNA Synthesis}

cDNA was synthesised using the iScript Advanced cDNA Synthesis Kit for RT-qPCR (Bio-Rad) according to the manufacturer's instructions. This kit uses a blend of oligo (dT) and random primers to amplify a range of target genes. Briefly, $1 \mu \mathrm{g}$ of RNA for each sample was combined, on ice, with the 5X iScript Advanced Reaction Mix and water, with the reverse transcriptase added last. For each sample, a control was set up without any reverse transcriptase added. The reactions were incubated at $46^{\circ} \mathrm{C}$ for 20 minutes, followed by one minute at $95^{\circ} \mathrm{C}$ to inactivate the reverse transcriptase.

cDNA was quantified via NanoDrop spectrophotometer using the single stranded DNA setting. Each cDNA sample ( $5 \mu \mathrm{l})$ was run on a 1\% TAE agarose gel and the gel analysed with the Amersham Imager 600 . cDNA was stored at $-20^{\circ} \mathrm{C}$ until needed.

\section{PCR Amplification of cDNA}

PCR was carried out by amplifying specific genes from total cDNA samples, using 1 $\mu \mathrm{g}$ of cDNA per sample and per reaction. The Taq PCR kit was used according to the instructions provided, with a total reaction volume of $50 \mu \mathrm{L}$ for all reactions. Kyratec PCR Thermocyclers (ThermoFisher Scientific) were used. The optimised annealing temperatures are summarised in Table 2.6 for each target gene. For each gene, the annealing and extension steps were done for 30 seconds.

\begin{tabular}{|c|c|c|}
\hline Target & Optimised annealing temp & Optimised annealing/extension time \\
\hline cyclA & $57^{\circ} \mathrm{C}$ & \\
\hline SM22 & $55^{\circ} \mathrm{C}$ & \multirow{2}{*}{$30 \mathrm{sec}$} \\
\hline IL-6 & $55^{\circ} \mathrm{C}$ & \\
\hline IL-8 & $52^{\circ} \mathrm{C}$ & \\
\hline Myozap & $56^{\circ} \mathrm{C}$ & \\
\hline
\end{tabular}

Table 2.6 Optimised annealing temperature for RT-PCR.

Afterwards, the PCR products were run on a 1\% agarose gel and analysed via the Amersham Imager 600 for the presence of one specific band approximating to the size of the target gene. 
qPCR

Quantitative PCR (qPCR) was performed on mammalian cell cDNA samples using Sso Advanced Universal SYBR Green Supermix (Bio-Rad) according to the manufacturer's instructions. Briefly, $5 \mu \mathrm{l}$ of the SsoAdvanced Universal SYBR Green supermix (2X) was combined with forward and reverse primers, $1 \mu \mathrm{g}$ of cDNA template and water to bring to the final reaction volume to $10 \mu \mathrm{L}$. The samples were run in the Bio-Rad CFX96 Real-Time PCR system. Using the calculated threshold cycle $\left(\mathrm{C}_{\mathrm{T}}\right)$, the Livak method was used to analyse relative expression of genes encoding IL-6 and IL-8. Using the housekeeping gene, Cyclophilin A $(\operatorname{cyclA})$, as a reference, and the untransfected, non-TNF- $\alpha$ stimulated HeLa 229 cell sample to normalise, the normalised expression ratio $\left(2^{-\Delta \Delta \mathrm{CT}}\right)$ was calculated. Melt curve analyses were conducted at the end of the run for each target gene, using the default conditions set by the BioRad CFX96 Real-Time PCR system. Data were analysed using Excel and Prism GraphPad.

\subsection{Human Cytokine Analysis}

The Proteome Profiler Human Cytokine Array kit (R\&D Systems, In Vitro Technologies) is a membrane-based sandwich immunoassay, with a nitrocellulose membrane spotted with an array of antibodies to 36 human cytokines and chemokines. Cell culture medium $(1 \mathrm{ml})$ and lysates $(700 \mu \mathrm{l})$ from transfected, TNF- $\alpha$-stimulated and unstimulated HeLa cell samples were prepared as described above. Before starting, reagents and buffers were prepared as outlined in the assay manual.

The nitrocellulose membranes were soaked in blocking buffer (Array Buffer 4) for 1 hour on a rocking platform shaker. Samples (culture medium or cell lysates) were thawed and prepared by combining with Array buffer 4 and 5, and Human Cytokine Array Detection Antibody Cocktail and incubating at room temperature for one hour. Array buffer 4 was removed from membranes and samples and antibody mixtures were applied to the prepared membranes and incubated overnight at $4^{\circ} \mathrm{C}$ on a rocking platform.

The following day, membranes were washed three times with the included wash buffer. Streptavidin-HRP was diluted in Array Buffer 5 and applied to the membranes. Membranes were incubated for 30 minutes with gentle shaking before being washed as before. The membranes were treated with a Chemi Reagent Mix, covered with a plastic sheet protector, and imaged with an Amersham Imager 600 (GE Life Sciences) with multiple exposure times used to obtain the best membrane exposure images. Profiles of mean spot pixel density were calculated using a transmission mode scanner and image analysis software installed on the 
Amersham Imager. Increasing the exposure time did not impact the pixel intensity of each cytokine or chemokine but did amplify the background intensity. Therefore, background was removed by subtracting the negative control value from each of the other spots. Data was further analysed using Microsoft Excel and Prism GraphPad. T tests were conducted to assess significance by comparing pixel intensity values of selected pairs.

\subsection{Bacterial Conjugations}

\section{Conjugations with E. coli recipient}

Test conjugations, using an E. coli strain as the recipient, were done to ensure that our E. coli $\mathrm{S} 17-1$ conjugation strain was competent to mobilise plasmids, and that our plasmids of interest contained intact origins of transfer. Our mobilisable plasmids, pSRKGm or derivatives of pEX18Gm, conferred resistance to gentamicin. To select transconjugants, our recipient $E$. coli strain harboured pACYC184, a plasmid with the p15a origin of replication, which is compatible with both pSRKGm and pEX18Gm plasmids. Because pACYC184 confers chloramphenicol resistance, transconjugants were selected on LB agar with both gentamicin and chloramphenicol. Conjugations were done essentially as described [119]. The recipient (pACYC184 strain) and donor (S17-1 harbouring pSRKGm or pEX18Gm derivative) strains were cultured separately overnight, with shaking, in LB broth with appropriate antibiotics. The next morning, $1 \mathrm{ml}$ of each overnight culture was centrifuged for one minute at top speed. The supernatant was removed, and each pellet was resuspended in $110 \mu 1$ of LB broth without any antibiotics added. For the test plate, $50 \mu \mathrm{l}$ of each donor and recipient were combined on a sterile nitrocellulose filter on a LB plate without any antibiotics. To be sure our antibiotics were working, for each conjugation, donor-only and recipient-only controls were each set up, with $50 \mu 1$ resuspended pellet on their own nitrocellulose filters and plates. The bacterial matings or controls were placed lid-side up in the $36^{\circ} \mathrm{C}$ incubator for 5-6 hours. After this, the nitrocellulose filters were transferred to $15-\mathrm{ml}$ conical tubes containing $2 \mathrm{ml}$ of LB without antibiotics. The bacteria were vortexed for one minute to dissociate all of the bacteria from the filters. For each bacterial suspension, $100 \mu \mathrm{l}$ was plated onto LB agar with gentamicin and chloramphenicol. Plates were incubated overnight at $36^{\circ} \mathrm{C}$.

\section{Conjugations with $B$. quintana recipient}

For generation of mutants, the BepA1 mutagenic plasmid was cultured in S17-1 in order to be mobilised into B. quintana. E. coli S17-1 carrying pSRKGm, a broad-host-range plasmid 
that is predicted to be able to replicate in B. quintana, was included as a positive control for conjugation, as we hypothesised that colonies would be obtained without the chromosomal integration required for selection of a suicide plasmid.

Prior to the conjugation, Bartonella quintana wild type JK-31 was cultured on nonselective chocolate agar plates. Plates with confluent bacterial growth, about 4-5 days after streaking, were used. Single colonies of S17-1 strains harbouring pEX18Gm_BepA1 or pSRKGm were cultured overnight with shaking in $5 \mathrm{~mL}$ LB broth with gentamicin. The following day, the S17-1 strains were removed from the incubator, diluted 1/20 in fresh LB with gentamicin, and returned to the shaking incubator for 1.5 to 2 hours, or until an $\mathrm{OD}_{600}$ of 0.25-0.5 was reached. In most cases, the cultures reached an $\mathrm{OD}_{600}$ of 0.4-0.5. For each strain, the amount of each S17-1 culture to use in the conjugation was calculated based on the final $\mathrm{OD}_{600}$ reading, according to the following formula:

$$
\mathrm{X}(\mu \mathrm{l})=125 / \mathrm{OD}_{600}
$$

The calculated amounts of S17-1 strains were transferred to new microcentrifuge tubes and the bacteria were pelleted by centrifugation at maximum speed for one minute. The pellet was washed three times with $1 \mathrm{~mL}$ M199S to remove all traces of antibiotics. After the last wash, the pellet was not resuspended. In the meantime, B. quintana JK-31 was harvested by scraping from plates and transferring into a fresh tube. Depending on bacterial growth, bacteria from 1-3 plates were combined. JK-31 was suspended in $1 \mathrm{ml}$ of M199S in a fresh microfuge tube, using gentle pipetting to resuspend the pellet. A 1/100 dilution was made into a fresh tube and the $\mathrm{OD}_{600}$ was measured and recorded. JK-31 was washed once, spinning at top speed for 2 minutes, then the pellet resuspended in a small volume (e.g., $250 \mu 1$ ) of M199S. For each conjugation, $100 \mu \mathrm{L}$ of the concentrated and washed JK-31 suspension was used to resuspend one of the S17-1 strain pellets, then both were pipetted onto the center of a non-selective chocolate agar plate. The plate was allowed to air dry in the hood for 5-10 minutes. Afterwards, plates were sealed lid-side up in a fresh and clean candle jar without dispersing the bacterial suspension. The candle was lit, the jar sealed, and plates were incubated for $6-8$ hours at $36^{\circ} \mathrm{C}$. The entire bacterial growth on the conjugation plates was scraped using a sterile loop and resuspended in $300 \mu \mathrm{L}$ M199S. Gentle pipetting removed the clumps and 1:5 dilutions were made by pipetting $100 \mu \mathrm{L}$ of each suspension to tubes containing $400 \mu \mathrm{L}$ of fresh M199S. From both the undiluted and diluted tubes, $100 \mu \mathrm{L}$ of bacterial suspension was plated onto chocolate plates supplemented with gentamicin (to select for plasmid maintenance or integration into the chromosome). The selective plates also included nalidixic acid $(20 \mu \mathrm{g} / \mathrm{ml})$ and cefazolin $(2 \mu \mathrm{g} / \mathrm{ml})$ as a counterselection against S17-1. The plates were placed lid down 
in the candle jar, the candle lit and placed in a $36^{\circ} \mathrm{C}$ incubator. Plates were checked after 1416 days for the appearance of colonies. 


\section{Chapter 3 BepA1 and Cytokine and Chemokine Expression in HeLa cells}

\subsection{Introduction}

Bacterial infection in the human host generally results in a rapid immune response, including the generation of cytokines and chemokines, small extracellular signalling molecules that are involved in inflammation, cell migration, cell growth and differentiation. Cytokines and chemokines are central modulators of homeostasis and the innate immune response [120]. Many pathogens, including viruses, bacteria, and protists, have evolved mechanisms to interfere with cytokine and chemokine signalling, enabling a prolonged or persistent infection. This is likely also true for a stealth pathogen such as B. quintana, which needs to persist in the bloodstream long enough for transmission to a new host via a body louse blood meal.

Cytokine and chemokine expression occurs following immune detection of microbial signatures, including PAMPs, and are controlled by central immune signalling pathways, including NF-KB. By looking at changes in the expression of multiple cytokines we can get an idea of the signalling pathways that could be disrupted by bacterial infection.

B. quintana lives in the bloodstream, invading the reservoir host's erythrocytes to enable transmission via blood-feeding arthropods. During infection of the bloodstream, the bacteria likely subvert inflammatory pathways to prolong the infection, and the suite of secreted intracellular effectors may play a part. This led us to hypothesise that a target of $B$. quintana BepA1 could be a signalling protein important in innate immunity and infection.

A former student in the lab transfected HeLa cells with the wild type BepA1 protein or an empty pcDNA3.1 vector control, stimulated with TNF- $\alpha$, and then conducted ELISA experiments to see if BepA1 suppressed the expression of IL-6 or IL-8. His data suggested that IL-6 and IL-8 expression were both suppressed by BepA1. However, these experiments also showed that transfection with an empty plasmid, followed by TNF- $\alpha$ stimulation induced higher cytokine production than TNF- $\alpha$ stimulation alone, suggesting that the introduction of dsDNA into the cytoplasm or the transfection protocol can trigger innate immune reactions $[113,121]$.

To expand on and clarify these preliminary findings, we asked whether the FIC domain was necessary for BepA1-mediated cytokine suppression. First, a control plasmid, identical to the wild type BepA1 version apart from a point mutation in a residue essential for AMPylation, was generated. This was included to determine whether AMPylation activity in BepA1 was required for the suppression of the cytokines. Second, we selected a screen to see if the expression of additional cytokines and chemokines were altered by BepA1. Because the innate 
immune response involves the coordinated effects of multiple cytokines and chemokines, we wanted to determine how wide-ranging this inhibition might be. This data would provide insights into the signalling pathways affected by BepA1-mediated AMPylation.

To test the expression of a panel of cytokines, we utilised a Proteome Profiler Human Cytokine Array Assay (R\&D Systems). This assay enables four samples to be simultaneously tested for the expression of 36 different cytokines and chemokines, which are captured by duplicate antibodies spotted on a membrane. In this experiment, we transfected HeLa cells with BepA1 wild type and a catalytically dead mutant version, in which the conserved FIC domain histidine is converted to an alanine, abolishing AMPylation activity. Both of these transfected HeLa cells were also stimulated with TNF- $\alpha$.

Additionally, we included two untransfected HeLa cell samples, one to serve as our basal control, representing the intrinsic resting immune state of HeLa cells, and one which was stimulated with TNF- $\alpha$. TNF- $\alpha$ is also a potent proinflammatory cytokine that induces the expression of proinflammatory genes via the TNF- $\alpha$ receptor, which activates both the MAPK and NF-KB signalling pathways.

Of these HeLa cell samples, we tested both the cell medium and the cell lysate with the Proteome Profiler assay. This was to detect both secreted cytokines and chemokines, as well as those that are retained in the cell cytoplasm or on the cell surface.

The aim of this experiment was to compare cytokine responses of human HeLa-229 cells following TNF- $\alpha$ stimulation, and to determine whether BepA1 could alter these. Inclusion of the FIC domain mutant allowed us to better understand whether AMPylation of host cell proteins impacts NF- $\mathrm{BB}$ or MAP kinase signalling pathways. 


\subsection{Results}

Infection models are a useful tool to study bacterial pathogenesis, including those replicate the course of infection in a relevant host or cell line. Animal infection models are challenging with $B$. quintana, as they are limited to human volunteer studies or primates such as rhesus macaques. Tissue culture models have been used, but B. quintana is challenging to genetically manipulate, making it difficult to study isogenic mutants. As an alternative to cell infection with a BepA1 mutant, we opted to assess the impact of BepA1 on innate immunity by transfecting the gene into HeLa-229 cells.

HeLa cells transfected with the wild type version of BepA1 or a catalytically inert point mutation (i.e., the FIC domain mutant) were treated with the proinflammatory cytokine, TNF$\alpha$, to elicit a potent immune response. HeLa-229 cells release low levels of proinflammatory cytokines in the resting state which made HeLa cells the ideal cell-line for these experiments. Transfection with the WT BepA1 mutant or the FIC domain mutant would not result in such an exaggerated immune response, as observed in other cell lines e.g. HEK293, and only allow us to observe the effects of the individual proteins, therefore, making the results would be more reliable.

TNF- $\alpha$ binds to its main receptor, TNFR1, which initiates a signalling cascade that results in cell survival via the activation of the transcription factor NF-KB. However, TNF- $\alpha$ can also result in the activation of apoptosis and necrosis and when checkpoints are disrupted, cell death occurs [122]. We hypothesised that WT BepA1 may be AMPylating and altering the function of a signalling protein in this pathway that results in altered cytokine and chemokine expression while also preventing programmed cell death (apoptosis) by some yet unknown mechanism.

We included two non-transfected HeLa cell controls for this study, comparing the cytokine and chemokine expression profile of unstimulated versus TNF- $\alpha$ stimulated cells. These controls served to demonstrate which cytokines and chemokines were responsive to TNF- $\alpha$ stimulation in the absence of plasmid transfection. The inclusion of the FIC domain mutant BepA1 protein was also crucial as it allowed us to confirm whether AMPylation resulted in altered host cell signalling.

We opted to test both cell medium and cell lysates because some proteins on the array, such as Intercellular Adhesion Molecule 1, ICAM-1, are frequently found on the cell surface. Other cytokines, such as IL-1 family members, are synthesised as precursors that are intracellular. 
Each Proteome Profiler membrane was exposed multiple times for different durations, but the best images (highest signal to background) were seen with exposures of 4 minutes, shown in Figure 3.1.

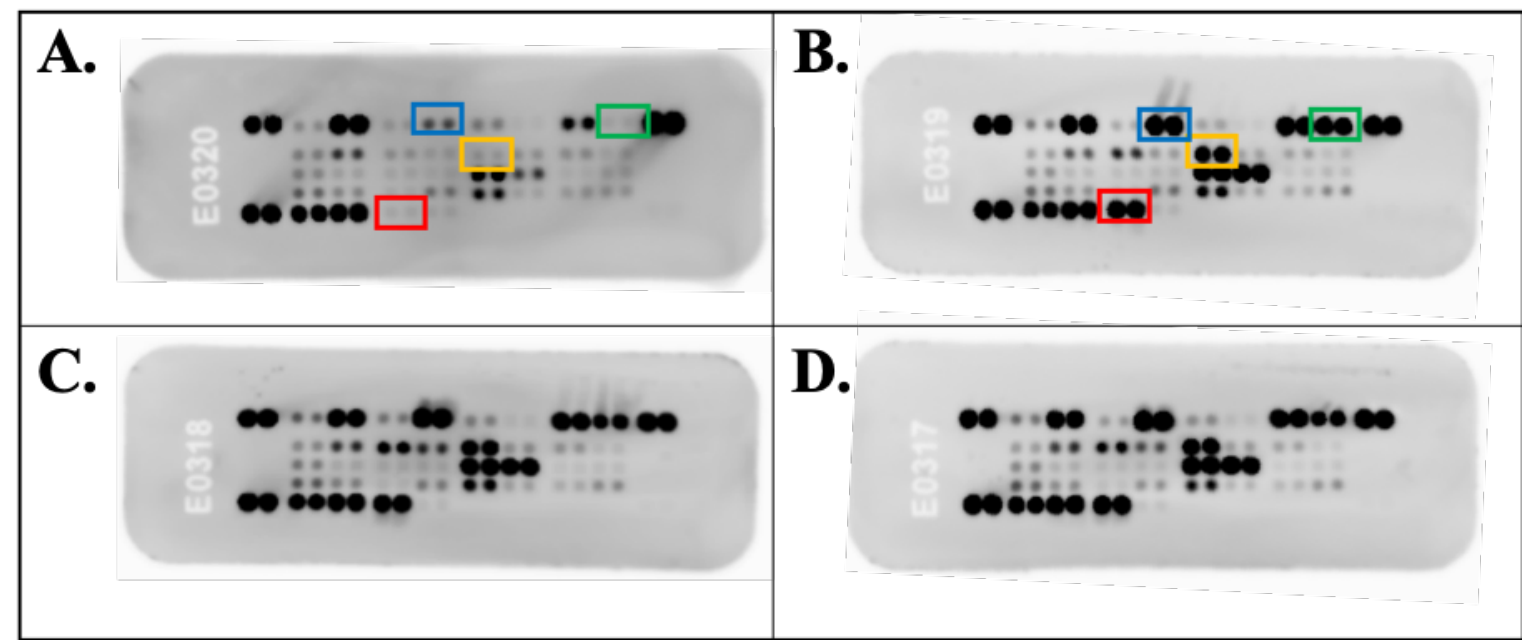

Figure 3.1 Proteome Profiler Human Cytokine Array exposed membranes for cell medium using one biological replicate. The exposed membrane images are shown for all four HeLa cells sample. (A) HeLa basal (untransfected, no TNF- $\alpha$ treatment); (B) HeLa cell untransfected, TNF- $\alpha$ treated. (C) BepA1 WT transfected, TNF- $\alpha$ treated, and (D) FIC domain mutant transfected, TNF- $\alpha$ treated. The membranes were exposed for 4 minutes each. Spots representing cytokines that are induced by TNF- $\alpha$ are indicated by coloured boxes and include CCL5/RANTES (blue), ICAM-1 (yellow), and CXCL10/IP-1 (green). TNF- $\alpha$, both endogenous and added, is indicated by the red box.

The membrane images showed several spots with varying degrees of intensity. Each represents a cytokine or chemokine, spotted in duplicate. The darker spots represent cytokines and chemokines which have been expressed at a higher level than the spots with lighter intensities. Figure 3.1 reveals a clear difference in expression of multiple cytokines and chemokines. The HeLa basal membrane (Fig. 3.1 A) shows several cytokines that are secreted at high levels, likely these help maintain basic function of the cell. Some of the cytokines with high basal expression include CCL2/MCP-1, IL-6, MIF, and serpin E1/PAI-1 (several of the spots are for reference). Treatment with TNF- $\alpha$ results in higher levels for several cytokines, as indicated by the coloured outlines. Differences between HeLa cells transfected with BepA1 WT, or the FIC domain mutant, are more subtle, but are still present.

The annotated membranes with the cytokines and chemokine coordinates are shown in Figure 7.3, while all of the scanned membranes are shown in Figure 7.4 (both in Appendix). 


\section{Cytokine and chemokine secretion in cell mediums and cell lysates}

To quantify the differences observed, the pixel intensity of each spot was determined, and the background subtracted. Duplicates were averaged and plotted. The data is summarised for the secreted cytokines (cell medium) in Figure 3.2, and for the cell lysates in Figure 3.3. The pixel intensity values are provided in Appendix Tables 7.5.1 and 7.5.2.

Comparing cytokine expression in the untransfected HeLa cells, with or without TNF$\alpha$ added (see green and blue bars in Figs 3.2 and 3.3), there are several cytokines and chemokines that are induced by TNF- $\alpha$, as expected. HeLa cells generally express low levels of pro-inflammatory cytokines in the resting state, but cytokines such as CXCL1/GRO $\alpha$, IL-6 and IL-8 all increase following TNF- $\alpha$ treatment, in both cell medium and the cell lysates. In the cell medium, the secreted cytokines CCL5/RANTES, CXCL10/IP-10, and ICAM-1/CD-54 all increase with the TNF- $\alpha$ treatment, while an increase in IL- $1 \alpha / \mathrm{IL}-1 \mathrm{~F} 1$ is seen in the cell lysates only. TNF- $\alpha$ itself is increased in the cell medium, although this assay does not distinguish between endogenous TNF- $\alpha$ and that added to the cells. All the cytokines and chemokines which have increased expression after TNF- $\alpha$ treatment, are molecules which are likely under the control of NF- $\kappa B$ or other transcription factors activated by TNF- $\alpha$, such as p38, JNK, or AP-1. These data are in line with what would be predicted following TNF- $\alpha-$ mediated immune activation in HeLa-229 epithelial cells.

HeLa cells transfected with either wild type BepA1 or the FIC domain mutant and stimulated with TNF- $\alpha$ were also evaluated, with the average pixel intensities from cell media shown in Figure 3.2 and cell lysates in Figure 3.3 (comparing pink and purple bars). The expression of multiple cytokines and chemokines differed between cells transfected with the BepA1 WT protein vs the FIC domain mutant. As our preliminary data suggested that BepA1 could be suppressing the secretion of cytokines such as IL-6 and IL-8, we were particularly interested in cytokines that were secreted at higher levels in cells transfected with the FIC mutant, compared to those transfected with wild type BepA1, as those two types of cells should only differ in the presence of an active bacterial AMPylation enzyme. Expression levels of cytokines that showed this pattern for the BepA1 WT and FIC mutant-transfected cells are shown in Figure 3.4, including both cell medium (A) and lysates (B).

Three cytokines, IL-6, IL-8, and Serpin E1/PAI, are all present at higher levels in both cell medium and lysates for cells transfected with the FIC mutant, relative to the BepA1 WT (Figure 3.4). In addition, CCL2/MCP-1, CCL5/RANTES, CXCL1/GRO $\alpha$, CXCL10/IP-10, MIF, and TNF- $\alpha$ are all present at higher levels in the cell medium of cells transfected with the 
FIC mutant, relative to BepA1 WT. For the cell lysates, higher levels of IL-32 $\alpha$ are seen with cells transfected with the FIC mutant.

\section{Pixel intensity values for cytokines and chemokines in HeLa cell medium}

A.

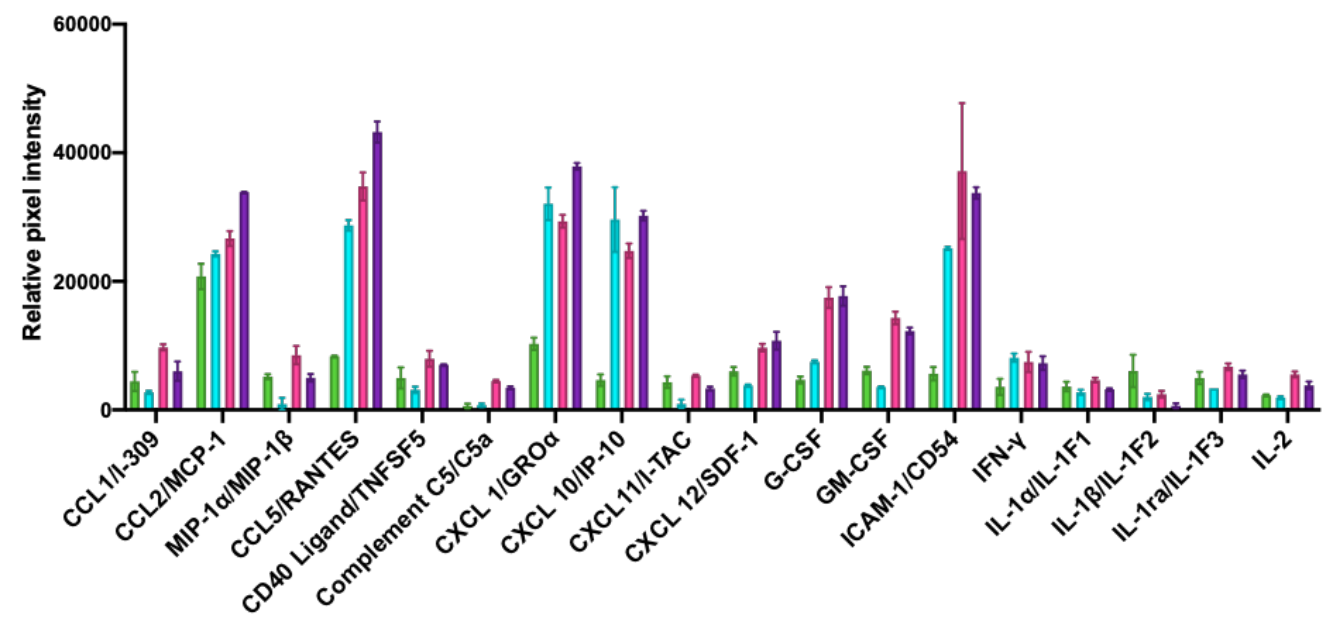

B.

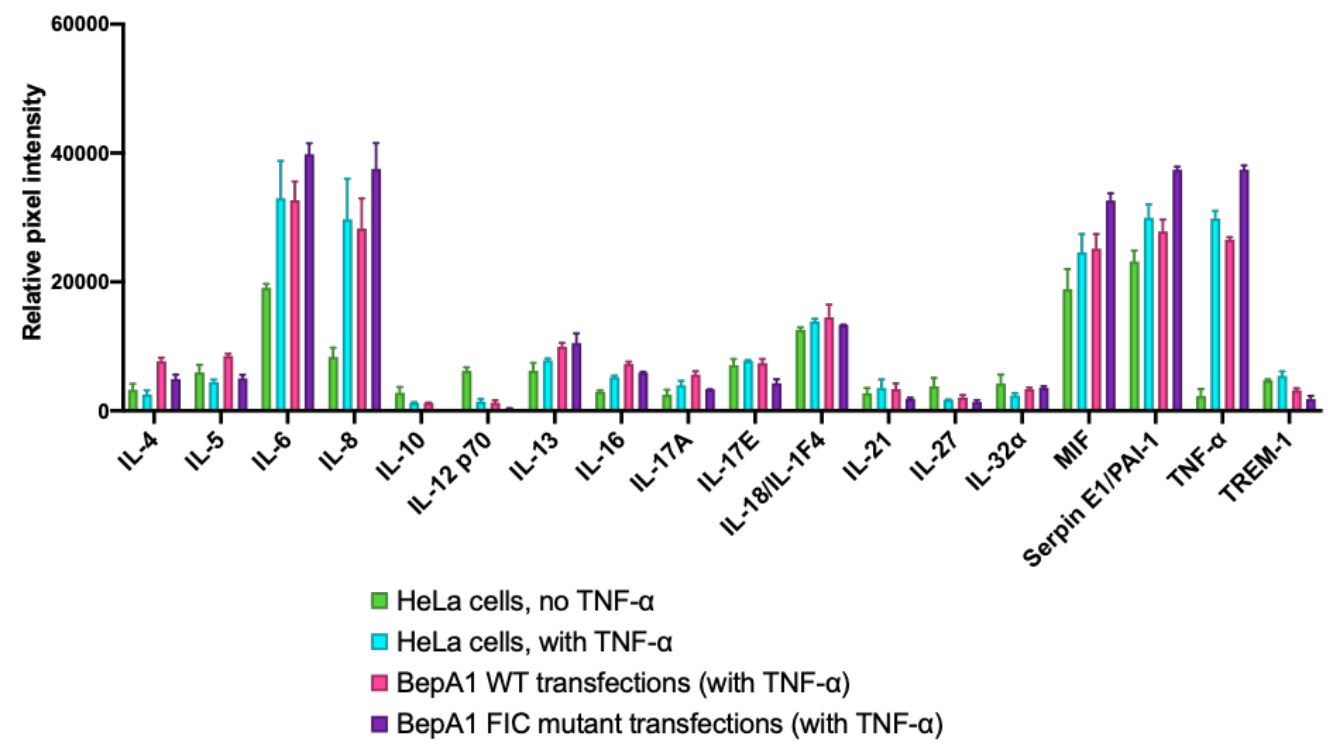

Figure 3.2 Average pixel intensity values for all 36 cytokines and chemokines tested in all four cell samples for cell medium, using one biological replicate per sample. Cell medium tested with the Proteome Profiler Human Cytokine Array showed treatment with TNF- $\alpha$ altered the secretion of many cytokines and chemokines in HeLa cells that were untransfected (green and blue bars) or transfected with the BepA1 wild type (pink bars) or with the BepA1 FIC domain point mutant (purple bars). Error bars show the average standard deviation for the two samples of each cytokine and chemokine. 
A.

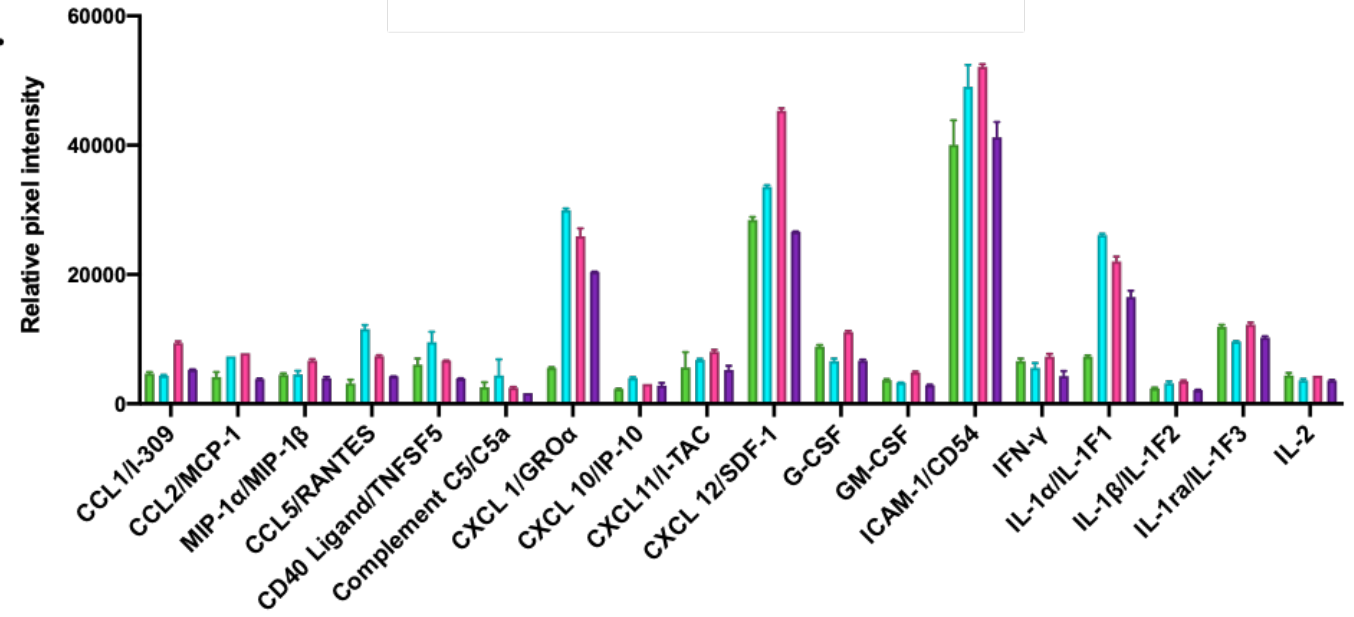

B.

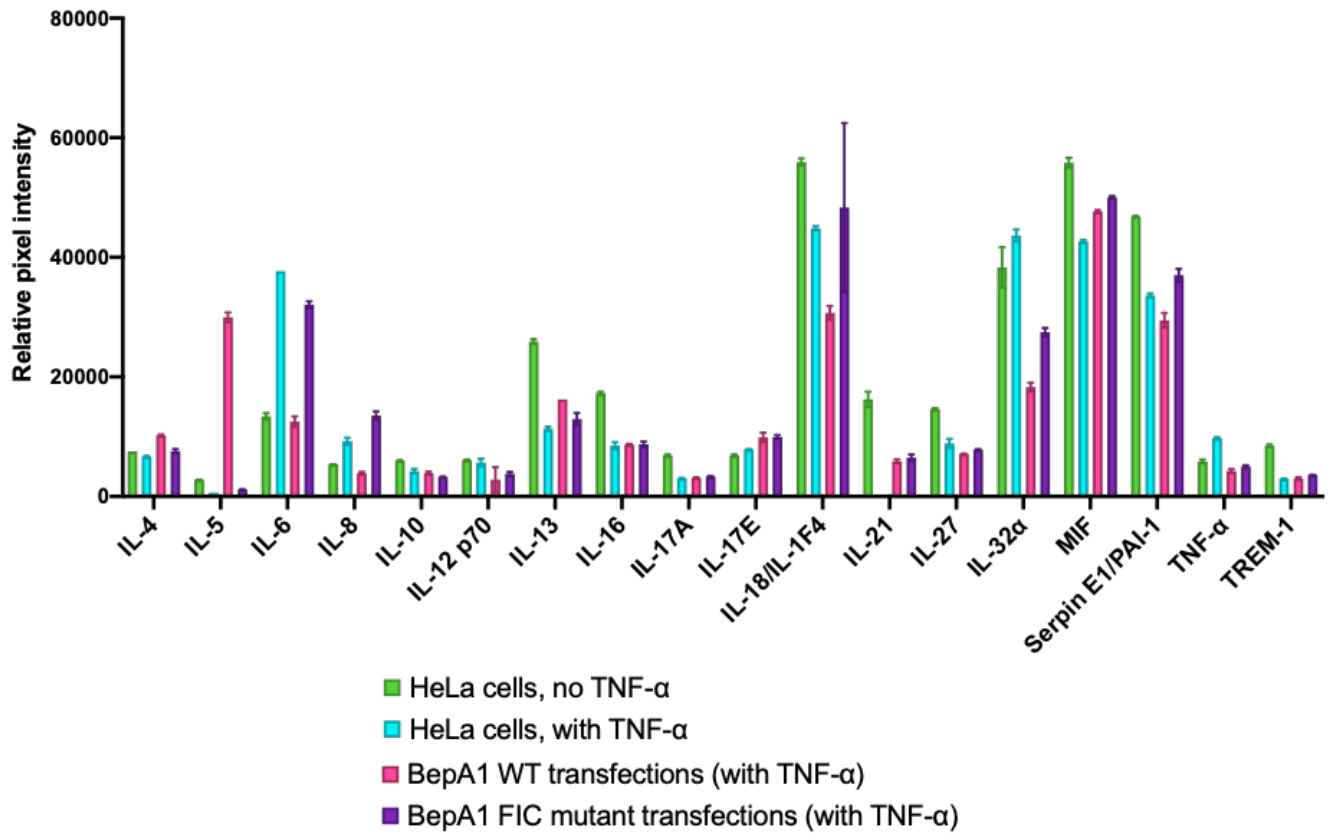

Figure 3.3 Average pixel intensity values for all 36 cytokines and chemokines tested in all four cell samples for cell lysates, using one biological replicate per sample. Cell lysates were prepared and tested using a Proteome Profiler Human Cytokine Array to determine the expression of multiple cytokines and chemokines. The treatment with TNF- $\alpha$ altered the expression of many cytokines and chemokines in untransfected HeLa cells (green bars, no TNF- $\alpha$, blue bars, after one-hour TNF- $\alpha$. HeLa cells were also transfected with wild type BepA1 (pink bars) or a catalytically inert FIC domain mutant (purple bars). Error bars show the average standard deviation for the two samples of each cytokine and chemokine. 
A.

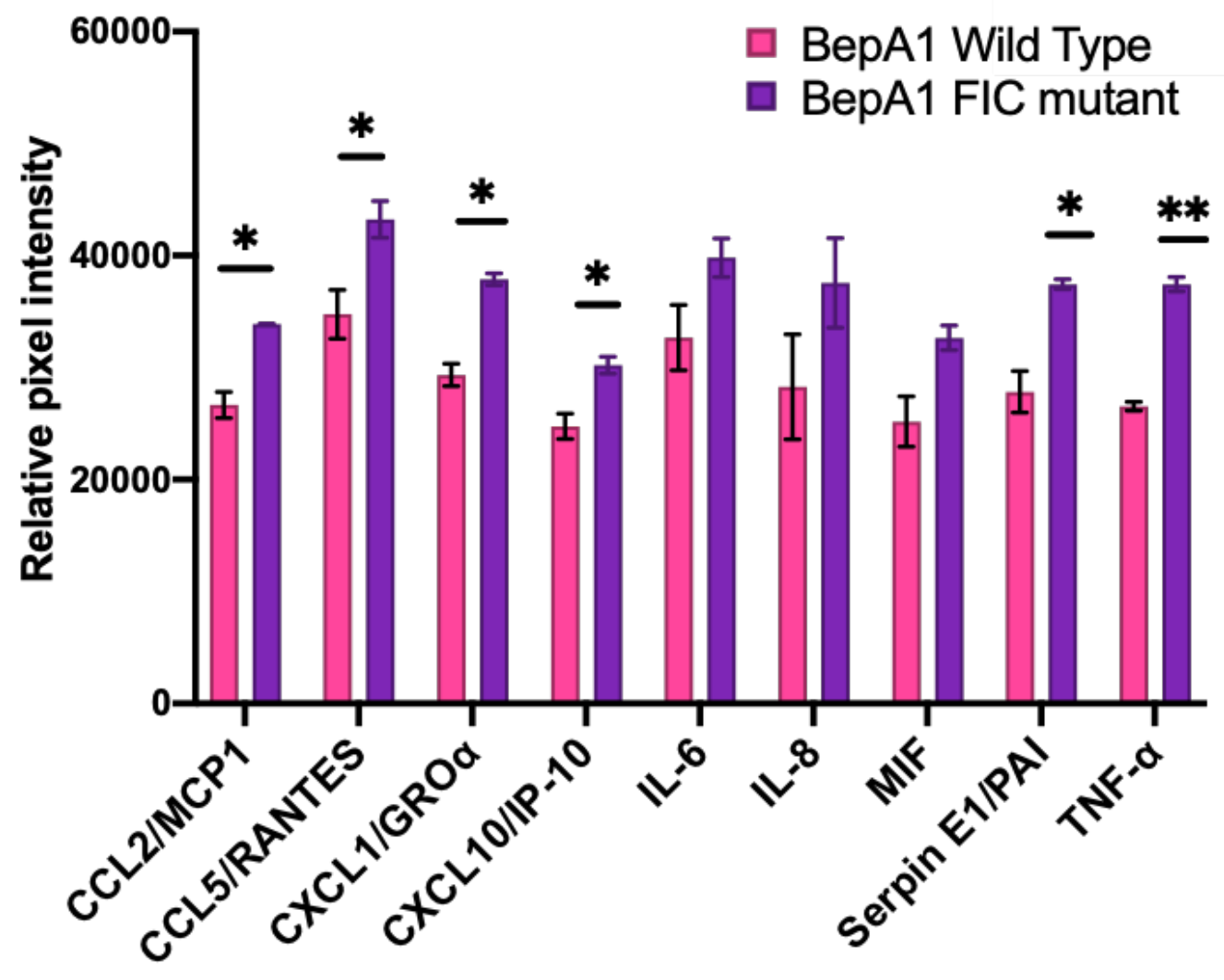

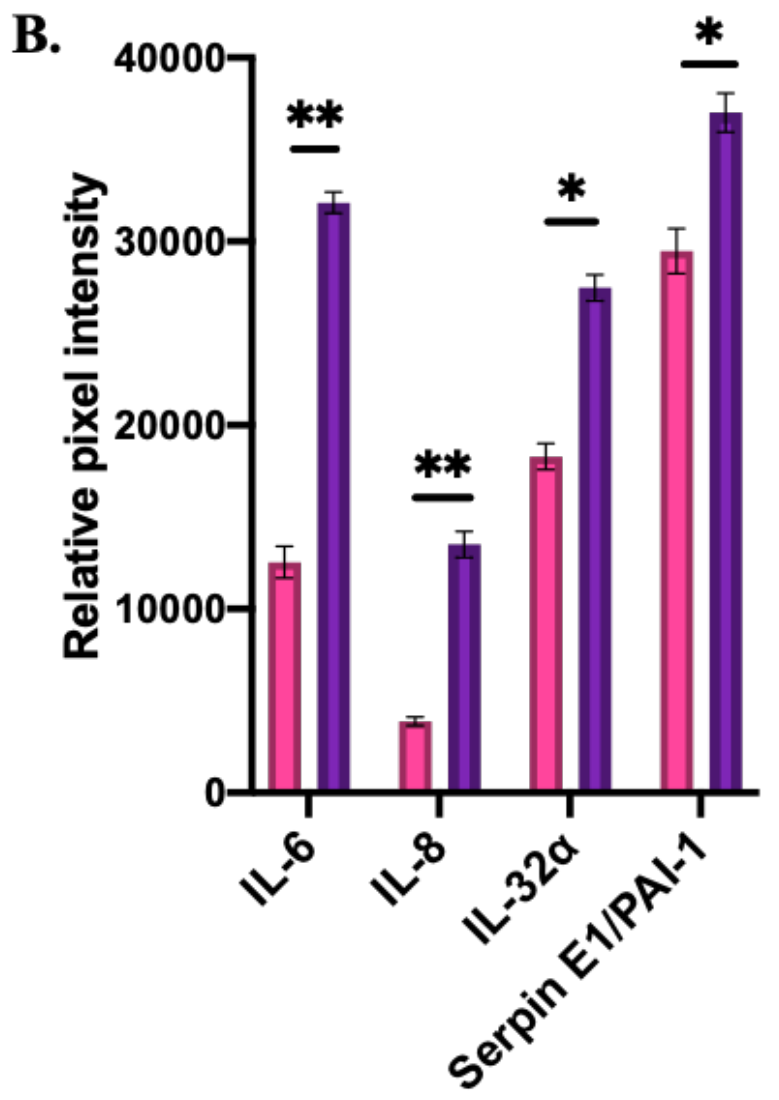

Figure 3.4 Mean pixel intensities of selected cytokines and chemokines in BepA1 WT and FIC domain mutant TNF- $\alpha$ treated cell medium samples, and cell lysates. Selected cytokines and chemokines from HeLa cells transfected with BepA1 WT (pink bars) or the FIC domain mutant (purple bars) are shown for cell medium (A) or cell lysates (B). Mean pixel intensities were determined for duplicate spots, Pairwise T tests were done with GraphPad Prism, comparing values from BepA1 WT vs the FIC domain mutant. * $*$ p value $<0.05$ and $* *=p$ value $<0.005$ comparing BepA1 WT protein and FIC domain mutant protein transfected, TNF- $\alpha$ stimulated HeLa cells. Error bars show the average standard deviation for the two samples of each cytokine and chemokine. 


\subsection{Discussion}

Bacteria entering the human host are recognised by the innate immune system through their PAMPs, which result in the activation of pattern recognition receptor, including Toll-like receptors (TLRs) and nucleotide-binding oligomerization domain-like receptors (NLRs). These signals initiate an intracellular signalling cascade that amplifies the immune response against pathogens. This response includes activation of gene expression, secretion of a wide range of cytokines, chemokines, cell adherence proteins and recruitment of immune cells to the site of infection, working in combination to coordinate the initial host immune response [123].

Many bacterial and viral pathogens can combat the innate immune response via many different mechanisms, but many manipulate cytokine and chemokine networks, particularly by inhibiting their synthesis or secretion. This can be achieved by via the inhibition of signalling pathways, e.g., MAPK pathway or NF-кB-mediated proinflammatory responses, which are activated downstream of TLR and NLR activation [124]. Bartonella spp. are unique pathogens, utilising a complicated infection strategy that results in prolonged and persistent infection in the host bloodstream. Bartonella likely avoid some immune detection by the host through mechanisms not yet characterised. Much remains unknown about their interactions with innate immune cells, including macrophages and neutrophils, or localised interactions with vascular endothelial cells.

B. quintana is known to manipulate host cytokine expression in patients with chronic bacteraemia. Capo et al., showed that macrophages collected from homeless people who were bacteraemia with $B$. quintana, produced specific increases in secretion of the anti-inflammatory cytokine IL-10, relative to homeless people without $B$. quintana infection [125]. IL-10 primarily targets leukocytes and is a master negative regulator of inflammation, often produced toward the end of an infection [126, 127]. Inducing IL-10 expression leads to the general dampening of the immune response, which may facilitate B. quintana infection. However, the mechanism by which $B$. quintana manipulates IL-10 expression remains unknown.

Our experiments offer the first experimental evidence that suggests B. quintana may manipulate proinflammatory cytokine and chemokine expression via a secreted bacterial effector. Avoidance of immune recognition is essential for persisting within the host and transmission to new hosts. However, there is a wide variety of mechanisms by which host immunity can be suppressed, evaded or manipulated. Our work is the first to suggest that a bacterial effector with a FIC domain is involved in manipulation of cytokine and chemokine expression. 
Our transfection model enabled us to characterise cytokine and chemokine production and secretion by HeLa-229 cells transfected with the B. quintana WT BepA1 protein or a catalytically dead BepA1 FIC domain protein. These experiments were designed to compare samples that differed only by one variable - the presence of a catalytically active FIC domain, which is why we focused on the comparison between these two samples. Previous studies had suggested that the B. quintana WT BepA1 protein significantly suppressed the secretion of IL6 and IL-8 in transfected HeLa cells relative to cells transfected with an empty vector [113].

The majority of the cytokines that were expressed at a lower level in the presence of the WT BepA1 protein are chemotactic cytokines (i.e., chemokines) that attract a wide range of innate immune effector cells to the site of infection. These included CCL2/MCP-1 and CXCL10/IP-10, both of which attract macrophages and monocytes, T lymphocytes and natural killer (NK) cells [128-131]. Both CCL5/RANTES and MIF are involved in leukocyte recruitment, and MIF also primes endothelial cells for an immune response by amplifying cytokine release and upregulating the expression of surface adhesion molecules to enable the immune cells to "stick" [132, 133]. Both CXCL1/GRO $\alpha$ and IL-8 are chemotactic for neutrophils, the most abundant immune cells in the bloodstream [134-136] . They further activate neutrophils by promoting degranulation, the release of an armament of antimicrobial factors. The presence of a WT BepA1 also resulted in reduced expression of more pleiotropic pro-inflammatory factors, including IL-6, which is a general marker of inflammation [137]. After synthesis at the site of infection, IL-6 travels through the bloodstream and stimulates the synthesis of multiple inflammatory markers, including acute phase proteins, in the liver [137]. TNF- $\alpha$ is another pleiotropic pro-inflammatory cytokine, ramping up the immune response and triggering the production of additional pro-inflammatory cytokines [138, 139]. Together, these findings are consistent with our hypothesis that B. quintana embeds itself in the vascular system, and that endothelial cells are the target of the secreted effectors. By dampening down the synthesis of chemokines, the bacteria may alter or delay the recruitment of immune cells to the site of infection and promote their own persistence.

These cytokines and chemokines target a wide range of cells, immune and non-immune, and signal through multiple pathways. By suppressing the secretion of these molecules, $B$. quintana may be affecting multiple cell types and protein functions. Down-regulating chemokines could be advantageous for the bacterium, preventing immune cell migration to the site of infection and delaying detection or removal by the immune system. 
The Proteome Profiler Human Cytokine array, combined with a transfection model, has limitations. Each cytokine was measured in duplicate for each sample. This corresponds to only two technical replicates and only one biological replicate. These results could be further validated by increasing the number of replicates and assessing cytokine expression via different

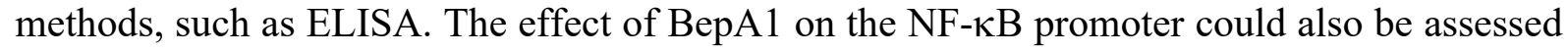

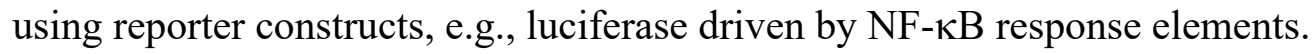

Another potential limitation is the method of transfection via Lipofectamine 3000. Lipofectamine is a cationic liposome formulation that forms a complex with nucleic acid, facilitating their contact with and uptake by the cell [140]. This process mimics some types of viral infection, and the foreign DNA is sensed by cytosolic sensors that are part of the innate immune response. We therefore found that cytokine secretion in cells transfected with BepA1 could not be directly compared to that of non-transfected cells.

An alternative approach, that is not confounded by triggering the innate immune system by transfection, would involve an in vitro infection model. In this case, HeLa-229 cells would be infected with either the WT B. quintana JK-31 and an isogenic BepA1 and BepA2 mutant. The roles of BepA1 and BepA2 could be elucidated by complementation with the genes, individually or together. Cytokine production, NF- $\mathrm{kB}$ activation, and the innate immune response, could then be elucidated. This experiment could also be carried out using endothelial cells, either primary cell lines, e.g., human microvascular endothelial cells, or immortalised cell lines, e.g., EA.hy926 cells. For this reason, I worked on generating the isogenic B. quintana BepA1 mutant.

Both BepA1 WT and the FIC domain mutant genes were cloned into pcDNA3.1 and transfected alongside a positive control plasmid, pcDNA3.1 that carried the B. quintana yopJ gene fused to a GFP tag. The control plasmid and the two BepA1 pcDNA3.1 plasmids are of similar sizes, suggesting transfection efficiency would not have significantly differed. With the positive control, $>50 \%$ of cells were observed fluorescing after transfection. Combined, these factors provided us with the confidence that BepA1 WT and the FIC domain mutant were successfully transfected into HeLa cells and resulted in expression.

Cytokine and chemokine networks are undoubtedly complex and are not completely understood. Our proteome profiler assay provides intriguing preliminary data that suggests that the B. quintana BepA1 WT protein reduces the expression of proinflammatory cytokines and chemokines, in comparison to a catalytically inert mutant variant. This work provides the first experimental evidence that a FIC domain containing Bartonella effector protein plays such a 
role. While there is still much to learn, including the identity of the host protein targeted by the B. quintana BepA1 protein, this research opens numerous doors to further study how this stealth pathogen is able to persist in vivo. 


\section{Chapter 4 Reverse Transcriptase and Quantitative PCR}

\subsection{Introduction}

Quantitative PCR, or qPCR, is a common tool used to detect and quantify the expression of target genes in a wide range of samples from many sources e.g. tissue, blood and cultured cells from plants, animals, humans and bacteria [141, 142]. qPCR is a fast and easy method of target gene quantification and detection of target genes is sensitive and specific, allowing researchers to perform high throughput target gene quantification $[142,143]$.

qPCR allows quantification of starting material - RNA, cDNA or DNA - through the use of fluorophores. These fluorophores can be either a fluorescent dye or fluorochromes and the choice of which fluorophore to use is dependent on available equipment and how much starting material there is. Our method of choice was a fluorescence dye-based quantification using the Sso Advanced Universal SYBR Green Supermix from Bio-Rad. While fluorescence dye-based quantification is not the most specific technique, it is simple and requires only a few reagents. Furthermore, it is rapid, with qPCR reactions typically being completed within 1 hour. A well-designed qPCR assay will be specific and sensitive for one target, where even few copies of starting RNA or cDNA can be used [144].

SYBR green is a fluorescent double-stranded DNA-binding dye that binds DNA on the minor groove, resulting in a structural change which releases energy that is detected by the qPCR machine as fluorescence. At the beginning of the qPCR reaction, the copy number of DNA amplicons are low, and it is difficult to distinguish between the fluorescence intensity of the reaction and background signal. However, as the reaction progresses, the number of DNA amplicons increases per cycle and the fluorescence intensity increases to detectable levels. In the end, the output is seen as an amplification plot, where fluorescence intensity is plotted against cycle number, representing the accumulation of DNA amplicons over the duration of the $\mathrm{qPCR}$ reaction.

As a technique, qPCR has many applications from gene expression profiling to assess the relative abundance of transcripts between samples, to viral titre determination to quantify viral copy number in samples. Our application was for gene expression profiling. Additionally, we used a two-step qPCR approach for our experiments where RNA was first reverse transcribed and followed by cDNA amplification and quantification. Separating these two steps has two main benefits. Firstly, we were able to use an exact amount of cDNA per reaction, and to save the rest, thus ensuring that we didn't run out of template. Secondly, two-step qPCR has a higher sensitivity, as we performed the reactions in individually optimised buffers. 
Our PCR-based assays had three goals: first, we wanted to validate the results we had seen with our Proteome Profiler Human Cytokine Array. This array tested for the presence of 36 cytokines, and we identified nine of interest that differed between cells transfected with WT BepA1 vs a catalytic inert FIC domain mutant. We opted to assess the expression of two of these signalling molecules, IL-6 and IL-8, as these are central innate immune modulators [113]. IL-8 is chemotactic for neutrophils and IL-6 modulates multiple innate inflammatory responses. Validating the expression of these cytokines would give additional information about the expression of these genes and provide quantifiable data to conduct a statistical analysis.

Secondly, we wanted to test for the gene expression of SM22, another protein not previously tested with the Proteome Profiler Assay. The gene encoding SM22, also known as Transgelin, is under the direct transcriptional regulation of Serum Response Factor (SRF). SRF is a transcription factor activated by Myozap and may lead to downstream activation of NF-KB [109]. Differences in SM22 gene expression in the BepA1 WT vs FIC domain mutant transfected HeLa cells would be expected if Myozap is targeted by WT BepA1.

Finally, we also carried out semi-quantitative reverse transcriptase PCR to answer the question of whether HeLa cells produce Myozap. A previous student's work identified a potential interaction between BepA1 and Myozap via a Yeast-2-Hybrid screen [113]. This interaction has not been validated, but before pursuing protein interaction studies, we first wanted to know if HeLa cells transcribe Myozap mRNA and produce the protein. This would set the stage for future interaction studies between Myozap and BepA1. 


\subsection{Results}

\subsubsection{Sample preparation and confirmation}

In order to compare expression levels of our target genes, HeLa cells were transfected with either WT BepA1 or the catalytically dead FIC domain mutant. Untransfected HeLa cells were also included in this study, as mammalian expression vectors may activate some pathways of innate immune signalling via cytosolic DNA sensors. After stimulation with TNF- $\alpha$ for onehour at $37^{\circ} \mathrm{C}$, total cellular RNA was purified and quantified as previously described (Methods, RNA Purification). The quantification data is summarised in Table 4.1 below. Additionally, we conducted a gel electrophoresis analysis of our purified RNA samples to check integrity and for the presence of the 28S and 18S RNA bands (not shown).

\begin{tabular}{|l|c|c|c|}
\hline \multicolumn{1}{|c|}{ RNA sample } & Concentration ng/ $\mathbf{\mu L}$ & $\mathbf{2 6 0 : 2 8 0}$ & $\mathbf{2 6 0 : 2 3 0}$ \\
\hline HeLa untransfected unstimulated (basal) & 278.0 & 2.00 & 2.00 \\
\hline HeLa untransfected TNF- $\alpha$ stimulated & 285.6 & 2.02 & 2.00 \\
\hline BepA1 WT unstimulated & 294.3 & 2.03 & 2.15 \\
\hline BepA1 WT TNF- $\alpha$ stimulated & 493.3 & 2.04 & 2.17 \\
\hline FIC domain mutant unstimulated & 320.0 & 2.00 & 2.08 \\
\hline FIC domain mutant TNF- $\alpha$ stimulated & 313.9 & 2.01 & 2.14 \\
\hline
\end{tabular}

Table 4.1 NanoDrop spectrophotometer concentrations, 260:280 and 260:230 ratios of RNA samples. The RNA concentrations, 260:280 and 260:230 ratios are summarized for all RNA samples.

We next synthesised cDNA, using the iScript Advanced cDNA Synthesis Kit for RTPCR as previously described (Methods, cDNA Synthesis). cDNA was quantified via a NanoDrop spectrophotometer and analysed via gel electrophoresis and can be seen in Figure 4.1 and Table 4.2 below.

\begin{tabular}{|l|c|c|c|}
\hline \multicolumn{1}{|c|}{ cDNA sample } & Concentration ng/ $\mathbf{\mu L}$ & $\mathbf{2 6 0 : 2 8 0}$ & $\mathbf{2 6 0 : 2 3 0}$ \\
\hline $\begin{array}{l}\text { HeLa untransfected unstimulated } \\
\text { (basal) }\end{array}$ & 1247.0 & 1.79 & 2.19 \\
\hline HeLa untransfected TNF- $\alpha$ stimulated & 1261.0 & 1.79 & 2.16 \\
\hline BepA1 WT unstimulated & 1230.3 & 1.80 & 2.29 \\
\hline BepA1 TNF- $\alpha$ stimulated & 1367.1 & 1.79 & 2.19 \\
\hline FIC domain mutant unstimulated & 1313.1 & 1.79 & 1.80 \\
\hline FIC domain mutant TNF- $\alpha$ stimulated & 1291.1 & 2.19 & 2.20 \\
\hline
\end{tabular}

Table 4.2 NanoDrop concentrations, 260:280 and 260:230 ratios are summarised for all cDNA samples. cDNA concentrations are fairly similar and do not show large within sample differences. 


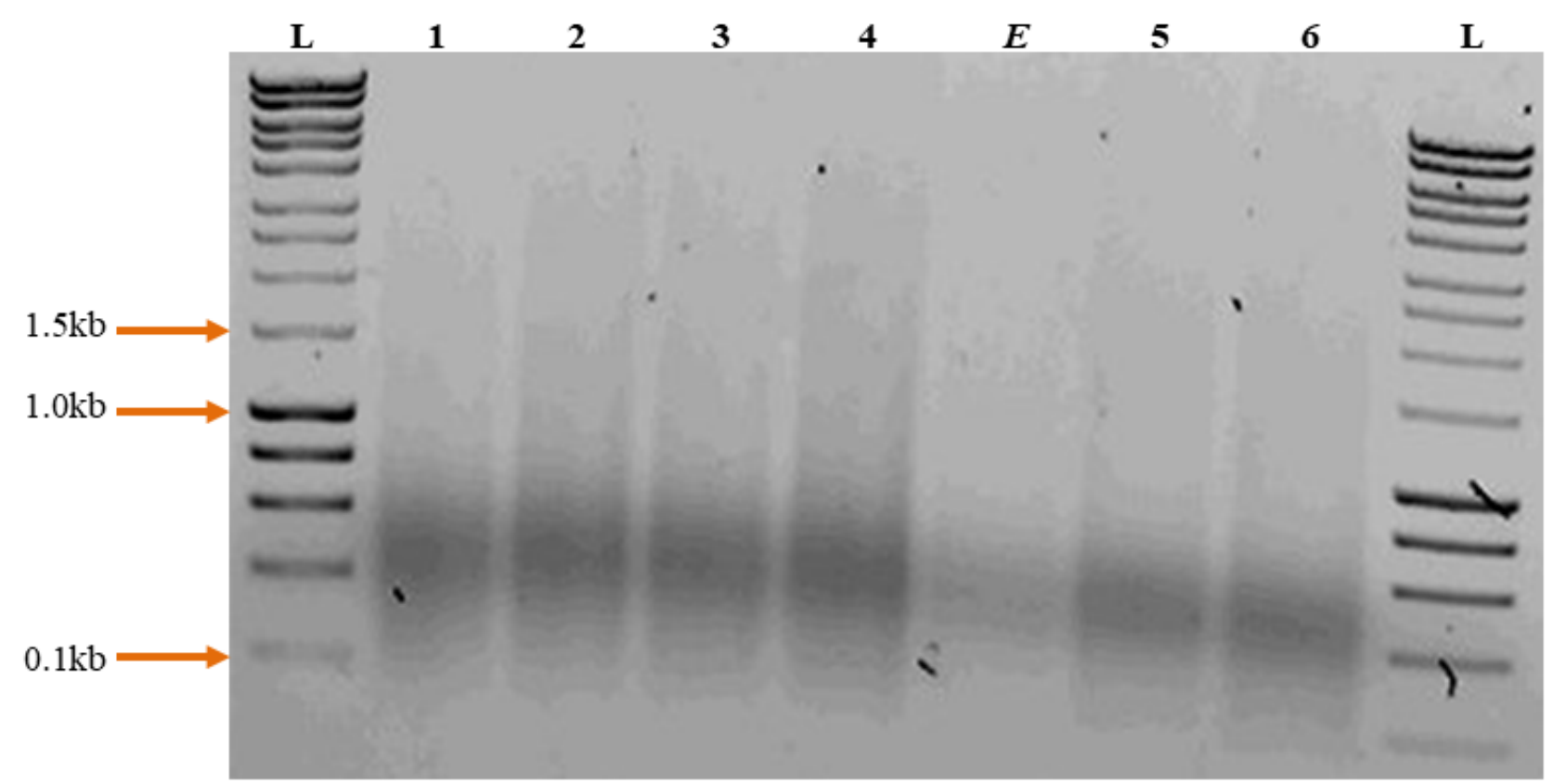

Figure 4.1 Gel analysis of cDNA samples. The cDNA is generally seen as a large smear. Lane 1: HeLa basal (untransfected, not TNF- $\alpha$ stimulated) cDNA. Lane 2: HeLa untransfected, TNF- $\alpha$ stimulated cDNA. Lane 3: BepA1 WT transfected, not TNF- $\alpha$ stimulated cDNA. Lane 4: BepA1 WT transfected and TNF- $\alpha$ stimulated cDNA. Lane $\boldsymbol{E}$ is an empty lane. Lane 5: FIC domain mutant transfected, not TNF- $\alpha$ stimulated cDNA. Lane 6: FIC domain mutant transfected, TNF- $\alpha$ stimulated cDNA. The ladder (L) used is the $1 \mathrm{~kb}$ Hyperladder from BIOLINE.

NanoDrop quantification indicated that the cDNA was good quality and concentrated, a result of good quality RNA purified from cell lysates. Based on gel analysis and NanoDrop quantification of the cDNA templates, we decided to proceed with PCR.

\subsubsection{PCR of cDNA samples}

After cDNA synthesis we proceeded with semi-quantitative PCR reactions, using the cDNA preparations as template. In total we tested for the expression of five target genes: Cyclophilin A, IL-6, IL-8, SM22 and Myozap, optimising the PCR annealing temperatures for each gene. Each PCR reaction was checked via gel electrophoresis (Figures 4.2, 4.3 and 4.7). We performed PCR on our cDNA samples for two major reasons: firstly, to confirm that our target genes could be detected, indicating that our cDNA templates were of good, workable quality, and secondly to check that our primers allowed specific amplification of the correct targets. Together these allowed us to amplify our target genes in qPCR without any issues about cDNA template quality or amplicon specificity. PCR was also done for the Myozap gene to 
indicate the presence or absence of mRNA transcripts, as changes in expression were not predicted and thus not quantified by qPCR.

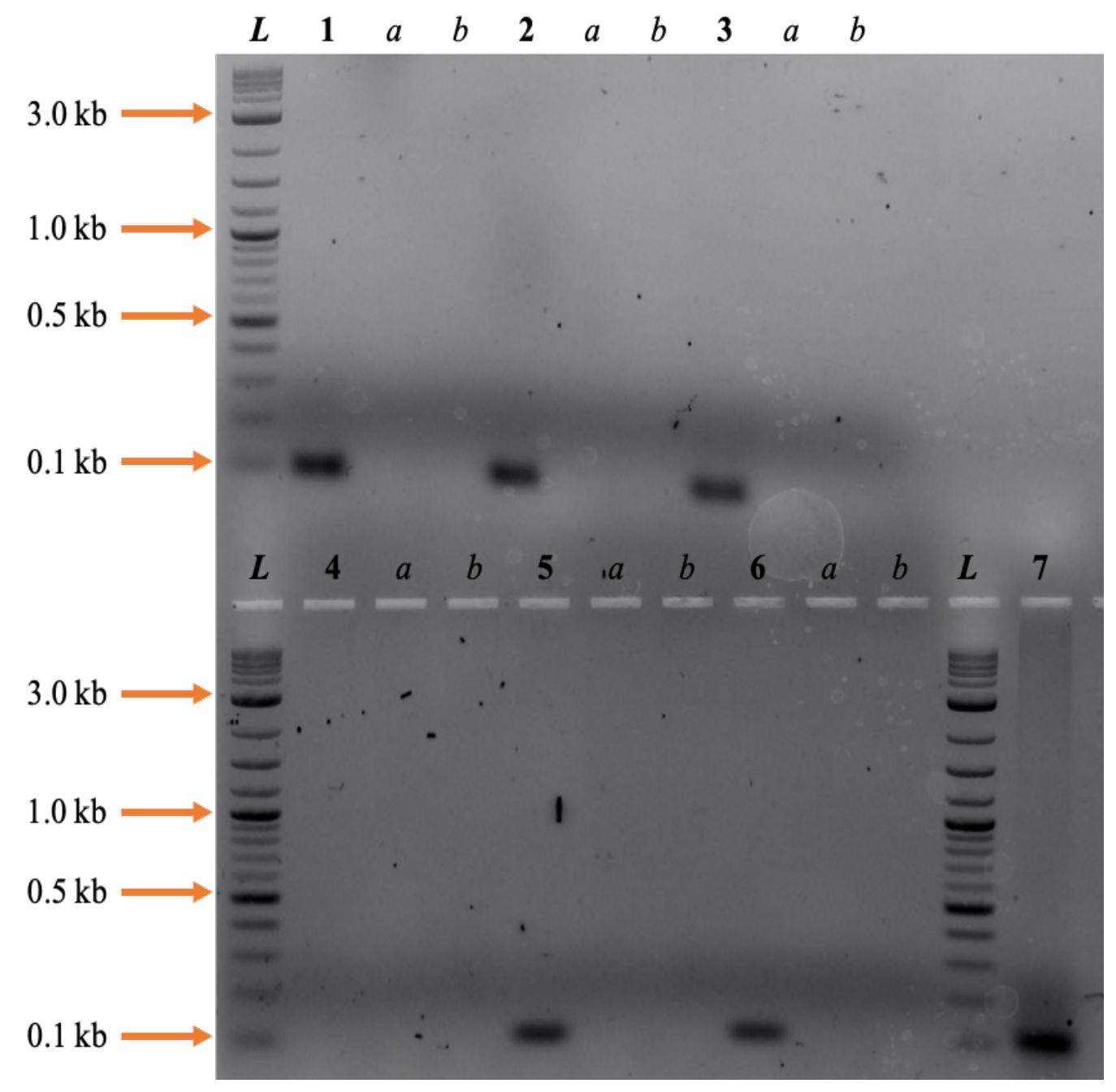

Figure 4.2 Gel analysis of PCR of reference gene, Cyclophilin A. The Cyclophilin A PCR product is predicted to be $98 \mathrm{bp}$, and bands were seen at $\sim 100 \mathrm{bp}$, consistent with this prediction. The gel was loaded as follows: Lane 1: HeLa basal (untransfected, not TNF- $\alpha$ stimulated) sample; Lane 2: HeLa untransfected and TNF- $\alpha$ stimulated sample; Lane 3: BepA1 WT transfected, not TNF- $\alpha$ stimulated sample; Lane 4: BepA1 WT transfected and TNF- $\alpha$ stimulated sample. Lane 5: BepA1 FIC domain mutant transfected, not TNF- $\alpha$ stimulated sample; Lane 6: BepA1 FIC domain mutant transfected, TNF$\alpha$ stimulated sample. For each sample, a represents the No Template Control (NTC) and b represents the no Reverse Transcriptase Control (no RTC). Lane 7: PCR reaction with genomic DNA as template rather than cDNA template. The ladder $(\mathbf{L})$ is the Hyperladder $1 \mathrm{~kb}$ Plus DNA Ladder (BIOLINE). 

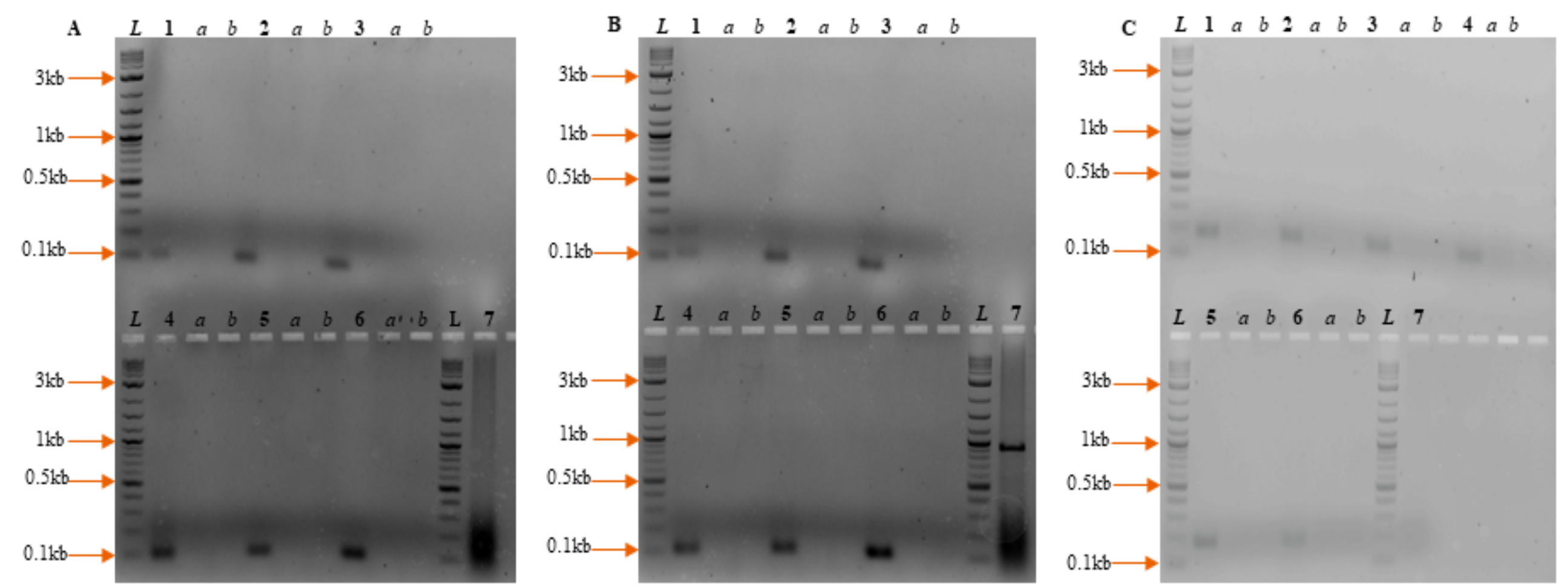

Figure 4.3 Gel analysis of PCR for IL-6, IL-8, and SM22. Each gel represents an optimised PCR amplification of each gene product. Gels are as follows: A is the IL-6 gel, B is the IL-8 gel and $\mathbf{C}$ is the SM22 gel. The respective sizes for each amplified PCR product are as follows: IL-6, 99 bp; IL-8, 107 bp; and SM22, 180 bp.

In the IL-6 and IL-8 gels, the genomic DNA lane (lane 7) produced a large smear, possibly because the amount of template used was too high. In gel B, the genomic DNA control has an additional band near the $1 \mathrm{~kb}$ mark. The samples in the lanes are as follows: Lane 1: HeLa basal (untransfected, no TNF- $\alpha$ treatment) sample; Lane 2: HeLa untransfected and TNF$\alpha$ treated sample; Lane 3: BepA1 WT transfected, no TNF- $\alpha$ treatment sample; Lane 4: BepA1 WT transfected and TNF- $\alpha$ treated sample; Lane 5: BepA1 FIC domain mutant transfected, no TNF- $\alpha$ treatment sample; Lane 6: BepA1 FIC domain mutant transfected, TNF- $\alpha$ stimulated sample. These PCR reactions contains two negative controls: lane a: The No Template Control (NTC) for each sample and lane b: the no Reverse Transcriptase Control (RTC) for each sample. Lane 7: genomic DNA PCR reaction run with genomic DNA as template rather than cDNA template. $\boldsymbol{L}$ represents the ladder used: Hyperladder 1kb Plus DNA Ladder (BIOLINE). 
Each gel shows the specific amplification of our target genes. For Cyclophilin A, numbered lanes 1-3 and lanes 5 and 6 showed a consistent level of Cyclophilin A expression across all samples. Lane 4, however, did not contain product, likely because the reaction did not contain any cDNA template during the set-up of the PCR reaction. The most likely cause of this is a pipetting error, as PCR reaction volumes can be quite small. All other bands in numbered lanes 1-3 and lanes 5 and 6 are at the appropriate size for the Cyclophilin A PCR product gene fragment, $98 \mathrm{bp}$, indicating specific amplification of Cyclophilin A. All control lanes with no template (NTC; all lanes $a$ ) or without reverse transcriptase (no RTC; all lanes $b$ ) contain no product, indicating that these reactions are free from genomic DNA contamination. The positive control genomic DNA sample produced a strong band at approximately $100 \mathrm{bp}$, consistent with the predicted size of the Cyclophilin A PCR fragment.

In the IL-6 gel, the individual product bands are easily visible. While in lane 1 the product is predictably faint (indicating low basal levels of IL-6 in the HeLa basal sample), for lanes 2 - 6 the bands are stronger but not consistent, indicating an increase in IL-6 synthesis across different samples. For IL-8, the individual product bands are also easily visible, with the product band in lane 1 (HeLa basal) appearing notably fainter than the other product bands. Additionally, there is also another second product in lane 1, indicated by the red arrow. This could be due to different splice variants of the same gene or could be a by-product of the lower annealing temperature for the IL-8 PCR reaction. If lower annealing temperature is the cause, then qPCR on IL-8 primer would indicate any non-specific product amplification.

Like the IL-6 gel, the IL-8 products in lanes 2 - 6 are stronger and not consistent in their intensity, indicating to an increase in IL-8 synthesis across these 5 samples. In both IL-6 and IL-8 gels, the genomic DNA sample produced large smears, suggesting that too much template was used in the reaction. For the IL-8 genomic DNA reaction, a band at the $1 \mathrm{~kb}$ mark is also visible, suggesting non-specific product amplification. For both gels, negative controls, NTC or the no $\mathrm{RTC}$, in lanes a and $\mathrm{b}$ respectively, confirm the absence of genomic DNA contamination.

Lastly, SM22, the SRF-regulated gene, is visible in the gel image. The PCR products are faint, suggesting the expression of SM22 in HeLa cells is naturally low, and furthermore, does not seem to be affected by either TNF- $\alpha$ treatment or by BepA1 WT and the FIC domain mutant protein transfection. However, these observations would require further validation by qPCR. Unfortunately, the genomic DNA control did not produce a product in this PCR reaction, possibly because not enough template was used. 
In all of the PCR gel analyses, neither of the two negative controls, NTC or no RTC, produced any visible bands, indicating that our cDNA was free of genomic DNA and that we had no primer-dimer formation. Primer-dimers are potential biproducts of PCR reactions where primers have hybridized to each other and are amplified by the DNA polymerase. These are usually seen as weaker bands that are much smaller than the target products. Absence of these biproducts demonstrates that our primers specifically amplify their target products. While not all our genomic DNA (positive control) samples produced product, amplification of our target products from cDNA templates demonstrated that our PCR reactions had overall worked as expected.

Unfortunately, we could not repeat these PCR experiments to due to time restrictions and were unable to show amplification of Cyclophilin A in the BepA1 WT transfected, no TNF- $\alpha$ sample (Figure 4.2, Lane 4).

\subsection{3 qPCR of IL-6, IL-8 and SM22}

Our RT-PCR reactions demonstrated that our cDNA template was of good quality and that our primers specifically amplified the target genes. We next proceeded with a quantitative analysis of gene expression in our cDNA samples via qPCR. We used qPCR to determine if the levels of IL-6, IL-8 or SM22 changed in response to TNF- $\alpha$ and transfection with the BepA1 WT and the FIC domain mutant. The housekeeping gene Cyclophilin A was included as our reference gene.

Each quantification of our target genes produced an amplification plot and each primer pair was also tested for target specificity via a melt curve. Myozap was not quantitatively assessed as we did not anticipate the expression levels would change in response to transfection.

The results of the qPCR amplification of the reference genes and targets genes are presented in Figure 4.4. 

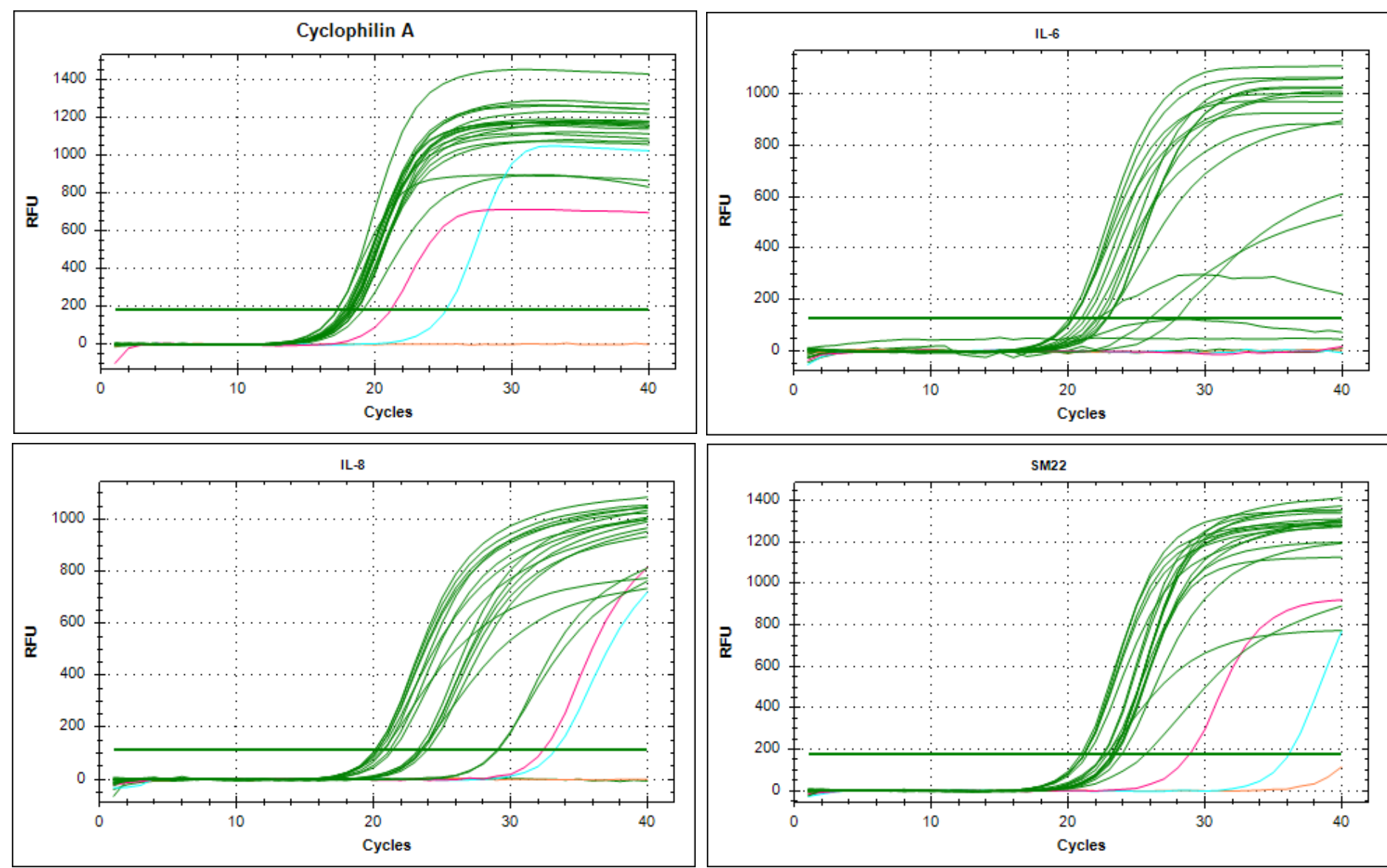

Figure 4.4 Amplification plots for Cyclophilin A, IL-6, IL-8 and SM22. Amplification plots based on the threshold cycles $\left(\mathrm{C}_{\mathrm{T}}\right)$ values are shown for Cyclophilin A, IL-6, IL-8 and SM22 in the qPCR SYBR Green assays using HeLa cell sample cDNA. Green curves represent the target gene amplification in the samples (6 samples, with 3 triplicates each, total 18 samples). The three other coloured lines represent our controls: the negative controls, No Template Control (NTC) and no Reverse Transcriptase Control (RTC), are peach and light blue respectively. The genomic DNA positive control is pink. The solid green horizontal line is the threshold line, representing the level of detection at which the individual reactions have reached a fluorescence intensity above background levels. The threshold line was determined by the qPCR machine software. RFU= Relative Fluorescence Units. 
Amplification plots represent the accumulation of product throughout the duration of the qPCR reaction, where amplification is measured as fluorescence. For the individual amplification plots for Cyclophilin A, the reference gene, fluorescence steadily increased after cycle $\sim 12$ and plateaued at cycle $\sim 22 / 24$. The amplification in our HeLa cell samples (green curves) generally clustered together, with the exception of a couple of samples. This indicates that the gene expression levels of Cyclophilin A in the HeLa cell samples are fairly consistent. This is a good sign as the reference genes expression is not expected to change in the cell type, regardless of the manipulation. Some of the variation may be due to minor pipetting variability during the setup of the $\mathrm{qPCR}$ reaction.

For each qPCR reaction we had three controls: two negative controls, (NTC and no RTC), both lacking a cDNA template, and as a result, not expected to result in target gene amplification. For Cyclophilin A, the NTC (peach line) showed no amplification, indicating there is no contamination in the qPCR reaction mix and primers. The no RTC controls for genomic DNA contamination. In this qPCR reaction, the no RTC (light blue line) control shows amplification of Cyclophilin A, which was an unexpected result and indicates somewhere in the reaction process, the sample became contaminated. In contrast, our positive genomic DNA control (pink line), shows amplification of Cyclophilin A as expected.

Cyclophilin $\mathrm{A}$ is a housekeeping gene performing essential functions in protein folding and trafficking [145]. Expression of a good reference gene should be consistent across all samples regardless of the manipulation, and constitutive housekeeping genes that maintain basic cellular function should meet this criterion [146]. Figure 4.2 shows the gel analysis for PCR of Cyclophilin A from cDNA. Though only semi-quantitative, the consistent level of gene expression in lanes 13 and lanes 5-6 provides confidence in this reference gene. Other common reference genes include $\beta$-actin and GAPDH, however, some reports have indicated that these genes are not consistently expressed across samples and can vary significantly in different cell lines [146].

Together, the qPCR results for our reference gene Cyclophilin A show that untransfected HeLa cells/BepA1 WT and FIC domain mutant transfected HeLa cells under different stimulation conditions (with or without TNF- $\alpha$ ), produce similar levels of Cyclophilin A. While our controls indicate we did have some genomic DNA contamination, we were able to demonstrate a fairly consistent level of Cyclophilin A across HeLa cells samples.

For IL-6, the amplification plot is a little anomalous. For many of the HeLa cell samples, the amplification curves are strange and do not extend beyond the threshold line. These curves indicate that there is still some base level of amplification, but it is not enough to rise above background levels. Furthermore, the genomic DNA control did not show amplification of IL-6, 
where it is expected to. These results suggest a potential problem with the primer specificity, although the amplification of IL-6 from many of the samples suggests the problem is not the primers. Rather, these anomalous results may have been due to pipetting errors introduced during the setup of the qPCR reaction, resulting in inadequate ejection of the qPCR reagents and/or template. Our negative controls, however, show no target gene amplification, indicating no contamination within our reagents or the sample preparation. These results do deem the IL-6 qPCR a little unreliable but due to time constraints, we were not able to repeat this experiment as warranted.

The IL-8 qPCR reaction is better, showing the amplification of IL-8 in all HeLa cell samples (green curves). While the individual amplification curves are not clustered together, as seen in the Cyclophilin A plot, we did not expect to see similar levels of IL-8 expression across these HeLa cell samples. The more dispersed nature of these amplification curves suggests the expression level of IL-8 varies across the samples and indicates that transfection with the BepA1 WT plasmid affects IL-8 expression. In general, there is a steady increase in fluorescence after cycle $\sim 18$, followed by a plateau at cycle $\sim 32$ for most of the samples. Some amplification curves are seen amplifying at cycle $\sim 26$ and do not reach the maximal level of fluorescence intensity as the majority of the HeLa cell samples. This result may suggest a lower IL-8 expression level in these samples, but further experimental validation and analysis is required to demonstrate this.

Our controls for IL-8 indicate potential genomic DNA contamination in our no RTC control, as we see amplification of IL-8 in this sample (light blue curve). The NTC control (peach) shows no target gene amplification, demonstrating that the reagents and primers are free from genomic DNA contamination. The positive genomic DNA control (pink) shows amplification of IL-8 however this was $\sim 10$ cycles later than the HeLa cell samples. This perhaps is due to not spinning down the qPCR plates prior to running the qPCR reaction but repeating these experiments will provide better evidence.

Our last target gene, SM22, shows amplification in our HeLa cell samples (green bars). Most of these curves are clustered together, similar to Cyclophilin A, apart from a couple of curves that were detected with a lower fluorescence intensity. We can see a steady increase in fluorescence after cycle $\sim 18$ which plateaus at cycle $\sim 30$ for most samples; a couple of samples show smaller amplification curves, beginning to amplify SM22 at cycle $\sim 20$ but do not reach the maximal fluorescence intensity as the majority of other HeLa cell samples. These results could suggest a lower level of SM22 in these samples, and the clustering of these samples suggests that expression of SM22 may not significantly differ between the samples. Statistical analysis, however, will shed further light on the gene expression changes. 
Our negative control, without any added template (peach curve) shows no amplification of the SM22 target. However, there is a noticeable increase in fluorescence intensity towards cycle 38. Amplification after this many cycles is not likely to be an amplification of the target gene. Our second negative control, the no RTC control (blue curve) shows amplification of SM22, also indicating genomic DNA contamination here. The positive genomic DNA control (pink curve) shows amplification of the SM22 target, but this was later than the HeLa cell samples, where a fluorescence signal was detected at cycle $\sim 16$.

In all our qPCR experiments, there was at least one element that prevents these results from being entirely trustworthy. This includes potential genomic DNA contamination or pipetting errors that have resulted in strange amplification curves. Due to these factors, further experimental validation is required for each target gene. Unfortunately, time pressures made further repeats impossible and so only one biological replicate of each qPCR reaction was performed. Our qPCR experiments are preliminary experiments that have not been optimised. However, these results present some very intriguing pilot data suggesting that IL-6 and IL-8 expression is depressed in HeLa cells in the presence of the WT BepA1 protein, relative to the FIC domain mutant. The next step will be to further validate these observations.

After we had performed the qPCR reactions with our target genes, we next conducted a melt curve analysis on each target gene primer pair. A melt curve analysis is used to determine that each primer pair is specific for its target product. The process involves heating the reaction mixture at the end of the qPCR reaction from $65^{\circ} \mathrm{C}$ to $90^{\circ} \mathrm{C}$ in increments of $0.5^{\circ} \mathrm{C}$. The resulting output is a melt peak that shows the dissociation of the primers from the target product. An ideal melt peak shows one very distinct peak for each primer pair which allows us to determine two things: first that the primers are selectively amplifying a single discrete species of DNA and secondly, that there are not formations of primer dimers in the reaction (seen as an extra peak). As our qPCR experiments used SYBR green fluorescent dye, a non-specific method of quantitative amplification, it became important to further show our primers are specific for their intended target gene and do not bind to each other, resulting in dimer formation. As we had performed PCR on our cDNA prior to qPCR, and the gel analyses showed that we had specific amplification of our target genes, we expected to see only one distinct peak in the melt curve for each primer pair.

The individual melt peaks are shown in Figure 4.5 below, with the curves for the controls coloured to aid better visualisation. 

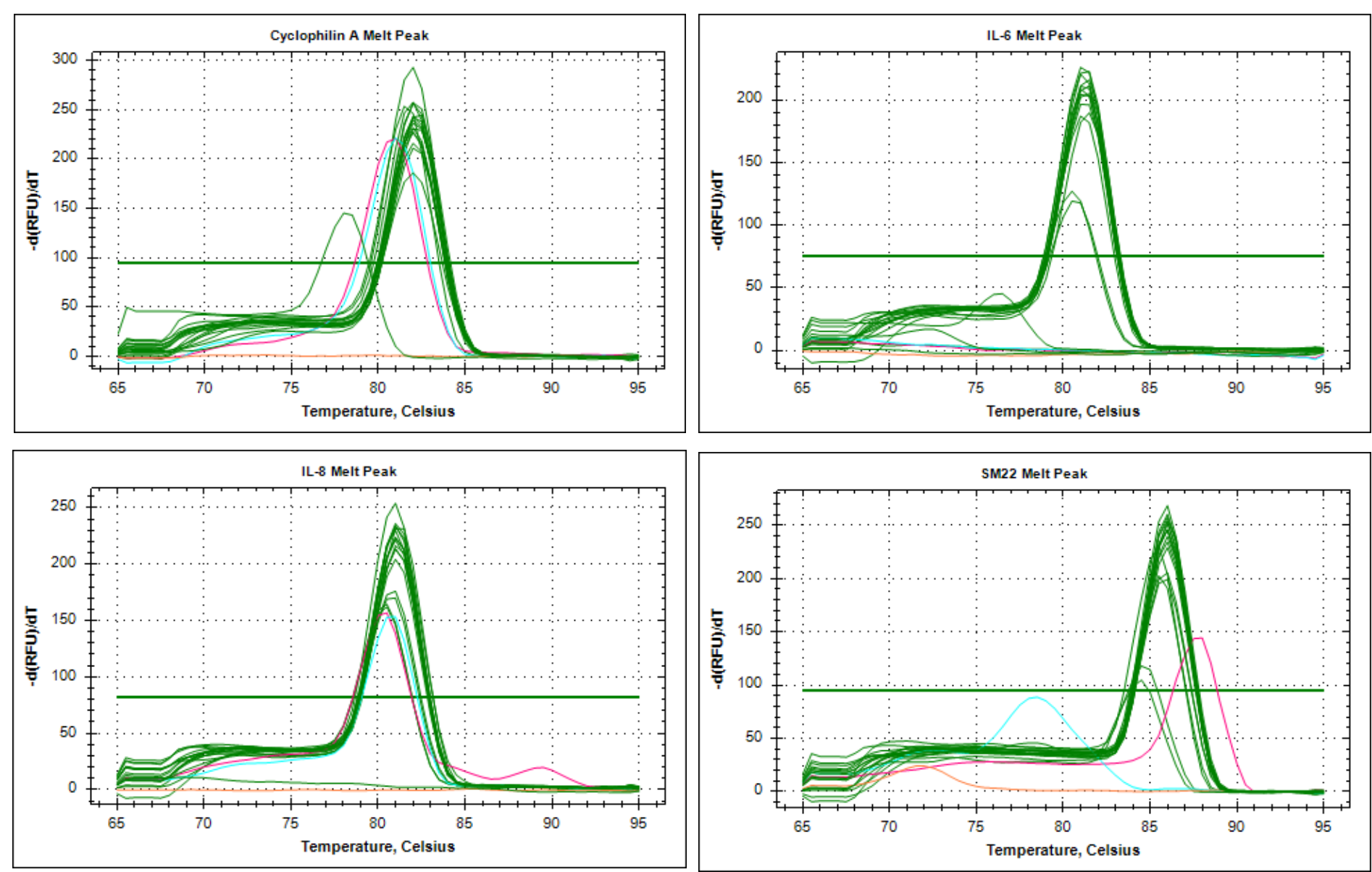

Figure 4.5 Melt peaks for all primers used in qPCR. For Cyclophilin A, the melt range is from $78.0^{\circ} \mathrm{C}-82.0^{\circ} \mathrm{C}$; for IL-6; for IL-8, the melt range is from $80.5^{\circ} \mathrm{C}-81.5^{\circ} \mathrm{C}$ and for SM22 the melt range is from $84.5^{\circ} \mathrm{C}-86^{\circ} \mathrm{C}$. None of the primers in the NTC control (peach coloured curve) produced a melt peak. For IL-6, none of the three controls produced a melt curve. For SM22, neither the NTC control nor the no RTC control produced a melt curve. The controls are the coloured curves: the NTC (no template control) is peach, the no RTC (no reverse transcriptase control) is light blue, and the genomic DNA control is pink. $\mathbf{R F U}=$ Relative Fluorescence Units. $-\mathbf{d}(\mathbf{R F U}) / \mathbf{d T}=$ change in $\mathbf{R F U} /$ change in temperature. 
For each of the 4 graphs presented, there is one very distinct peak for each primer pair with the majority of our HeLa cell samples (green bars). Also noticeable are peaks that are separate from the main melt peak in each target gene. Often, melt peaks should only show one distinct peak for a target gene in all samples. This single melt peak shows that the amplified DNA is a single discrete species. When multiple peaks are present this can indicate that there has been off target amplification or the presence of contaminating products. However, there are a couple of factors outside of primer specificity that can affect the melt peak analysis. Firstly, primers with a higher GC content can exhibit higher melting temperatures due to stability of the GC bonds. Conversely, primers with a low GC content (high AT content) will dissociate at a lower temperature.

Secondly, DNA dissociation is not always biphasic and can dissociate at multiple temperatures, giving rise to multiple peaks in the melt peak analysis. Additionally, these extra peaks could also be instrument artefacts. As we were only able to perform the qPCR reactions once for each target gene, we could not repeat the experiments to further optimize the reaction. However, prior to qPCR we performed reverse transcriptase PCR with our primers and all these reactions produced one specific product. Of note, there is the gel for IL-8 that shows the amplification of a larger $\sim 1 \mathrm{~kb}$ product in lane 7 but the melt peak analysis did not show additional peaks to suggest off target amplification. The secondary product in the IL-8 gel is likely due to the lower annealing temperature used in the reverse transcriptase PCR experiment.

To calculate gene expression changes from the amplification plots, we conducted analysis using the $2^{-\Delta \Delta \mathrm{C} q}$, or Livak, method. The Livak method calculates a normalised expression ratio that represents the fold change - fold increase or decrease - of the target gene in comparison to the reference gene and the calibrator sample. The Livak method assumes that both target and reference genes are amplified at efficiencies near $100 \%$ of each other and within $5 \%$ of each other. This method involves first normalising the expression of the target gene to the reference, Cyclophilin A and then comparing the expression of the target gene to the calibrator sample. Our calibrator sample was the untransfected HeLa cells not treated with TNF- $\alpha$. For the qPCR reaction, each sample was tested in triplicate, resulting in three individual $\mathrm{C}_{\mathrm{T}}$ values for each sample. Using this data, we calculated the normalised expression ratio of each sample. The results are presented in Figure 4.6 below. 


\section{IL-6}

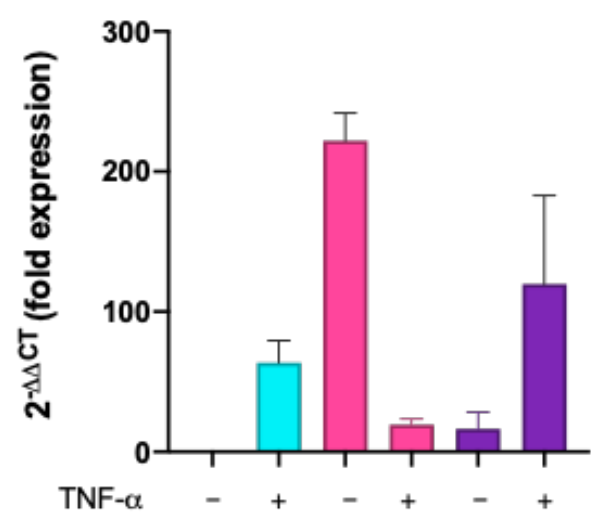

SM-22

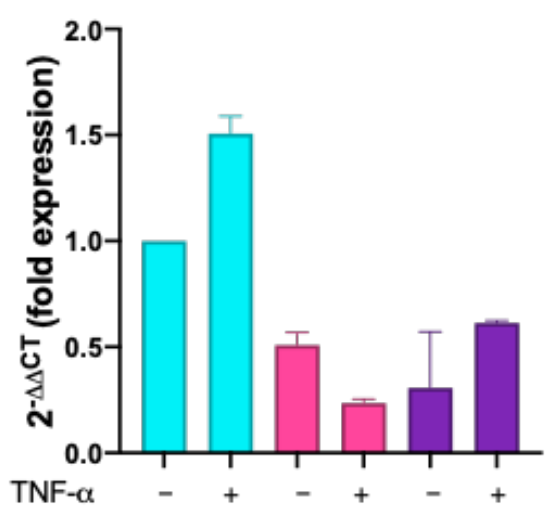

\section{IL-8}

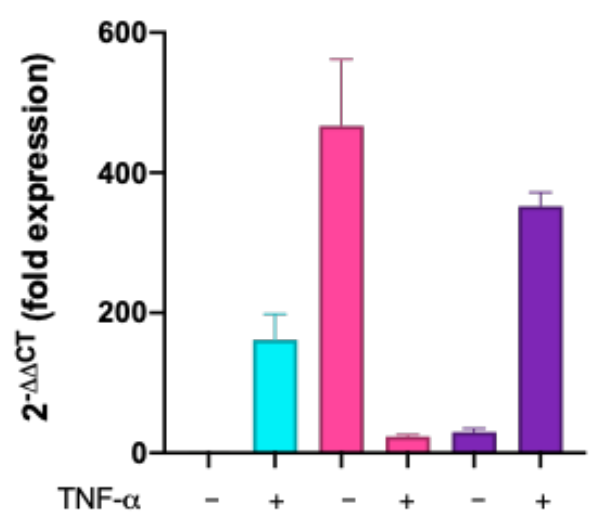

HeLa cells (untransfected)

BepA1 wild type transfected

BepA1 FIC mutant transfected

Figure 4.6 Normalised expression ratios for target genes IL-6, IL-8 and SM22 using the qPCR data. Relative expression changes for IL-6, IL-8 and SM22 were calculated using the Livak method. The reference gene was a housekeeping gene $(\mathrm{cyclA})$ and samples were normalised against untransfected, unstimulated HeLa cells. The individual $\mathrm{C}_{\mathrm{q}}$ 's of the target gene were compared to the reference gene (using an average) and normalised against the $\Delta \mathrm{C}_{\mathrm{q}}$ of the calibrator.

The calculated gene expression ratios show some interesting results. The overall profile of IL-6 and IL-8 were very similar between the samples tested. For both of these, HeLa cells transfected with BepA1 WT, with no TNF- $\alpha$ stimulation, show a large increase in the expression. This was a very surprising result and not what we expected. The gene expression ratios of cells transfected with BepA1 WT are much higher compared to those of cells transfected with the BepA1 FIC domain mutant, without TNF- $\alpha$. This trend is not seen with SM22.

These results are not due to any differences in HeLa cell number between samples or difference in the concentrations of the BepA1 WT and the FIC domain mutant plasmids introduced into these cells. Cells were seeded identically to ensure the same cell number is present in each 
samples and cells were always at $75-80 \%$ confluency. Furthermore, both plasmids were also purified to the same concentrations prior to transfecting.

The introduction of foreign DNA into a cell results in activation of DNA sensors - DNA PRRs, stimulating the cell's innate immune response, so this could be responsible for some of the unexpected results [147]. Moreover, as cytokine and chemokine signalling networks are so complex, we cannot exclude the possibility that internal signalling within or between different pathways may have altered IL-6 and IL-8 expression. For example, the chemokine MIF can stimulate the release of multiple pro-inflammatory cytokines, including IL-6 and IL-8. There could be crosstalk between signalling pathways that were not factored in. TNF- $\alpha$ also stimulates IL-6 and IL-8 secretion, but oddly, we see gene expression levels of these cytokines decrease in BepA1 WT transfected cells stimulated with TNF- $\alpha$.

SM22 gene expression appears marginally affected by the presence of the BepA1 FIC domain, though there are lower expression levels in the transfected cells relative to untransfected. This suggests the regulatory factors of this gene are not targets of the BepA1 WT protein. SM22 expression also is not particularly affected by the presence of TNF- $\alpha$. Figure 4.6 shows that the changes in SM22 gene expression were overall very minor. Please see appendix figures 7.6.1 7.6.3 for the raw data and Livak calculations for the qPCR data for IL-6, I1-8 and SM22.

\subsubsection{Confirmation of expression of Myozap}

In order to determine whether the gene encoding Myozap is transcribed in HeLa cells, we carried out reverse transcriptase PCR on cDNA derived from HeLa cell samples. Since we were asking about presence or absence, and changes in gene expression were not expected, RT-qPCR was not carried out for this gene. The gel analysis of the PCR reactions with primers amplifying Myozap shows that HeLa cells do transcribe the Myozap gene (Figure 4.7). However, the individual product bands are difficult to see because they are faint, but also because of the UV shadow from the loading dye. Thus, the Myozap products, predicted to be $106 \mathrm{bp}$ in size, are indicated by the red arrows. Judging from the gel image alone, it appears that expression of Myozap between the six HeLa cell samples does not vary radically, although qPCR would be needed to confirm this. The genomic DNA control produced a strong product at the predicted size though there is a moderate smearing, indicating that too much genomic DNA template was used. Our negative controls, which lack any added template (NTC) or cDNA samples that were made without any added reverse transcriptase (no RTC) are shown in lanes a and b respectively for each 
sample. No product amplification is seen for any control, indicating there was no detectable genomic DNA contamination in the samples.

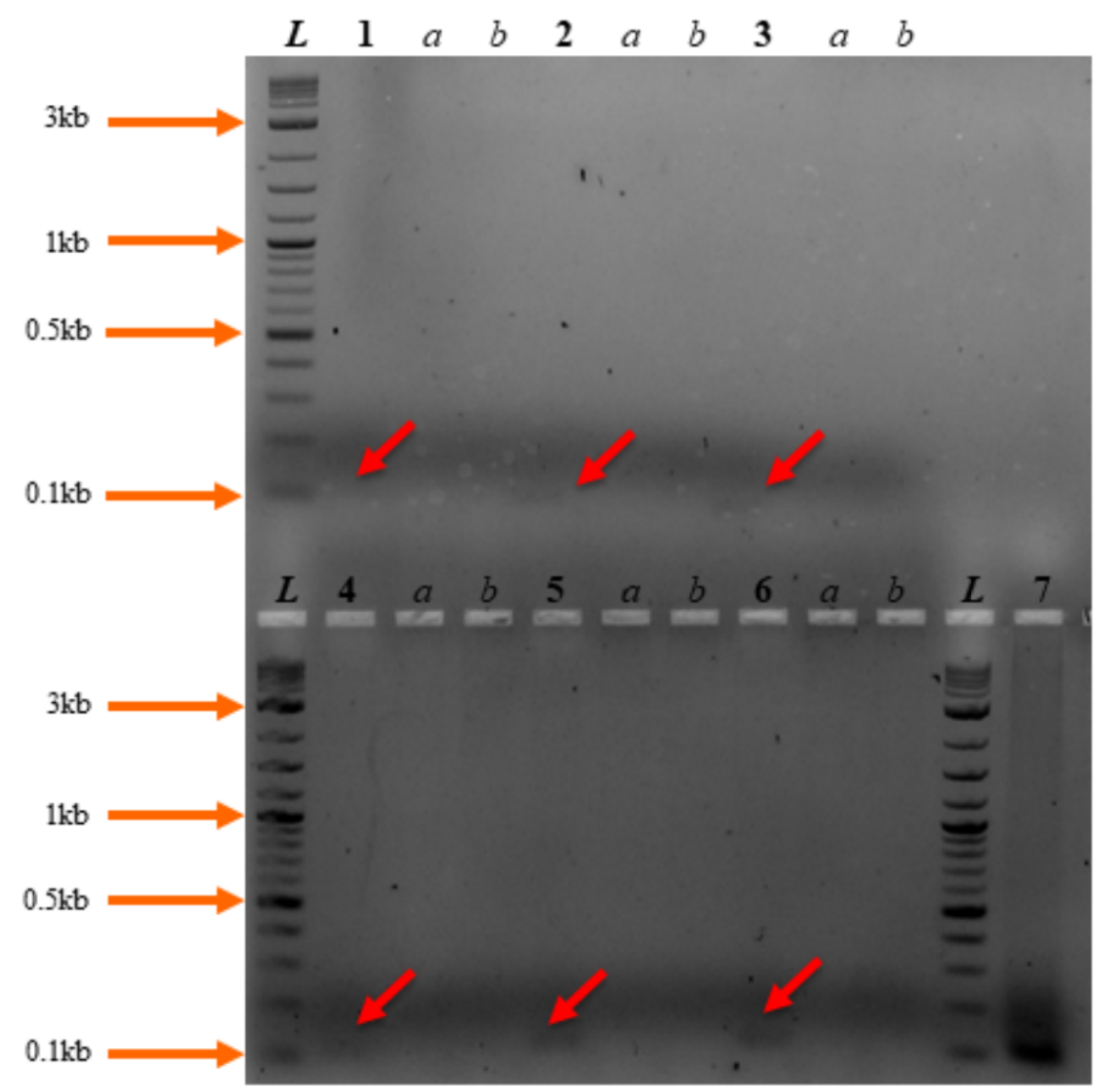

Figure 4.7 Gel analysis of Myozap. The predicted Myozap gene PCR product is $\mathbf{1 0 6} \mathrm{bp}$ in size and the presence of bands at $\sim 100$ bp suggests the Myozap transcript was present in the RNA samples used in generating the cDNA template. The samples in the lanes are as follows: Lane 1: HeLa basal (untransfected, no TNF- $\alpha$ treatment) sample; Lane 2: HeLa untransfected, TNF- $\alpha$-stimulated sample; Lane 3: BepA1 WT transfected, no TNF- $\alpha$ treatment sample; Lane 4: BepA1 WT transfected, TNF- $\alpha$ stimulated sample; Lane 5: BepA1 FIC domain mutant transfected, no TNF- $\alpha$ treatment sample; Lane 6: BepA1 FIC domain mutant transfected, TNF- $\alpha$ stimulated sample; a represents the No Template Control (NTC) for each sample and b represents the no Reverse Transcriptase Control (no RTC) for each sample. Lane 7: PCR reaction run with genomic DNA as template rather than cDNA template. The ladder $(\boldsymbol{L})$ used is the Hyperladder $1 \mathrm{~kb}$ Plus DNA Ladder (BIOLINE). 


\subsection{Discussion}

The qPCR experiments presented here have shown some interesting preliminary results. If we look at samples where TNF- $\alpha$ is added we see results somewhat consistent with that the Human Cytokine Array assay has shown - IL-6 and IL-8 expression is decreased in cells transfected with BepA1. However, it is not possible for us to conclude that the WT BepA1 protein is a direct cause of this. It is intriguing that the addition of TNF- $\alpha$ shows a decrease in IL-6 and IL-8 expression and we yet do not have answers for why this happen, nor why IL-6 and IL-8 expression is so high without it, because we did not do the Proteome Profiler array experiment on transfected but non-TNF- $\alpha$-stimulated samples. We do, however, see that the FIC domain mutant transfected HeLa cells fail to show this depression in IL-6 and IL-8 expression suggesting that the functional FIC domain is important.

SM22 gene expression was largely unaffected either by the presence of the BepA1 protein or by TNF- $\alpha$. Together, this suggests that the regulatory factors that control SM22 expression are not impacted by BepA1 or by TNF- $\alpha$. SM22 is a canonical gene controlled by the serum response transcription factor, and our results suggest that BepA1 does not alter that pathway. However, additional experiments are needed to show this definitively.

In order to clearly determine whether BepA1 is exerting an effect on IL-6 and IL-8 gene expression, these qPCR experiments should be repeated, with additional biological replicates, careful handling and pipetting of reagents, and spinning down the prepared qPCR plates prior to running the reaction. Additionally, carrying out both technical and biological replicates will dampen some of the experimental noise, enabling the reliable calculation of gene expression changes.

Additionally, the presence of Myozap cDNA in HeLa cells suggests further experiments can be done to explore the hypothesised BepA1-Myozap interaction. Protein validation studies can be used to explore this interaction. These could include coimmunoprecipitation of tagged BepA1 or Myozap. AMPylation activity of BepA1 could be confirmed by mass spectrometry, e.g., MALDI-TOF analysis of post-translational modifications, including AMPylation, that occur in transfected or infected cells. These studies have been used previously to identify the target protein, and even the AMPylated residue [148$150]$. 


\section{Chapter 5 - Construction of a BepA1_BepA2 B. quintana deletion mutant}

\section{$5.1 \quad$ Introduction}

B. quintana is one of the three clinically relevant Bartonella species to cause significant disease in humans. Of these three species, B. quintana and B. henselae are the most similar at the genomic level. Genomic analyses have revealed that the B. quintana genome is derived from a larger B. henselae-like ancestral genome [151]. Accordingly, B. quintana harbours a circular chromosome of approximately $1,520 \mathrm{kbp}$ whereas $B$. henselae's genome is larger at about 1,900 kbp. Both B. quintana and B. henselae share many similarities but functions of many $B$. quintana virulence proteins remain understudied in comparison to $B$. henselae.

Several B. quintana clinical isolates have had their genomes sequenced, but only the reference strain, B. quintana Toulouse, is annotated. At the time of the Toulouse strain genome annotation, there was confusion surrounding the BepA gene as it contains an internal stop codon which splits the gene into two open reading frames, BepA1 and BepA2. This internal stop codon was assumed to be a point mutation and both genes were annotated as a single pseudogene in the B. quintana Toulouse strain [151]. However, analysis of additional strains has shown that the configuration of two open reading frames, BepA1 and BepA2, is conserved in multiple B. quintana isolates [92].

BepA1 contains the FIC domain, while BepA2 encodes a BID domain and type 4 secretion signal, allowing it to be translocated into host cells. These two proteins may complex together for secretion, but this has not been shown experimentally. Bartonella species that possess a Type IV secretion system have variable numbers of secreted Bep effector proteins, which also vary in terms of their function. B. quintana has six Beps: BepA1, BepA2, BepC, BepE, BepF1 and BepF2. The genetic layout of the Bartonella effector genes in the B. quintana Toulouse strain is shown in Figure 5.1 below. In the schematic of Bartonella T4SS structural and effector genes, the VirB4 and VirD4 loci are shown, as well as the six secreted effector proteins, including their FIC and/or BID domains. BepA1 and BepA2 are separated by a short noncoding region. BepA1 contains the FIC domain (purple rectangle) while BepA2 contains the BID domain (pale green rectangle). 


\section{FIC domain}

\section{BID domain}

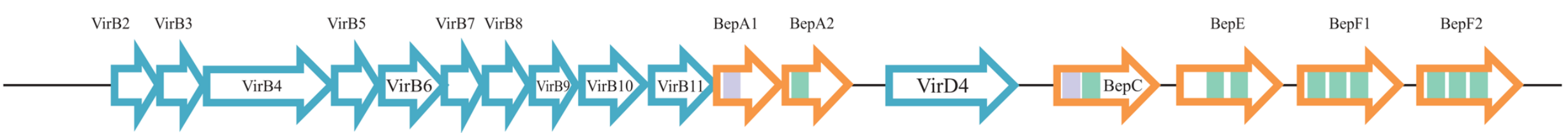

Figure 5.1 B. quintana str. Toulouse T4SS structural and effector genes. The schematic displays the genetic organisation of the T4 secretion structural and effector genes in B. quintana. The six Bep proteins are located downstream of the VirB4 locus, while the VirD4 locus sits between the BepA2 and BepC loci. The FIC and/or BID domains of each effector protein are shown as the purple or green rectangles within the orange arrows, respectively. Adapted from Lambert, C. 2018, from a figure titled "Bartonella Effector genes are semi-conserved between B. henselae and B. quintana" [113]. 
We used the annotated B. quintana Toulouse strain genome sequence to design primers for the construction of a mutagenic plasmid for BepA1 and BepA2. The coding regions of BepA1 and BepA2 are shown in Figure 5.2. The FIC domain of BepA1 is indicated as well. 


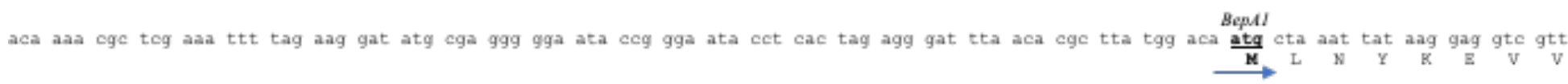

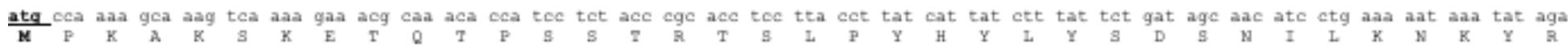

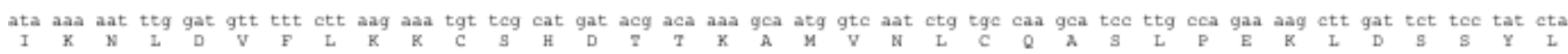

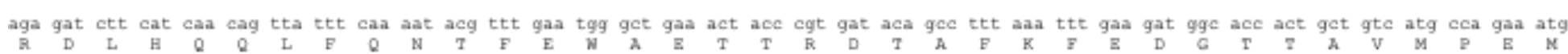

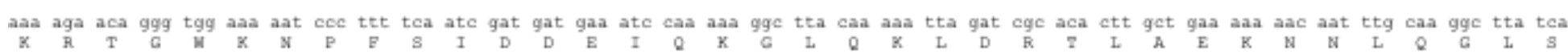

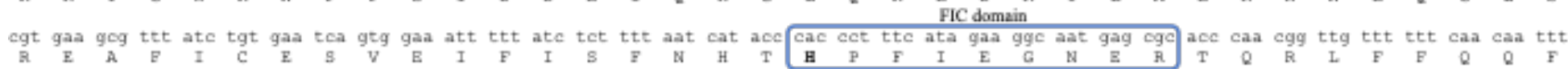

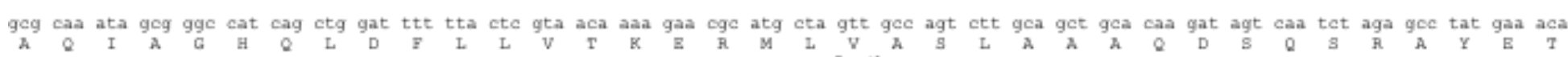

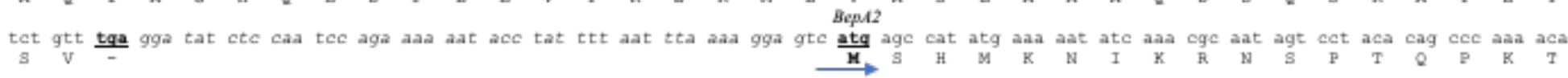

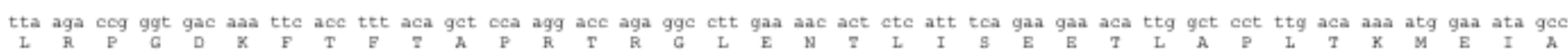

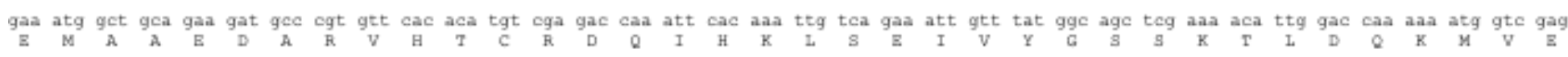

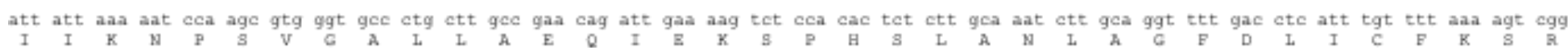

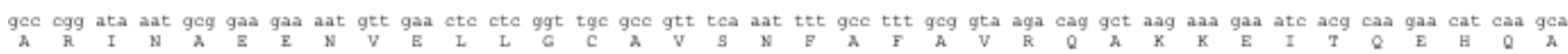

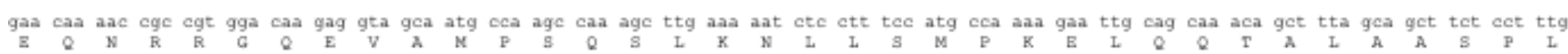
ctt caa aaa gag ctc gaa agt ctt ata aaa caa gtc agt aac cgt ctc tea gga agc gag caa aga goc ctc aag aac aag gat tat gat gca ctc gtg aaa agt ctt

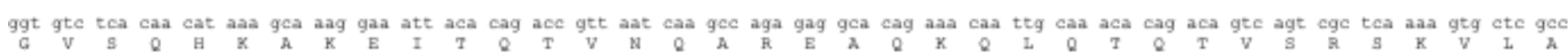

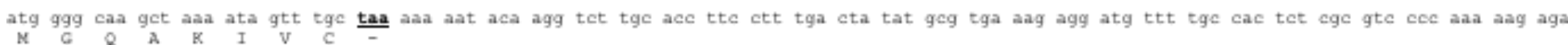

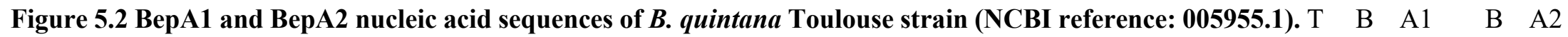

$\mathrm{T}$

$\mathrm{T}$
. $\mathrm{T}$

ATG
ATG

TAA
. $\mathrm{T}$

T FIC
B. quintana 
In this chapter, we aimed to generate a BepA1_BepA2 B. quintana deletion mutant so that we could establish an infection model in a cell culture line, to enable study of host pathogens interactions in vitro. A two-step SacB mutagenesis strategy was used to generate a BepA1 and BepA2 deletion mutant [52].

The SacB mutagenesis strategy involves introduction of the plasmid into B. quintana by bacterial conjugation from $E$. coli $\mathrm{S} 17-1$. The first positive selection is for B. quintana JK31 colonies with the BepA1_BepA2 mutagenesis plasmid integrated into the chromosome. This selection would be followed by a counterselection, for B. quintana JK-31 colonies that have lost the integrated plasmid; counterselection is possible because the plasmid-encoded $s a c B$ gene is lethal in the presence of sucrose. The $s a c B$ gene encodes levansucrase, an enzyme involved in hydrolysis of sucrose and biosynthesis of levan, which cannot be metabolised by the majority of gram-negative organisms, thus rendering it lethal [152]. The mutagenesis strategy is summarised in Figure 5.3 below. The possibility of obtaining B. quintana WT or the deletion mutant from the sucrose selection is likely 50\%; about half of the colonies that lose the plasmid also lose adjacent genome sequence, generating a mutant, while the other half revert to the wild type. 


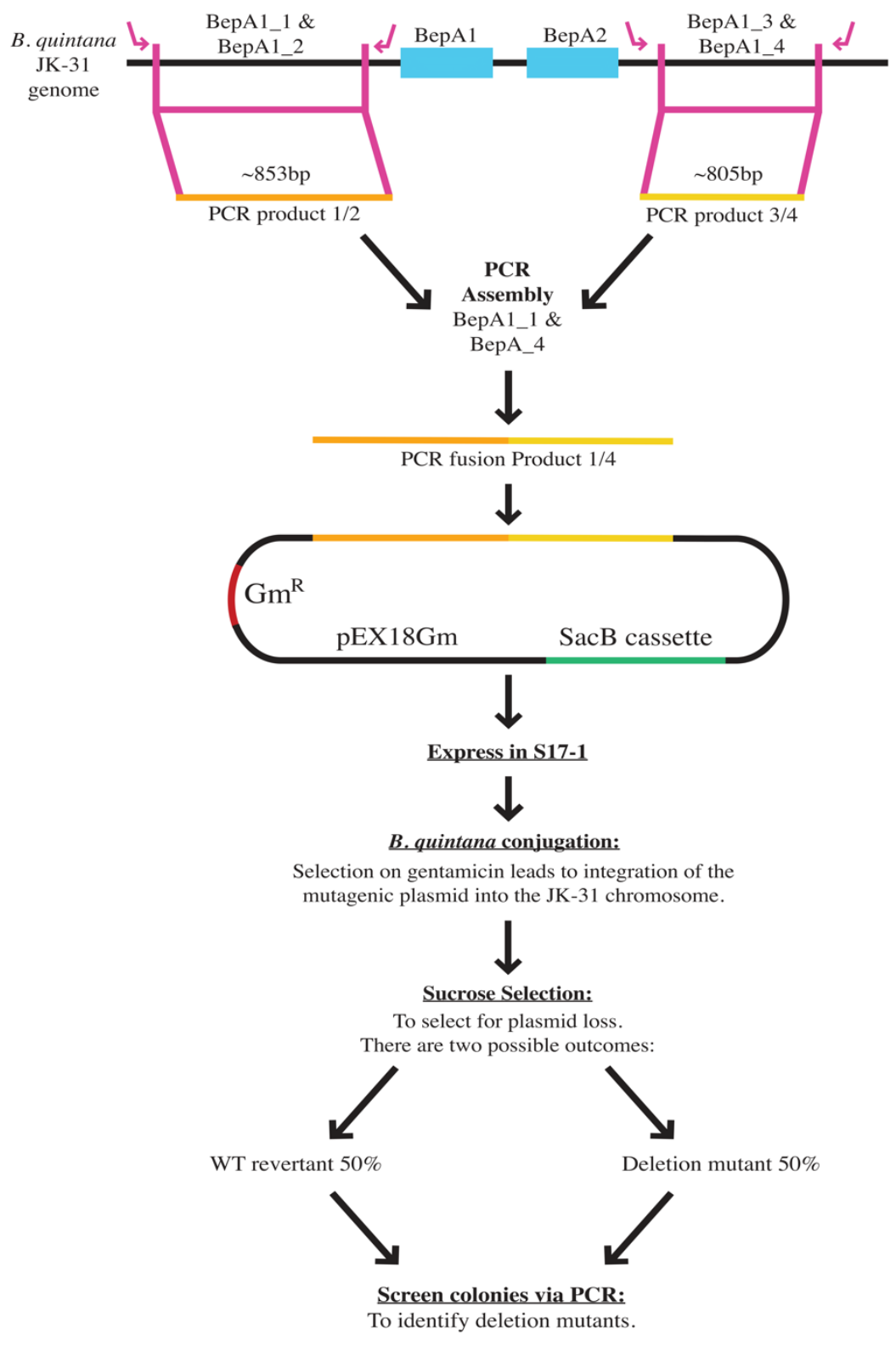

Figure 5.3 The SacB mutagenesis strategy used to construct the BepA1_BepA2 B. quintana deletion mutant. The diagram depicts the strategy we utilised to generate the B. quintana BepA1_BepA2 deletion mutant. First, approximately 800 bp regions immediately surrounding the BepA1 and BepA2 genes were PCR amplified from B. quintana JK31 using primer pairs BepA1_1 and BepA1_2, and BepA1_3 and BepA1_4 respectively. The resultant two PCR products were joined via PCR fusion using primers BepA1_1 and BepA1_4, generating the PCR fusion product $1 / 4$. This fusion product was cloned into $\mathrm{pEX} 18 \mathrm{Gm}$, which is a suicide vector in B. quintana and has gentamicin resistance (red) and a SacB cassette (green), which allows for sucrose counterselection. The BepA1_BepA2 mutagenesis plasmid would be introduced into $B$. quintana JK-31 strain through bacterial conjugation followed by selection on gentamicin. Only JK-31 colonies that have the plasmid integrated onto the chromosome via homologous recombination would be resistant to gentamicin. Colonies with the integrated plasmid would then be grown on sucrose plates, to select for a second round of homologous recombination that results in loss of the plasmid. Two outcomes are possible following the second recombination: reversion to the wild type, or deletion of BepA1 and BepA2. Colonies would be screened by PCR to distinguish between wild type and mutant. 
Conjugation is a tool used in a research lab setting to introduce new DNA into a recipient bacterium and has been frequently used in the literature, with many protocols having been described. Conjugation is a rare event and obtaining transconjugants is difficult with a fastidious organism like $B$. quintana. Therefore, before conducting conjugations with $B$. quintana, we conducted a control experiment to see if the mutagenesis plasmid could be mobilised using E. coli as the recipient. Accordingly, we conducted a test conjugation with our donor strain, E. coli S17-1 carrying our mutagenesis plasmid, a derivative of pEX18Gm, and the recipient, E. coli carrying the plasmid pACYC184, which is compatible with pEX18Gm. Furthermore, pACYC184 confers resistance to chloramphenicol, enabling us to select transconjugants on chloramphenicol and gentamicin. Thus, we could easily distinguish between donor, recipient and transconjugants.

B. quintana is not well-studied, and the functions of each Bep effector protein have not been elucidated, leaving a large gap in our understanding of $B$. quintana pathogenesis. Previous data suggested an immunosuppressive role for the BepA1 protein of B. quintana, with statistically significant reduced expression of IL-6 and IL-8 in lysates of BepA1 WT transfected and TNF- $\alpha$ stimulated HeLa cells, relative to cells transfected with the FIC mutant [113]. Furthermore, BepA2 has been shown to confer anti-apoptotic activity [92]. With our preliminary results, we wanted to investigate the roles of BepA1 and BepA2 in host-pathogen interactions.

Through the generation of our deletion mutant, our aim was to determine the impact of BepA1 and BepA2 in the reservoir human host and their role in bacterial survival and persistence. This knowledge would be invaluable in combating B. quintana infections, especially now that this pathogen is re-emerging in vulnerable populations. 


\subsection{Results}

\subsubsection{Generation of the BepA1_BepA2 mutagenesis plasmid}

To generate a BepA1 and BepA2 deletion mutant, we first constructed a plasmid for mutagenesis. To do this, the immediate surrounding regions of the BepA1 and BepA2 genes were amplified from $B$. quintana JK-31 by PCR. Using primers specifically designed to amplify these flanking regions, we generated two preliminary PCR products. To see how these primers, anneal to the B. quintana JK-31 genome, please refer to Figure 5.3. The primers used to generate these preliminary PCR products are named BepA1_1 and BepA1_2 for the region upstream of BepA1, and BepA1_3 and BepA1_4 for the region downstream of BepA2. The preliminary PCR products can be seen in Figure 5.4. The BepA1 PCR product $1 / 2$ is approximately $853 \mathrm{bp}$ and BepA2 PCR product $3 / 4$ is $805 \mathrm{bp}$.

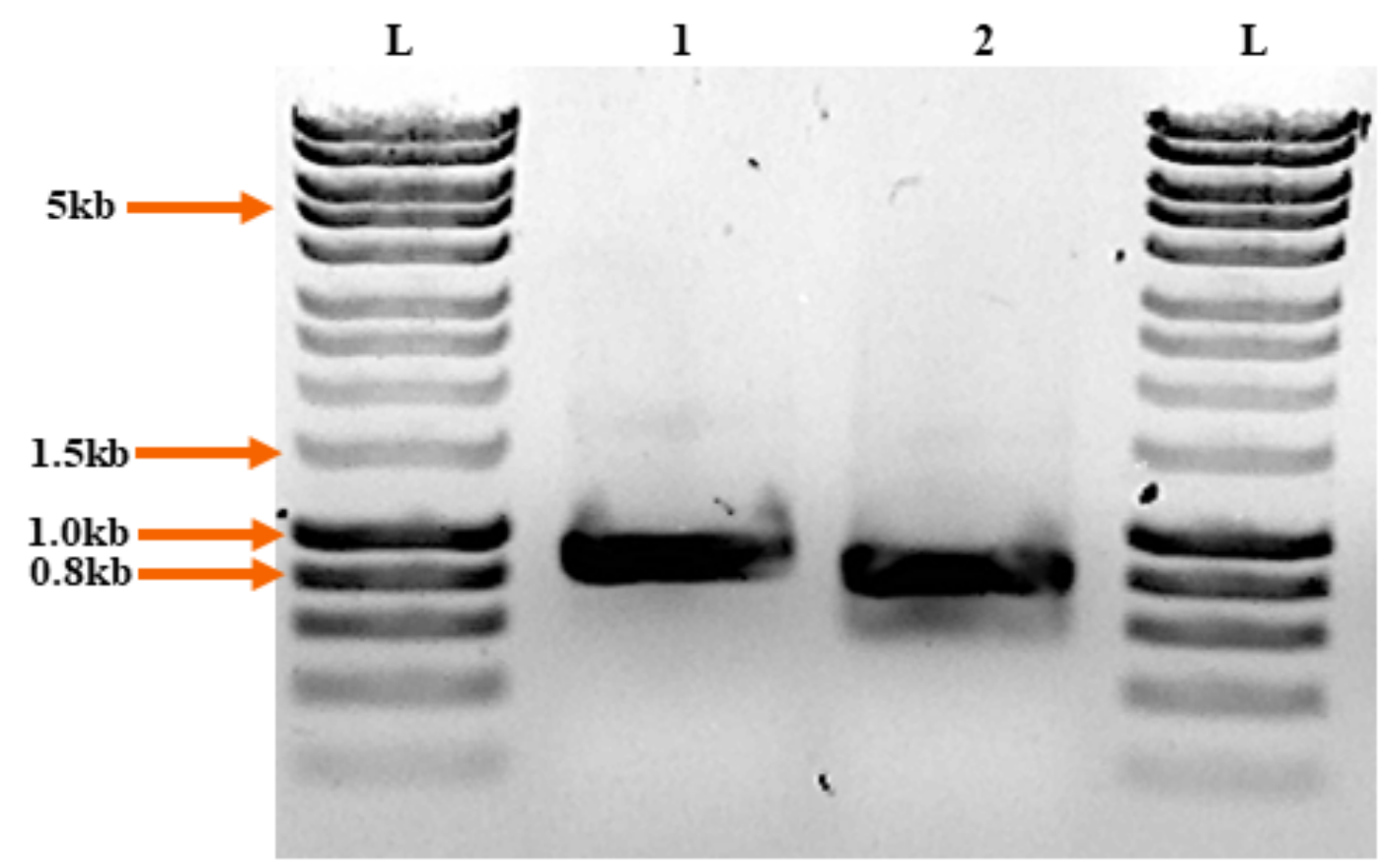

Figure 5.4 PCR products of sequences surrounding BepA1 and BepA2. Samples were run on a 1\% agarose gel. BepA1 PCR product 1/2 (Lane 1) is approximately 853 bp while BepA2 PCR product 3/4 (Lane 2) is $805 \mathrm{bp}$. The ladder (L) is the Hyperladder $1 \mathrm{~kb}$ (BIOLINE).

We opted to amplify $\sim 800 \mathrm{bp}$ regions for the preliminary PCR products to ensure a long enough sequence was available to enable homologous recombination to occur in B. quintana. There is no published data about the optimal length of the homologous sequence in B. quintana to allow homologous recombination, but $800-1000$ bp of sequence is commonly used [59, $118]$. 
Our preliminary PCR products were purified and combined to generate a PCR fusion product. The primers BepA1_2 and BepA1_3 were engineered to have an overlapping sequence. This allowed the two PCR products to self-prime internally and generate the PCR fusion product when combined with the outermost primers, BepA1_1 and BepA1_4, both of which had engineered BgIII restriction sites. Digestion with BglII would allow us to later clone the PCR fusion product 1/4 into a BamHI digested vector.

Using primers BepA1_1 and BepA1_4 we generated the BepA1_BepA2 PCR fusion product. The total length of this product was predicted to be $1,658 \mathrm{bp}$. The purified PCR fusion product is shown in Figure 5.5.

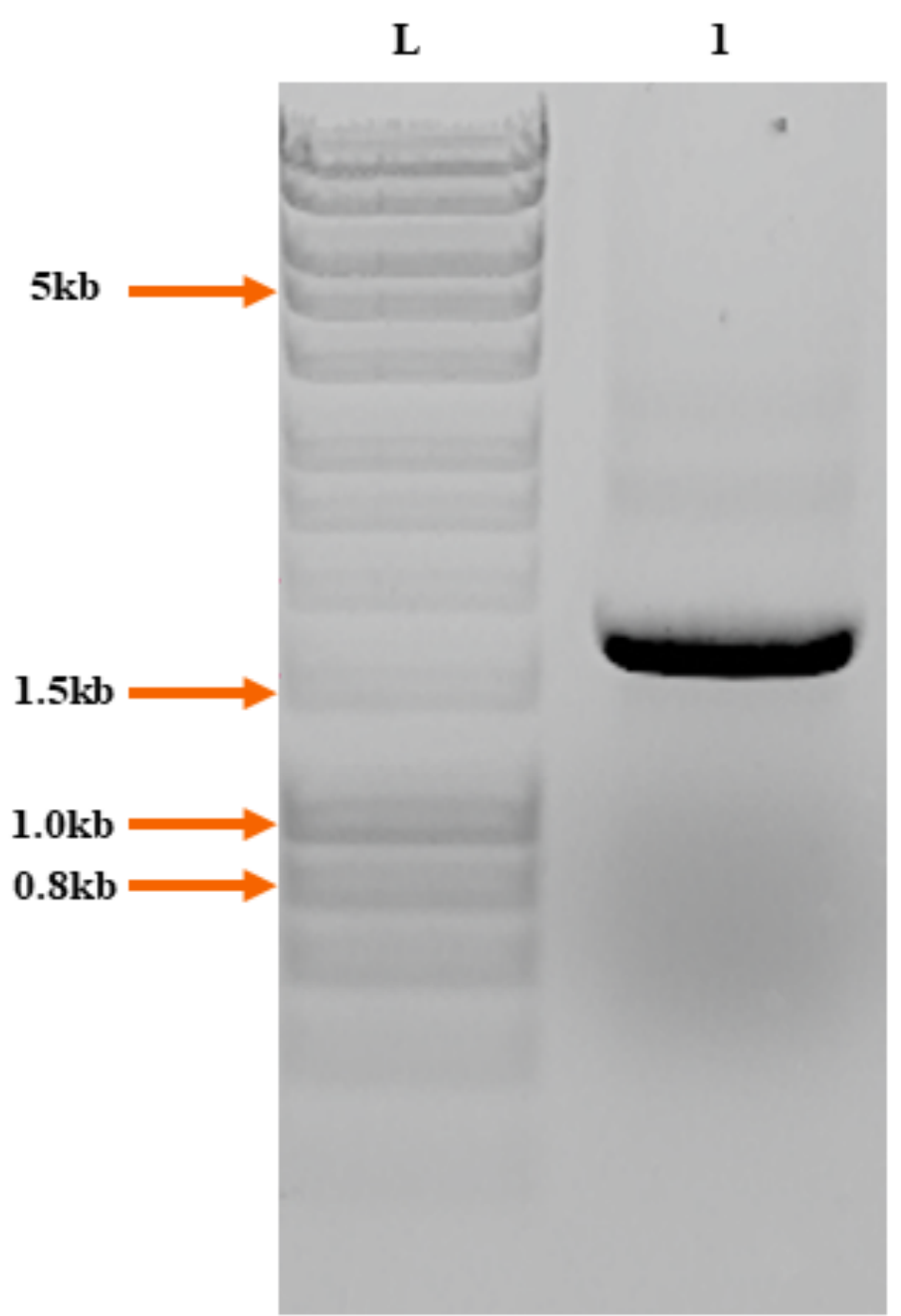

Figure 5.5 Purified PCR fusion product 1/4. Samples were run on a $1 \%$ agarose gel. The PCR fusion reaction yielded a product approximately $1.6 \mathrm{~kb}$ in size which corresponds to the expected size of the PCR fusion product as 1,658 bp (Lane 1). A strong band is seen at slightly above the $1.5 \mathrm{~kb}$ mark. The ladder (L) used is the Hyperladder $1 \mathrm{~kb}$ (BIOLINE). 
Now that we that we had our PCR fusion product, we could proceed to clone this product into our B. quintana suicide vector, $\mathrm{pEX} 18 \mathrm{Gm}$. We digested three samples of the vector with BamHI and one sample of the fusion product with BglII restriction enzyme overnight. The following morning, the digested $\mathrm{pEX} 18 \mathrm{Gm}$ vectors were treated with cIAP, to prevent re-closure of the vector. All of the digests were run on a DNA agarose gel for gel purification. The gel was viewed under a UV imager (Figure 5.6). The pEX18Gm plasmid is $5,831 \mathrm{bp}$ in size.

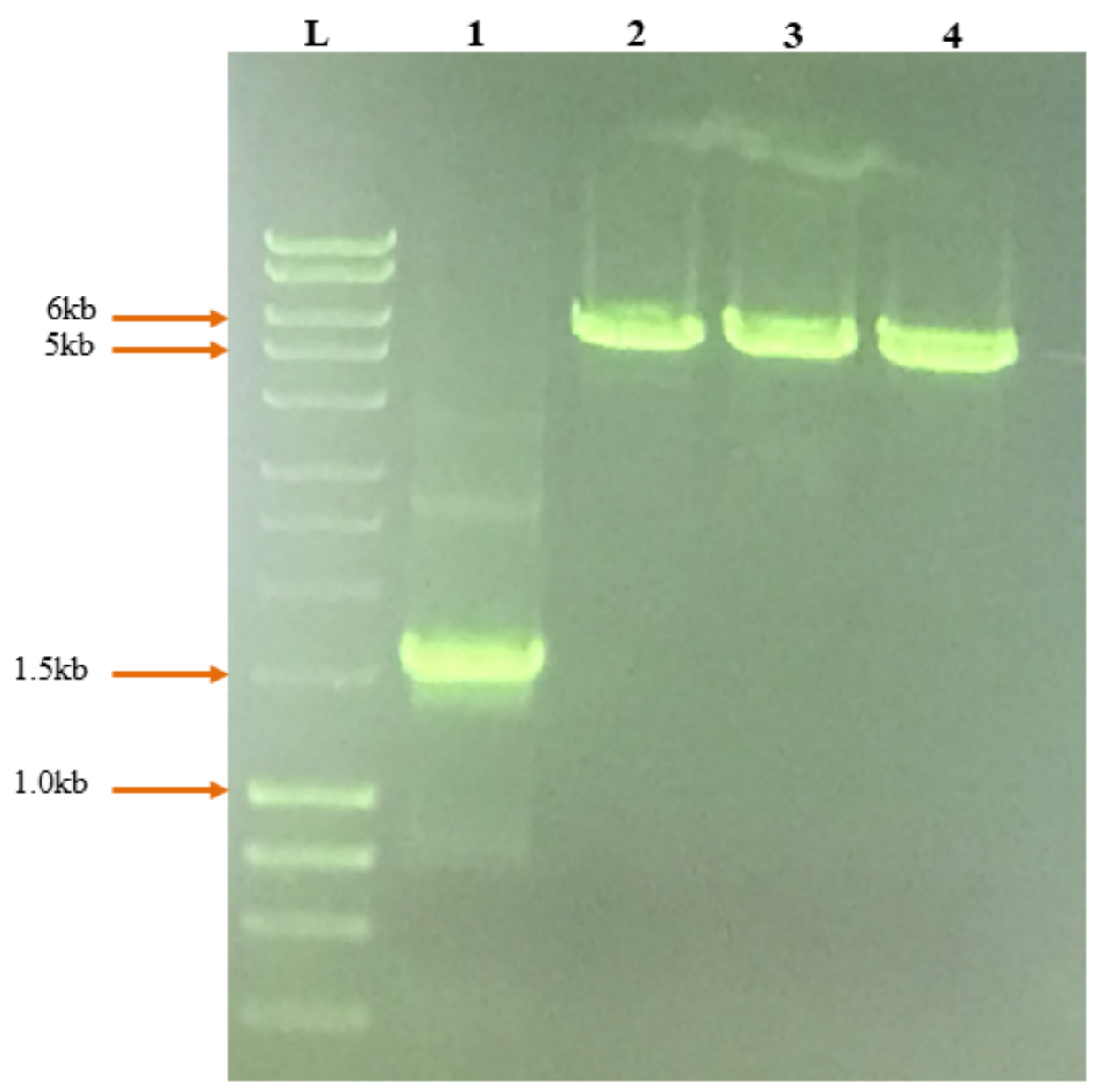

Figure 5.6 Digest of the PCR fusion product $1 / 4$ and pEX18Gm vector. After overnight digestion, the fusion product (Lane 1) and three samples of the pEX18Gm vector (Lanes 2, 3, 4) were run out on $1 \%$ agarose for gel purification 1 . The fusion product can be seen at approximately $1.6 \mathrm{~kb}$. The ladder (L) used is the Hyperladder $1 \mathrm{~kb}$ (BIOLINE).

After the overnight digestion, we saw strong bands for our fusion product and each pEX18Gm vector sample. The bands were carefully excised from the gel and purified. One of the digested pEX18Gm vectors was combined with the PCR fusion product $1 / 4$ in a ligation reaction. Additionally, we included two vector-only controls, one where ligase was added (no 
insert), and a second with vector only, but without ligase (no insert, no ligase). The inclusion of these two controls allowed us to check if the restriction enzymes digested the PCR fusion product to completion and to determine if there was religation and closing of the vector. The ligations were allowed to proceed overnight; the following morning, a small sample of each ligation was run out, alongside samples of the gel-purified but non-ligated bands, as shown in Figure 5.7 below.

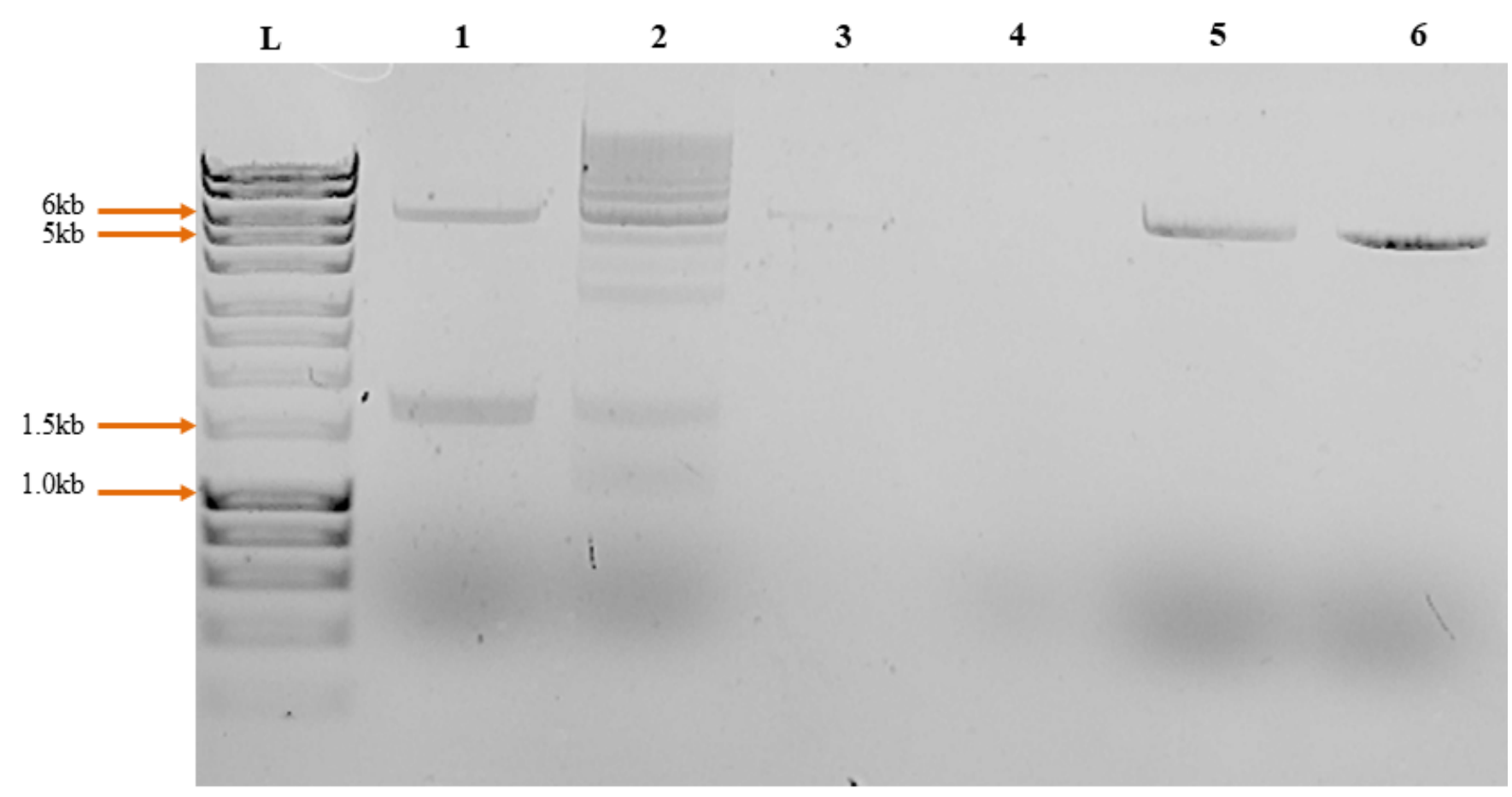

Figure 5.7 Gel analysis of the ligations of the PCR fusion product $1 / 4$ and the pEX18Gm vector samples. Samples were run on a 1\% agarose gel. Lane 1: combined digested PCR fusion product 1/4 insert and vector, pEX18Gm, prior to ligation. Lane 2: pEX18Gm + PCR fusion product 1/4 after ligation reaction; many higher molecular weight species are seen, indicating the ligase worked well. Lane 3: digested pEX18Gm (no insert), before overnight ligation. Lane 4: digested pEX18Gm vector (no insert), after overnight ligation. Both lanes 3 and 4 contain no products for reasons we are unable to explain. Lane 5: digested pEX18Gm (no insert, no ligase), before overnight "ligation". Lane 6: digested pEX18Gm (no insert, no ligase) after overnight "ligation". Lanes 5 and 6 contain vector only. The smear at the bottom of the gel wells is the UV shadow from the DNA loading dye. The ladder used (L) is the Hyperladder $1 \mathrm{~kb}$ (BIOLINE).

From the gel image in Figure 5.7 there is evidence of a successful ligation of the PCR fusion product $1 / 4$ into the $\mathrm{pEX} 18 \mathrm{Gm}$ vector, which is visible as the appearance of multiple 
high molecular weight species in Lane 2. While lanes 3 and 4 contain no visible product, we would have expected to see the digested vector band at $5.8 \mathrm{~kb}$. As water replaced the ligase enzyme in lanes 5 and 6 , we only see the fully digested pEX18Gm vector in these lanes, with no evidence of ligation or the generation of larger molecular weight products.

The ligation reactions, including that with the PCR fusion product $1 / 4$ and the pEX18Gm vector, as well as the two vector-only controls, were transformed into the $10 \mathrm{G} E$. cloni cloning strain, using standard techniques. Colonies were selected on gentamicin plates which had X-gal solution spread onto them, to allow for easy determination of which colonies carrying a plasmid with a cloned insert. These colonies would be seen as white, due to a disruption of the laca gene. All other colonies would appear as blue. Sample plates are shown in Figure 5.8 below. 

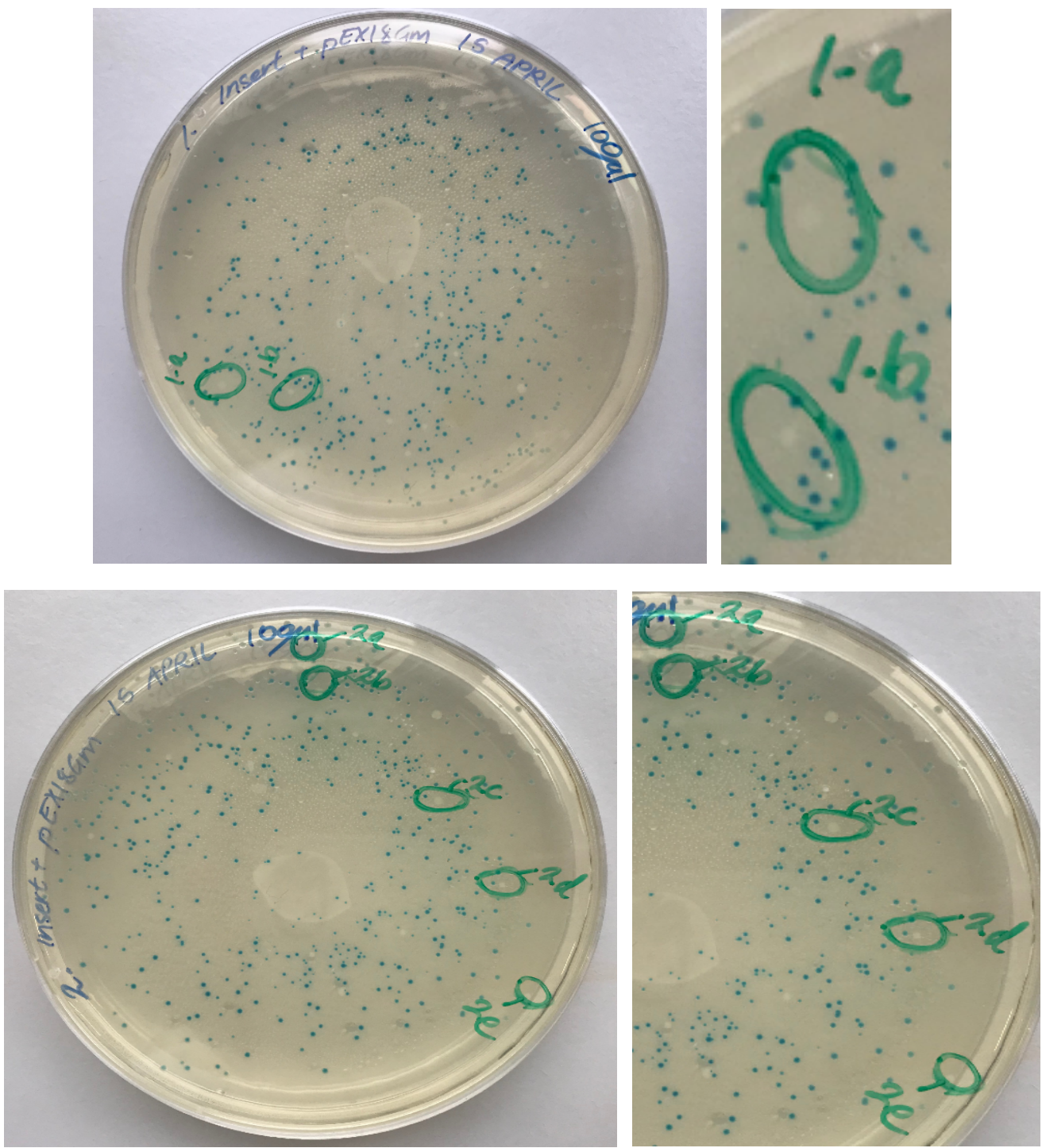

Figure 5.8 Electroporated $E$. coli (10G E. cloni) cells expressing the pEX18Gm_PCR fusion product 1/4. X-gal allowed the easy identification of white E. coli colonies which express the mutagenesis plasmid. White colonies from the vector and insert ligation transformations were selected for further manipulations. Green circles represent colonies selected for further screening.

The pEX18Gm vector-only controls yielded very few colonies, all of them blue, suggesting that vector digestion was thorough, and that the cIAP minimised plasmid religation. Selected white colonies from the transformations with both insert and vector were grown in liquid media overnight and subject to plasmid DNA purification the following day. Because cloning the BgIII-digested insert into a BamHI restriction site destroys both sites, the purified plasmid DNA from each colony was digested with restriction enzymes that flank BamHI in pEX18Gm. The plasmids were double digested with SmaI and SphI, at room temperature for one hour (optimal temperature for SmaI) and then at $37^{\circ} \mathrm{C}$ for another hour (optimal 
temperature for SphI). Each sample was then run on a DNA agarose gel to determine which colonies correctly expressed the pEX18Gm plasmid and the PCR fusion product $1 / 4$, as in seen in Figure 5.9 below.

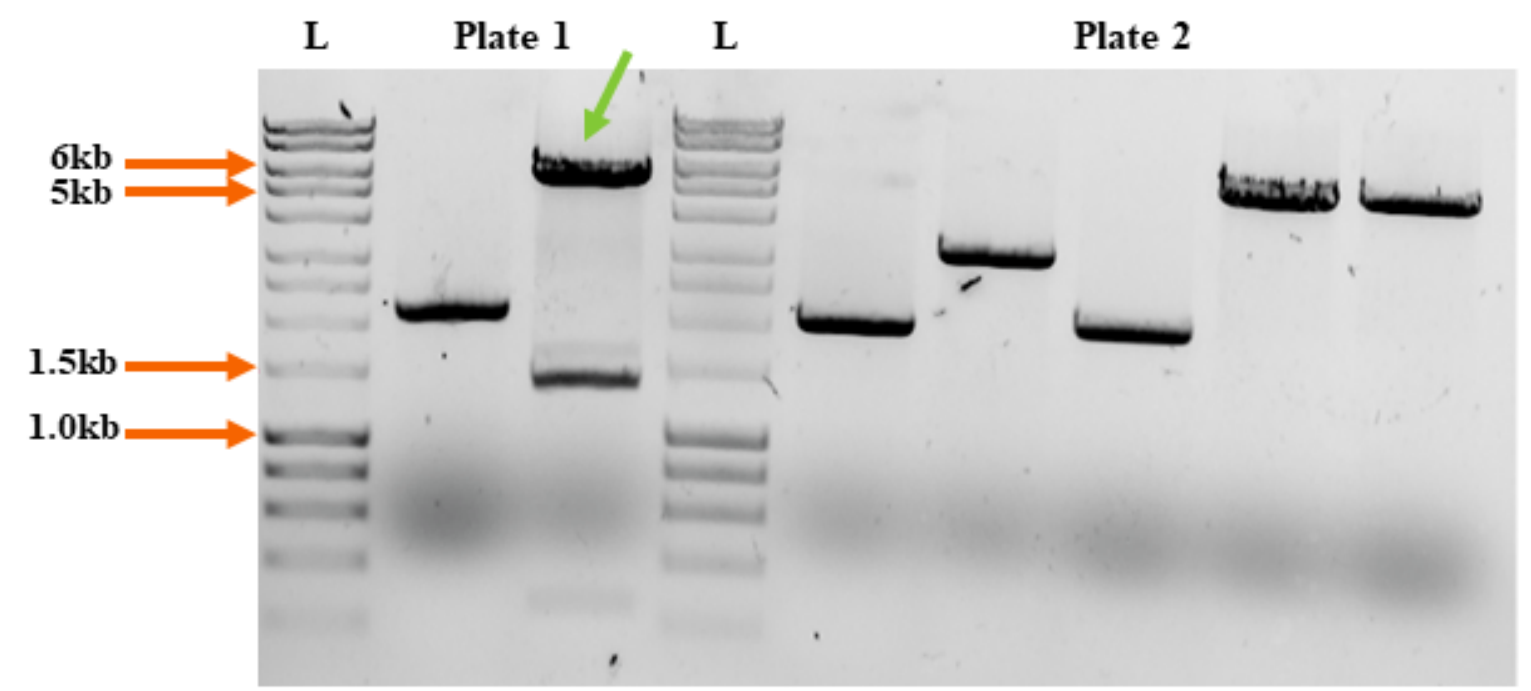

Figure 5.9 Agarose gel image of purified and digested plasmid from 10G E. cloni cells. The figure shows all the white colonies which were selected from the electroporated E. coli, grown, purified and digested. Only one of the colonies expressed the recombinant plasmid with the PCR fusion product 1/4, indicated by the green arrow.

From the gel analysis in Figure 5.9, it was evident that there was only one colony that correctly contained the BepA1_BepA2 insert in the pEX18Gm plasmid, indicated by the green arrow. The purified plasmid DNA from this colony was sequenced to ensure that it was correct (see Appendix Figure 7.7, which shows the complete sequence identity of the BepA1_BepA2 fusion product cloned into $\mathrm{pEX} 18 \mathrm{Gm})$. After sequence confirmation, the mutagenic plasmid was introduced into our E. coli conjugation strain, S17-1.

\subsubsection{Test conjugations}

Having found one colony correctly expressing the BepA1_BepA2 mutagenesis plasmid, we proceeded with a test conjugation prior to Bartonella conjugations. The rationale behind the test conjugation was to determine whether our mutagenesis plasmid could be mobilised into the recipient bacterium, thus confirming an intact oriT origin of transfer on pEX18Gm and intact RP4 conjugation machinery on the chromosome of S17-1.

For bacterial conjugations, our recipient strain was E. coli pACYC184, chosen for two reasons. Firstly, the pACYC184 plasmid contains a p15A origin of replication (ori), in contrast 
to the ColEI ori of pEX18Gm, and the plasmids are compatible in E. coli. Secondly, E. coli pACYC184 encodes chloramphenicol resistance while pEX18Gm encodes gentamicin resistance. As both our donor and recipient bacterium are both E. coli (E. coli S17-1 and E. coli pACYC184 respectively), we needed to distinguish between donor, recipient and the transconjugants. Successful conjugation between the E. coli S17-1 donor and the E. coli pACYC184 recipient would result in transconjugants with both chloramphenicol and gentamicin resistance. For each test conjugation, we also included a donor-only and a recipientonly negative control. Both of these negative controls were plated separately onto chloramphenicol-gentamicin plates. The results of one test conjugation are shown in Figure 5.10 below.

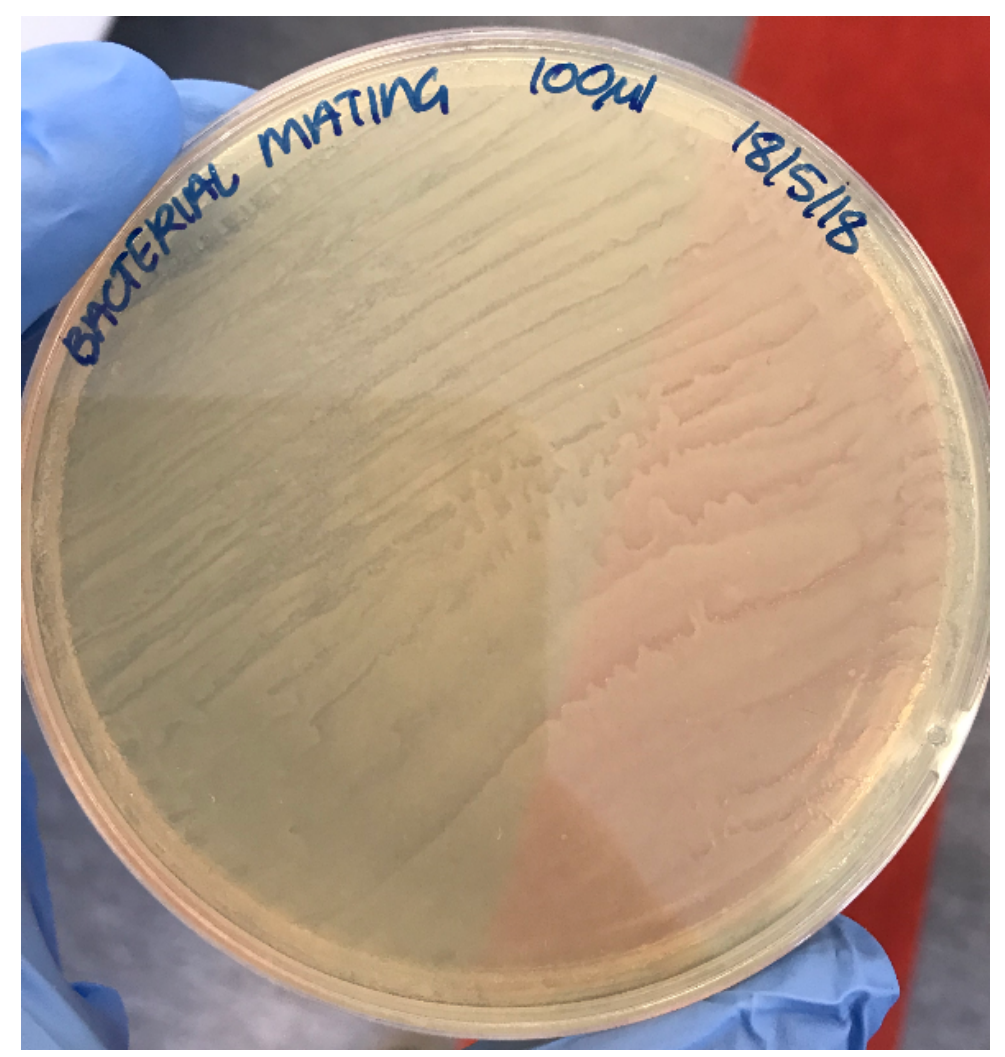

Figure 5.10: Plate containing successful bacterial mating between E. coli pACYC184 and S17-1 with pEX18Gm_BepA1. The conjugation was successful, with very high transformation efficiency, as seen by the lawn of growth of E. coli pACYC184 on a gentamicin and chloramphenicol plate.

The test conjugation yielded transconjugants on the chloramphenicol and gentamicin plates, too many to count, indicating the successful transfer of the BepA1_BepA2 mutagenesis 
plasmid into E. coli pACYC184. Neither of the negative controls yielded any colonies. The next step was to begin conjugations with $B$. quintana JK-31 as the recipient.

\subsubsection{B. quintana JK-31 conjugations}

Cultures of B. quintana JK-31 were revived from frozen stocks and passaged several times to generate healthy plates of recipient bacteria. The day before the conjugation, overnight liquid cultures were started of our conjugative strain, E. coli S17-1 expressing either the BepA1_BepA2 mutagenesis plasmid, or pSRKGm, our positive control as previously described (Methods, Table 2.1 Bacterial strains). For the B. quintana conjugations, negative controls were not included. However, we did include E. coli S17-1 carrying the pSRKGm vector, which was intended as a positive control for conjugation. pSRKGm is a broad-hostrange plasmid, capable of replicating in B. quintana; unlike the mutagenic plasmid, it does not need to be incorporated into the chromosome to confer gentamicin resistance. We included pSRKGm as we thought we might be more likely to obtain B. quintana transconjugant colonies than we would with just the BepA1_BepA2 mutagenesis plasmid.

Conjugations into B. quintana were started early in the morning. We made 1/10 dilutions of the overnight S17-1 liquid cultures, returning the cultures to the incubator until they reached an $\mathrm{OD}_{600}$ of $0.25-0.5$. For each conjugation, the cultures were allowed to reach an $\mathrm{OD}_{600}$ as close to 0.5 as possible and were not allowed to go over. Based the $\mathrm{OD}_{600}$, we calculated the exact volume of each culture required to for the conjugation. These volumes were dispensed into separate microcentrifuge tubes, spun at full speed, the supernatant removed and washed with M199S media thrice to remove residual antibiotics. After the last wash the pellets were not resuspended.

After preparing the E. coli S17-1 conjugative strains, the recipient B. quintana JK-31 strain was prepared. After allowing JK-31 to grow for $\sim 5$ days, the bacterial growth was harvested from 1-3 plates by scraping with a loop and resuspending in M199S media. A 1/100

dilution of the B. quintana suspension was used to measure the $\mathrm{OD}_{600}$. For each conjugation, we aimed for an $\mathrm{OD}_{600}$ of 30-40 for the JK-31 undiluted suspension, however we tried to achieve an $\mathrm{OD}_{600}$ of close to 40 each conjugation. JK-31 was washed one time with M199S, to minimise manipulation of the bacteria. The pellet was resuspended in sufficient M199S medium to have $50 \mu \mathrm{l}$ suspension per conjugation. The JK-31 suspension was then used to resuspend the E. coli S17-1 donor pellets. The combined donor and recipients were placed on the centre of non-selective chocolate plate, one per conjugation, to allow bacterial mating. 
Plates were allowed to air dry briefly before being placed and sealed in a candle jar, with a lit candle. The jar was incubated for 6 hours to allow for bacterial mating to occur. After the incubation, the entire bacterial growth on the conjugation plates was scraped and gently resuspended in M199S media to remove clumps. Dilutions of 1:5 of the bacterial suspensions were made in fresh M199S media, and $100 \mu \mathrm{L}$ plated onto chocolate plates, supplemented with gentamicin to select for plasmid maintenance or integration into the chromosome, and nalidixic acid and cefazolin antibiotics, both of which are counter-selections against S17-1. Plates were placed into a glass jar, a candle lit, and jar lid closed tightly. Plates were allowed to grow undisturbed for 2 weeks.

Over a period of 3 to 4 months, we made considerable efforts with making our BepA1_BepA2 deletion mutant with Bartonella conjugations. Each week we performed one conjugation, growing fresh B. quintana JK-31 and pouring fresh chocolate plates, performing this experiment to the best of our ability, handling the bacteria in the most sterile and hygienic conditions we were able to. Unfortunately, despite our numerous attempts (approximately 20 conjugations), no transconjugant colonies were obtained for either the BepA1 mutagenesis or the pSRKGm control plasmid.

During the early weeks of our Bartonella conjugation experiments, when we were consistently not seeing colonies on the bacterial mating plates, we tried to optimise the conjugation protocol. Small adjustments included using $2 x$ YT media instead of LB broth to grow the S17-1 strains, as $2 x$ YT media is more nutritionally dense than standard LB media and contains double the amount of yeast extract and supports a higher density of cells over a longer time. Accordingly, the use of $2 x \mathrm{YT}$ media resulted in a higher $\mathrm{OD}_{600}$ of E. coli $\mathrm{S} 17-1$ strains in the morning but did little to change the outcome of the conjugation as we still did not see colonies.

We next tried to extend the incubation time for the bacterial mating, increasing from 6 hours to 8 hours to allow the S17-1 ample time to mobilise the BepA1_BepA2 mutagenesis plasmid to JK-31. However, this also did not result in any colonies being obtained after the two-week incubation. We considered the option of allowing the bacterial mating to occur overnight, but as E. coli S17-1 quickly outcompetes JK-31 we did not implement this idea. One factor we kept consistent through our optimization of the conjugation protocol was ensuring that we had always freshly poured chocolate plates. B. quintana is a highly fastidious organism which grows best on chocolate plates that are no more than one week old. While we tested many optimizations, we never changed this factor. 
Thus, after many weeks of trialling and optimising our conjugation protocol, we were unsuccessful in generating our BepA1_BepA2 deletion mutant via bacterial conjugation. We eventually decided to stop with these conjugations as we were having little success and focused our efforts on other experiments. Time pressures meant that we were not able to explore the alternative methods to generate this BepA1_BepA2 deletion mutant. Conjugations have been often used in the field of Bartonella research with success by other researchers in the field, but our results suggested that our conjugation protocol was not working for B. quintana. Furthermore, the inclusion of the positive control with a self-replicating plasmid did not result in colonies further suggesting that the fault lies in the conjugation protocol. If the problem is a low rate of homologous recombination into the chromosome, we could potentially try increasing the length of the region of homology $\sim 800 \mathrm{bp}$ to $>1,000 \mathrm{bp}$. As there is no published data on the recommended length of how much homologous sequence is required for recombination is Bartonella, we may need to trial different lengths to see what may result in successful recombination.

Overall, our current conjugation protocol did not result in successful bacterial conjugation. While we based our conjugation protocol on published and communicated protocols, for some yet undetermined reason/s, this protocol did not yield transconjugants. Being unable to generate the BepA1_BepA2 deletion mutant was disappointing but from our results we have learnt that bacterial conjugation is perhaps not the method for generating our BepA1_BepA2 deletion mutant. However, we can still pursue other experimental procedures with which we may have more success. 


\subsection{Discussion}

We were able to successfully create our BepA1_BepA2 mutagenesis plasmid and introduce it into our conjugative strain, E. coli S17-1. The plasmid was also successfully and efficiently mobilised into an E. coli recipient strain. However, we were unable to generate the BepA1_BepA2 deletion mutant and time constraints made further conjugation experiments with $B$. quintana infeasible. While we did not have the BepA1_BepA2 deletion mutant to proceed with studying host-pathogen interactions, we knew that our unsuccessful efforts to generate this mutant via bacterial conjugation proved that a different approach is required, especially after we optimised the conjugation protocol as much as we could in the time frame that we had.

There are many alternative approaches to introduce foreign DNA into bacteria that could be trialled in the future, including electroporation, triparental conjugation and calcium chloride heat shock transformation, although only bacterial conjugation and electroporations have been reported for Bartonella $[118,153]$. Genetic transformations using calcium chloride have been used for genetically intractable organisms, such as Chlamydia trachomatis, and could be explored with Bartonella [154]. Had we more time, we would have proceeded to test both increasing the length of the homologous regions and testing a longer incubation time for bacterial mating, and if these didn't work, then we could trial electroporation, triparental conjugation, or other protocols.

Electroporation is a well-established method and has been used to successfully transform multiple Bartonella species [63, 153, 155]. It uses high voltage electric shocks to introduce DNA into cells and involves suspending host cells and plasmids, e.g. B. quintana and the pEX18Gm_BepA1 mutagenesis plasmid, in a conductive solution, usually a glycerol solution, in an electroporation cuvette [156]. An electrical current is applied at an optimized voltage lasting just a few microseconds, which disrupts the phospholipid bilayer of the cell membrane, resulting in temporary pores. This allows macromolecules such as DNA, to be driven across the membrane by the increased electric potential of the membrane [157]. Electroporation is advantageous because it can be used on a variety of cell types and it is easy to transform a large number of cells at a time. However, because of the high voltage pulse, this process also kills a large proportion of the cells. Therefore, it is recommended to use a larger starting quantity of cells and to immediately rescue them by the addition media such as SOC Medium, plain LB broth or $2 x$ YT media. Because B. quintana does not grow well in liquid, recovery overnight on plates could also be trialled. 
Triparental conjugation is another method to test. Triparental conjugations are an effective method for moving a nonconjugative but mobilisable plasmid, such as the pEX18Gm_BepA1_BepA2 mutagenesis plasmid. This method utilizes both helper and donor strains of $E$. coli to move the plasmid of interest into the recipient bacterium. The helper E. coli strain carries a plasmid that expresses conjugative machinery, which enables production of a mating bridge and transfer of the plasmid into the recipient strain [158]. This involves conjugation involves between the helper and donor E. coli strains so that the donor can express the helper plasmid, and then a conjugation between the donor and the recipient bacteria to transfer the engineered plasmid into the recipient bacterium. This process is summarised in Figure 5.11 below.

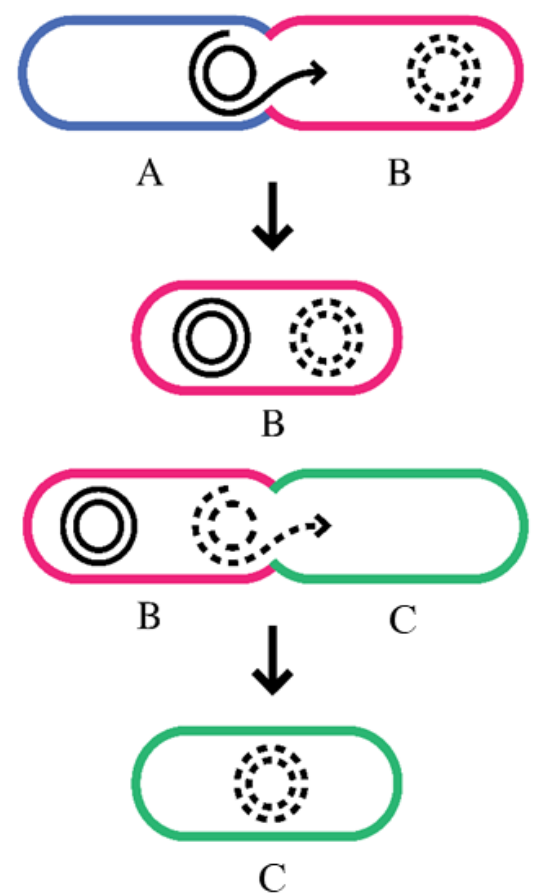

Figure 5.11 Triparental conjugation strategy. Triparental conjugation utilises helper and donor strains of $E$. coli to transfer the engineered plasmid into a recipient bacterium. The $E$. coli helper strain contains a plasmid with transfer and mobilisation functions, which aid in mobilising non-selftransmissible plasmids. The $E$. coli donor strain carries the engineered plasmid which is mobilisable but not self-transmissible. First, a conjugation between the E. coli helper strain A, and the E. coli donor strain $\mathbf{B}$, results in the mobilisation of the helper plasmid (solid circles) to the donor E. coli. The donor $E$. coli now expresses both the helper plasmid and the engineered plasmid (dashed circles). A second conjugation between the donor $E$. coli strain and the recipient bacterium $\mathbf{C}$, results in the transfer of the engineered plasmid to the recipient bacterium, aided by the transfer and mobilisation functions of the helper plasmid. The recipient bacterium, $\mathbf{C}$, now carries the engineered plasmid, while the helper plasmid is lost. Figure taken and adapted with permission from Wise et al. 2006 [158]. 
This strategy has been utilized by Bartonella researchers with success [59, 159, 160]. The helper plasmid that is used here is E. coli expressing the pRK2013 plasmid, which has been used in many triparental conjugations (see, for a selection: $[161,162]$ ). The wealth of published data about triparental conjugations makes this a tempting future experiment to test with B. quintana JK-31 to help generate our BepA1_BepA2 deletion mutant, and further our study around host-pathogen interactions.

Recently, our lab has successfully established a protocol for electroporations with $B$. quintana. This is an exciting development and now allows us to pursue creating our BepA1_BepA2 B. quintana deletion mutant. There are many available opportunities to test experimental procedures to generate the BepA1_BepA2 mutagenesis plasmid. Further work would allow us to provide insight into the fascinating but understudied B. quintana effector proteins, BepA1 and BepA2, and the explore their full impact on host-pathogen interactions, knowledge that is urgently needed to shed light on the complicated intracellular survival and persistence mechanisms the of this re-emerging stealth pathogen. 


\section{Chapter 6 Research motivations, key findings and future directions}

\subsection{Research motivations}

Bartonella are virtually ubiquitous among mammals, especially when arthropod ectoparasites are prevalent, despite very limited public awareness [11]. These zoonotic opportunistic pathogens pose a significant risk to public health, placing vulnerable individuals at risk of an asymptomatic bacteraemia which can develop to more serious symptoms and disease. Furthermore, the host survival and persistence mechanisms of Bartonella are not very well understood, leaving significant gaps in our understanding of their biology.

The recent clusters of $B$. quintana cases in vulnerable individuals once again place the pathogen on the radar of epidemiologists and researchers worldwide, prompting an urgent increase in biomedical research.

Bartonella, like many bacterial pathogens, harbour effector proteins deployed upon host infection. Many Bartonella effector proteins contain one at least one FIC or BID domain. FIC domains have been recently shown to confer a pathogenic function in various bacterial pathogens e.g. VopS from $V$. parahaemolyticus, Ank from L. pneumophilia, and IbpA from $H$. somni, where AMPylation targets host GTPase proteins that are involved in maintaining the function of the actin cytoskeleton. A longstanding question has been whether any Bartonella species harbour a FIC domain protein involved in pathogenesis.

My motivation for this research arose from the significant gaps in our knowledge and understanding of the survival and persistence of B. quintana within the reservoir human host. While there have been modern efforts to better understand these processes, B. quintana remains a neglected pathogen, even though it is one of the three clinically relevant Bartonella species to cause disease in humans. Some of the larger questions surrounding Bartonella infection remain unanswered, including but not limited to: what immune pathways is this bacterium modulating to enable its long-term intracellular survival and persistence, and what bacterial effectors are being deployed? 


\subsection{Key findings}

Preliminary research in our lab suggested an immunomodulatory function for $B$. quintana BepA1, however, the experiments were preliminary and required further experimental validation. Thus, addressing these gaps in our knowledge of Bartonella biology formed the basis of the BepA1 project.

In this study we used an in vitro human cell model to characterise the innate immune responses of HeLa cells when transfected with the B. quintana WT BepA1 protein and a catalytically dead BepA1 FIC domain mutant. Our research provides necessary insight into the innate immune responses potentially targeted by B. quintana via its effector, BepA1, to enable its intracellular survival and persistence within the reservoir human host. Through qPCR experiments we aimed to validate these observations, though future work is required to elucidate the downstream targets of BepA1 and elaborate on the full implications of BepA1 as an effector deployed during reservoir host infection.

We hypothesised that $B$. quintana's BepA1 protein would depress the secretion of proinflammatory cytokines and chemokines in HeLa cells, compared to the BepA1 FIC domain mutant transfected HeLa cells. Our results suggest that BepA1 is able to modulate the expression of proinflammatory cytokines and chemokines, particularly those that function to recruit immune cells to the site of infection or promote a proinflammatory state e.g. IL-6 and IL-8.

With these preliminary results, we pursued quantitative PCR experiments to further validate the results of the Proteome Profiler Human Cytokine Array and to provide information on whether the expression of select cytokines, IL-6 and IL-8 and an SRF-regulated gene, SM22, is altered in the presence of B. quintana BepA1, compared to a FIC domain mutant BepA1 protein.

qPCR helped to validate the observations that we had seen, providing additional support to the observation that BepA1 is suppressing the expression of IL-6 and IL-8, while this ability is lost in the BepA1 FIC domain mutant protein.

Together, the Human Cytokine Array assay and the qPCR experiments showed that an intact FIC domain is required for the phenotypes we observed.

Additionally, we demonstrated that HeLa cells produce an mRNA transcript of the cardiac protein, Myozap. Additional protein interaction studies can be carried out to investigate the role of Myozap in the interactions of BepA1 and the host. 


\section{BepA1 interaction with transcription factors}

Based on the results of the Proteome Profiler Assay, the nine cytokines and chemokines whose expression differs between BepA1 WT and FIC domain mutant are transcriptionally regulated through the transcription factors, NF-KB and/or AP-1, both of which may be activated by TNF- $\alpha$. This was fascinating because if BepA1 is targeting NF-KB or AP-1, then it will be the first bacterial FIC domain effector protein that targets these transcription factors. Our work suggests that NF-KB activation is altered by BepA1 via mechanisms that remain unclear. Thus, the mechanism of NF-kB alteration would be a promising candidate for future studies.

\section{Wild-type BepA1 protein interaction with NFkB}

Before we can confirm that the WT BepA1 protein suppresses the expression of IL-6 and IL-8 we need to understand at which point within the NF- $\kappa B$ signalling pathway is expression being inhibited. Because NF- $\mathrm{B}$ is a very well-studied family of transcription factors we can conduct reporter assays to test whether the WT BepA1 protein affects expression of NF-кB promoters. Thus, we first need to answer, does WT BepA1 block expression from all NF-KB promoters?

To test this, a luciferase reporter assay can be utilised. Luciferase is an enzyme which is produced by many organisms in nature for bioluminescence e.g. fireflies. For in vitro purposes, this bioluminescence is used as an indicator of whether expression from a promoter has been affected by a variable, such as the introduction of a bacterial effector. Luciferase is an enzyme which catalyses the conversion of its substrate luciferin to oxyluciferin and this reaction results in the release of light, which can be detected using a luminometer to precisely quantify the amount of light produced [163]. Because the target gene is cloned directly upstream of the luciferase reporter gene, the amount of light produced can be directly quantified to the transcription of the target gene. Many luciferase assay kits are commercially available.

To perform this assay, a plasmid with a luciferase reporter gene immediately downstream of NF- $\kappa \mathrm{B}$ response elements, can be purchased. Activation of NF- $\kappa \mathrm{B}$ will produce oxyluciferin, and a luminometer could detect and quantify light. This construct could be used together with plasmids expressing BepA1, wild type or FIC mutant. Using a transfection culture model, a double transfection would be performed, including controls.

If WT BepA1 blocks the expression of NF- $\mathrm{BB}$ from all promoters, then the amount of

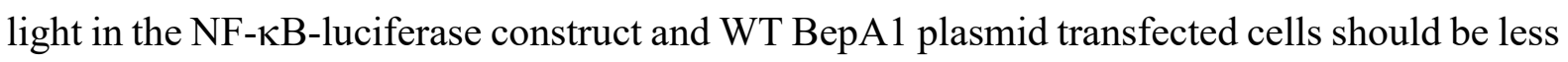


compared to the light emitted from TNF- $\alpha$-stimulated cells with the NF- $\kappa \mathrm{B}$-luciferase

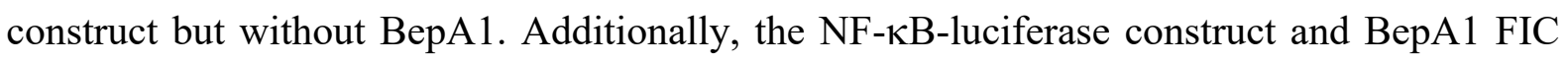
domain mutant plasmid transfected cells should not show any inhibition of NF-kB expression and the bioluminescence reading should be similar to just NF- $\kappa \mathrm{B}$-luciferase construct transfected cells.

This assay could also be adapted to an infection model, with light production from cells transfected with the reporter plasmid compared for cell infected with B. quintana wild type vs the BepA1 mutant. These experiments would show if the WT BepA1 protein is interfering in $\mathrm{NF}-\kappa \mathrm{B}$ activation of promoters. If the results indicate that the WT BepA1 protein is blocking $\mathrm{NF}-\kappa \mathrm{B}-$ mediated transcription, we would next ask if BepA1 blocks the translocation of NF- $\kappa \mathrm{B}$ into the nucleus.

\section{NF-kB translocation into the nucleus}

NF-KB is a family of 5 structurally related inducible transcription factors that mediate the transcription of target genes by binding to their specific DNA element, the $\mathrm{kB}$ enhancer, as various hetero-or homodimers $[164,165]$. These NF-kB proteins, including $\mathrm{p} 65$, are normally sequestered in the cell cytoplasm bound by a family of inhibitory proteins - the Iк $\beta$ family and their related proteins. Translocation of $\mathrm{p} 65$ into the nucleus can be monitored by fluorescence microscopy or subcellular fractionation and immunoblot.

In order for NF-кB to translocate into the nucleus, the Ік $\beta$ inhibitor must be degraded. Therefore, we can also ask if BepA1 WT blocks the degradation of the Iк $\beta$ inhibitor, thereby blocking the translocation of NF-KB into the nucleus. Iк $\beta$ degradation can be monitored by immunoblot following TNF- $\alpha$ stimulation.

If $\mathrm{NF}-\kappa \mathrm{B}$ nuclear translocation is occurring as usual, we can also test if the transcription factors bind to the $\mathrm{\kappa B}$ response elements in the nucleus, by chIP-qPCR, immunoprecipitation and immunoblotting experiments. A chIP assay (chromatin immunoprecipitation) a method of testing the interaction of a transcription factor with its target genes in the native context of the cell - bound to chromatin in the nucleus [166]. chIP is a powerful tool and can be used in combination with molecular biology techniques such as PCR and real-time PCR, gene cloning and DNA microarrays to determine if the transcription factor is bound to its target native chromatin [166]. Many studies have utilised chIP-qPCR to analyse transcription factor activity [167-170]. 
For using chIP-qPCR to test whether NF-kB interacts with its promoter elements in the presence of the BepA1 WT protein, we would transfect HeLa cells with the BepA1 WT protein and the FIC domain mutant as has been described here, and then isolate the NF- $\kappa \mathrm{B}$ transcription factor and ask if it is bound to its target DNA sequence, using qPCR analysis.

If p65 is in the nucleus but not bound to the target DNA sequence, this suggests the WT BepA1 protein interferes with the interaction of nuclear NF- $\kappa \mathrm{B}$ with its promotes, as other transcription factors have been shown to do [171]. Further experiments could be conducted to isolate at which precise point BepA1 protein may be affecting NF- $\kappa \mathrm{B}$.

\section{B. quintana infection models}

Infection models are an incredibly useful tool to study bacterial pathogenesis, particularly when we can replicate the course of infection in an animal host or tissue culture cell line. With $B$. quintana, the only animal model is the rhesus macaque, which makes infection models technically challenging and expensive. Therefore, to study the impact of $B$. quintana bacterial effectors, including BepA1 and BepA2, a tissue culture infection model can be used. These experiments would involve culturing an appropriate cell line, e.g., HeLa-229, or primary vascular endothelial cells, and infecting these cells with a bacterial load of the WT B. quintana JK-31 strain, the BepA1_BepA2 deletion mutant, and complemented versions of the mutant [172]. Immune responses can be assessed both with and without TNF- $\alpha$ stimulation.

Naturally, the introduction of bacteria to an in vitro cell culture would elicit numerous innate immune responses, which could be measured and compared via different methods. To measure these innate immune responses either or both cell supernatants and cell lysates could be used to probe for many or select cytokines and chemokines. The use of the Human Cytokine Array Assay followed by qPCR gene expression analysis is an attractive approach, as these methods have been used in the current study to characterise innate immune responses.

This infection model would allow us to further validate that BepA1 is inhibiting cytokine and chemokine expression in vitro. 
Elucidating the host-pathogens interactions between $B$. quintana and its reservoir host is fundamental to better understand the mechanisms by which B. quintana can survive and persist in the host for months or even years. The increasing number of recorded B. quintana cases should prompt an increase in biomedical research surrounding this stealth pathogen. Our study aimed to broadly characterise the innate immune responses of human cells when transfected with the $B$. quintana effector protein BepAl and compare responses to a catalytically dead BepA1 FIC domain mutant.

We present novel findings: B. quintana's BepA1 is the first characterised FIC domain protein to encode an immunosuppressive function whereby BepA1 inhibited the expression of cytokines and chemokines in transfected HeLa cells following stimulation by TNF- $\alpha$. The inclusion of a catalytically dead BepA1 FIC domain showed that the FIC domain must be functional for the observed immunosuppressive effect to be operative. These results remain preliminary, and additional experiments are needed to further validate and explore these findings. Our research provides exciting and much needed insight into how B. quintana manipulates host innate immune responses in its favour during early infection. 
1. Ettema., T.J.G. et al., The alpha-proteobacteria: the Darwin finches of the bacterial world. Biology letters, 2009. 5(3): p. 429-432.

2. Álvarez-Fernández., A., et al., Bartonella infections in cats and dogs including zoonotic aspects. Parasites \& vectors, 2018. 11(1): p. 624.

3. Jones., K.E., et al., Global trends in emerging infectious diseases. Nature, 2008. 451(7181): p. 990-993.

4. Breitschwerdt., E.B., Bartonellosis: One Health Perspectives for an Emerging Infectious Disease. ILAR Journal, 2014. 55(1): p. 46-58.

5. Fournier, P.-E., et al., Bartonella australis sp. nov. from kangaroos, Australia. Emerging infectious diseases, 2007. 13(12): p. 1961-1962.

6. Carrasco., S.E., et al., Novel Bartonella infection in northern and southern sea otters (Enhydra lutris kenyoni and Enhydra lutris nereis). Veterinary Microbiology, 2014. 170(3): p. 325-334.

7. Cheslock., M.A. et al., Human Bartonellosis: An Underappreciated Public Health Problem? Tropical Medicine and Infectious Disease, 2019. 4(2): p. 69.

8. $\quad$ Eicher., S.C. et al., Bartonella entry mechanisms into mammalian host cells. Cellular Microbiology, 2012. 14(8): p. 1166-1173.

9. Billeter., S.A., et al., Vector transmission of Bartonella species with emphasis on the potential for tick transmission. Medical and Veterinary Entomology, 2008. 22(1): p. 115.

10. Guy., L., et al., A gene transfer agent and a dynamic repertoire of secretion systems hold the keys to the explosive radiation of the emerging pathogen Bartonella. PLOS Genetics, 2013. 9(3): p. e1003393-e1003393.

11. Harms., A. et al., Intruders below the radar: molecular pathogenesis of Bartonella spp. Clinical microbiology reviews, 2012. 25(1): p. 42-78.

12. Minnick., M.F., et al., Oroya fever and verruga peruana: bartonelloses unique to South America. PLOS Neglected Tropical Diseases, 2014. 8(7): p. e2919-e2919.

13. Dehio., C., et al., Emerging bartonellosis. Microbiology Today, 2003. 30: p. 168-169.

14. Chamberlin., J., et al., Epidemiology of Endemic Bartonella bacilliformis: A Prospective Cohort Study in a Peruvian Mountain Valley Community. The Journal of Infectious Diseases, 2002. 186(7): p. 983-990.

15. Karem., K.L., et al., Regnery, Bartonella henselae, B. quintana, and B. bacilliformis: historical pathogens of emerging significance. Microbes and Infection, 2000. 2(10): p. 1193-1205.

16. Mazur-Melewska., K., et al., Cat-scratch disease: a wide spectrum of clinical pictures. Postepy dermatologii i alergologii, 2015. 32(3): p. 216-220.

17. Opavsky., M.A., Cat scratch disease: The story continues. The Canadian journal of infectious diseases (Journal canadien des maladies infectieuses), 1997. 8(1): p. 43-49.

18. Brenner, D.J., et al., Proposals To Unify the Genera Bartonella and Rochalimaea, with Descriptions of Bartonella quintana comb. nov., Bartonella vinsonii comb. nov., Bartonella henselae comb. nov., and Bartonella elizabethae comb. nov., and To Remove the Family Bartonellaceae from the Order Rickettsiales. International Journal of Systematic and Evolutionary Microbiology, 1993. 43(4): p. 777-786.

19. Zangwill K. M. Cat scratch disease and other Bartonella infections. Advances in experimental medicine and biology, 2013. 764, p. 159-166.

20. Foucault, C., et al., Bartonella quintana Bacteremia among Homeless People. Clinical Infectious Diseases, 2002. 35(6): p. 684-689. 
21. Drancourt., M., et al., Bartonella quintana in a 4000-Year-Old Human Tooth. The Journal of Infectious Diseases, 2005. 191(4): p. 607-611.

22. Nguyen-Hieu., T., et al., Evidence of a louse-borne outbreak involving typhus in Douai, 1710-1712 during the war of Spanish succession. PLOS one, 2010. 5(10): p. e15405e15405.

23. Raoult., D., et al., Evidence for Louse-Transmitted Diseases in Soldiers of Napoleon's Grand Army in Vilnius. The Journal of Infectious Diseases, 2006. 193(1): p. 112-120.

24. Anstead., G.M., The centenary of the discovery of trench fever, an emerging infectious disease of World War 1. The Lancet Infectious Diseases, 2016. 16(8): p. e164-e172.

25. Foucault., C., et al., Bartonella quintana characteristics and clinical management. Emerging infectious diseases, 2006. 12(2): p. 217-223.

26. Ruiz., J., Bartonella quintana, past, present, and future of the scourge of World War I. APMIS, 2018. 126(11): p. 831-837.

27. McNee., J.W. et al., “TRENCH FEVER”. British Medical Journal, 1916. 1(2876): p. 225.

28. Bruce., D., Trench Fever: Final Report of the War Office Trench Fever Investigation Committee. The Journal of hygiene, 1921. 20(3): p. 258-288.

29. Vinson., J.W., et al., Trench fever. 3. Induction of clinical disease in volunteers inoculated with Rickettsia quintana propagated on blood agar. American Journal of Tropical Medicine and Hygiene, 1969. 18(5): p. 713-722.

30. Ohl., M.E. et al., Bartonella quintana and Urban Trench Fever. Clinical Infectious Diseases, 2000. 31(1): p. 131-135.

31. Jackson., L.A. et al., Emergence of Bartonella quintana infection among homeless persons. Emerging infectious diseases, 1996. 2(2): p. 141-144.

32. Jackson., L.A., et al., Seroprevalence to Bartonella quintana among Patients at a Community Clinic in Downtown Seattle. The Journal of Infectious Diseases, 1996. 173(4): p. 1023-1026.

33. Brouqui., P., et al., Chronic Bartonella quintana Bacteremia in Homeless Patients. New England Journal of Medicine, 1999. 340(3): p. 184-189.

34. Drancourt., M., et al., Bartonella (Rochalimaea) quintana Endocarditis in Three Homeless Men. New England Journal of Medicine, 1995. 332(7): p. 419-423.

35. Davila., V. et al., Rare infectious diseases are rising at an 'alarming' rate in Seattle's homeless population, concerning health officials. 2018.

36. Desk., N. Seattle area: Bartonella, Group A strep infections on the rise among the homeless. 2018.

37. Hawryluk., M., An Ickier Outbreak: Trench Fever Spread by Lice Is Found in Denver. 2020.

38. Koehler., J.E., Bartonella-associated infections in HIV-infected patients. AIDS Clinical Care, 1995. 7(12): p. 97-102.

39. Stoler., M.H., et al., An Atypical Subcutaneous Infection Associated with Acquired Immune Deficiency Syndrome. American Journal of Clinical Pathology, 1983. 80(5): p. 714-718.

40. Relman., D.A., et al., The agent of bacillary angiomatosis. An approach to the identification of uncultured pathogens. The New English Journal Medicine, 1990. 323(23): p. 1573-1580.

41. Chu., V.H., Endocarditis. JAMA, 2018. 320(1): p. 102-102.

42. Lepidi., H., et al., Quantitative Analysis of Valvular Lesions During Bartonella Endocarditis. American Journal of Clinical Pathology, 2000. 114(6): p. 880-889. 
43. Rolain., J.M., et al., Recommendations for treatment of human infections caused by Bartonella species. Antimicrobial agents and Chemotherapy, 2004. 48(6): p. 19211933.

44. Angelakis., E. et al., Pathogenicity and treatment of Bartonella infections. International Journal of Antimicrobial Agents, 2014. 44(1): p. 16-25.

45. Agan., B.K. et al., Laboratory diagnosis of Bartonella infections. Clinics in Laboratory Medicine, 2002. 22(4): p. 937-962.

46. Pachirat., O, et al., Echocardiographic Features in Bartonella Endocarditis: A Case Series. Cardiology research, 2018.9(2): p. 116-119.

47. Siamer., S. et al., New insights into the role of Bartonella effector proteins in pathogenesis. Current Opinion in Microbiology, 2015. 23: p. 80-85.

48. Chiaraviglio., L., et al., An immunocompromised murine model of chronic Bartonella infection. The American Journal of Pathology, 2010. 176(6): p. 2753-2763.

49. Brouqui., P. et al., Bartonella quintana invades and multiplies within endothelial cells in vitro and in vivo and forms intracellular blebs. Research in Microbiology, 1996. 147(9): p. 719-731.

50. Kempf., V.A., et al., Interaction of Bartonella henselae with endothelial cells results in rapid bacterial rRNA synthesis and replication. Cellular Microbiology, 2000. 2(5): p. 431-441.

51. Verma., A., et al., Infection of human endothelial cells with Bartonella bacilliformis is dependent on Rho and results in activation of Rho. Infection and immunity, 2000. 68(10): p. 5960-5969.

52. Zhang., P., et al., A family of variably expressed outer-membrane proteins (Vomp) mediates adhesion and autoaggregation in Bartonella quintana. Proceedings of the National Academy of Sciences of the United States of America, 2004. 101(37): p. 13630-13635.

53. Hong., J., et al., Lymphatic Circulation Disseminates Bartonella Infection Into Bloodstream. The Journal of Infectious Diseases, 2016. 215(2): p. 303-311.

54. Dehio., C., Bartonella interactions with endothelial cells and erythrocytes. Trends in Microbiology, 2001. 9(6): p. 279-285.

55. Clayton., K., et al., Langerhans Cells-Programmed by the Epidermis. Frontiers in Immunology, 2017. 8(1676).

56. Malgorzata-Miller., G., et al., Bartonella quintana lipopolysaccharide (LPS): structure and characteristics of a potent TLR4 antagonist for in-vitro and in-vivo applications. Scientific Reports, 2016. 6(1): p. 34221.

57. Whitfield., C., et al., Biosynthesis and export of bacterial lipopolysaccharides. Annual Review of Biochemistry, 2014. 83: p. 99-128.

58. Popa., C., et al., Bartonella quintana lipopolysaccharide is a natural antagonist of Tolllike receptor 4. Infection and immunity, 2007. 75(10): p. 4831-4837.

59. MacKichan., J.K., et al., A SacB mutagenesis strategy reveals that the Bartonella quintana variably expressed outer membrane proteins are required for bloodstream infection of the host. Infection and Immunity, 2008. 76(2): p. 788-795.

60. Thibau., A., et al., (2020). Immunogenicity of trimeric autotransporter adhesins and their potential as vaccine targets. Medical Microbiology and Immunology, 209(3), 243-263.

61. Schröder., G., et al., Conjugative DNA transfer into human cells by the VirB/VirD4 type IV secretion system of the bacterial pathogen Bartonella henselae. Proceedings of the National Academy of Sciences, 2011. 108(35): p. 14643-14648.

62. Wallden., K., et al., Type IV secretion systems: versatility and diversity in function. Cellular Microbiology, 2010. 12(9): p. 1203-1212. 
63. Vayssier-Taussat., M., et al., The Trw type IV secretion system of Bartonella mediates host-specific adhesion to erythrocytes. PLOS Pathogens, 2010. 6(6): p. e1000946e1000946.

64. Christie., P.J., et al., Mechanism and structure of the bacterial type IV secretion systems. Biochimica et biophysica acta, 2014. 1843(8): p. 1578-1591.

65. Deng., H., et al., Strategies of exploitation of mammalian reservoirs by Bartonella species. Veterinary research, 2012. 43(1): p. 15-15.

66. Clough., B., et al., Mechanism of regulation of malarial invasion by extraerythrocytic ligands. Molecular and Biochemical Parasitology, 1995. 69(1): p. 19-27.

67. Deng., H.K., et al., Identification of Bartonella Trw host-specific receptor on erythrocytes. PLOS one, 2012. 7(7): p. e41447-e41447.

68. Okoye., V.C. et al., Plasmodium falciparum malaria: band 3 as a possible receptor during invasion of human erythrocytes. Science, 1985. 227(4683): p. 169-171.

69. Padmalayam., I., et al., The gene encoding the 17-kDa antigen of Bartonella henselae is located within a cluster of genes homologous to the virB virulence operon. DNA and Cell Biology, 2000. 19(6): p. 377-382.

70. Sweger., D., et al., Conservation of the 17-kilodalton antigen gene within the genus Bartonella. Clinical and Diagnostic Laboratory Immunology, 2000. 7(2): p. 251-257.

71. Chandran Darbari., V. et al., Structural Biology of Bacterial Type IV Secretion Systems. Annual Review of Biochemistry, 2015. 84: p. 603-629.

72. Québatte., M. et al., Bartonella gene transfer agent: Evolution, function, and proposed role in host adaptation. Cellular Microbiology, 2019. 21(11): p. e13068-e13068.

73. Butcher., D., Muller's ratchet, epistasis and mutation effects. Genetics, 1995. 141(1): p. 431-437.

74. Takeuchi., N., et al., Horizontal gene transfer can rescue prokaryotes from Muller's ratchet: benefit of DNA from dead cells and population subdivision. G3 (Bethesda, Md.), 2014. 4(2), 325-339.

75. Québatte., M., et al., Gene Transfer Agent Promotes Evolvability within the Fittest Subpopulation of a Bacterial Pathogen. Cell systems, 2017. 4(6): p. 611-621.

76. Tamarit., D., et al., Origin and Evolution of the Bartonella Gene Transfer Agent. Molecular Bioogy and Evolution, 2018. 35(2): p. 451-464.

77. Abranches., J., et al., The molecular alarmone (p)ppGpp mediates stress responses, vancomycin tolerance, and virulence in Enterococcus faecalis. Journal of Bacteriology, 2009. 191(7): p. 2248-2256.

78. Ronneau., S. et al., Make and break the alarmone: regulation of (p)ppGpp synthetase/hydrolase enzymes in bacteria. FEMS Microbiology Reviews, 2019. 43(4): p. 389-400.

79. Berglund., E.C., et al., Rapid diversification by recombination in Bartonella grahamii from wild rodents in Asia contrasts with low levels of genomic divergence in Northern Europe and America. Molecular Ecology, 2010. 19(11): p. 2241-2255.

80. Gutiérrez., R., et al., Untangling the knots: Co-infection and diversity of Bartonella from wild gerbils and their associated fleas. Molecular Ecology, 2018. 27(23): p. 47874807.

81. Khan., S.R., et al., Broad-host-range expression vectors with tightly regulated promoters and their use to examine the influence of TraR and TraM expression on Ti plasmid quorum sensing. Applied and Environmental Microbiology, 2008. 74(16): p. 5053-5062.

82. Schulein., R., et al., A bipartite signal mediates the transfer of type IV secretion substrates of Bartonella henselae into human cells. Proceedings of the National Academy of Sciences of the United States of America, 2005. 102(3): p. 856-861. 
83. Schröder., G. et al., Virulence-associated type IV secretion systems of Bartonella. Trends in Microbiology, 2005. 13(7): p. 336-342.

84. Pulliainen, A.T., et al., Bacterial effector binds host cell adenylyl cyclase to potentiate Gas-dependent cAMP production. Proceedings of the National Academy of Sciences of the United States of America, 2012. 109(24): p. 9581-9586.

85. Rhomberg., T., et al., A translocated protein of Bartonella henselae interferes with endocytic uptake of individual bacteria and triggers uptake of large bacterial aggregates via the invasome. Cellular Microbiology, 2009. 11(6): p. 927-945.

86. Truttmann., M.C., et al., Combined action of the type IV secretion effector proteins BepC and BepF promotes invasome formation of Bartonella henselae on endothelial and epithelial cells. Cellular Microbiology, 2011. 13(2): p. 284-299.

87. Dehio., C., Bartonella-host-cell interactions and vascular tumour formation. Nature Reviews Microbiology, 2005. 3(8): p. 621-631.

88. Wang., C., et al., Bartonella quintana type IV secretion effector BepE-induced selective autophagy by conjugation with K63 polyubiquitin chain. Cellular Microbiology, 2019. 21(4): p. e12984.

89. Okujava., R., et al., A translocated effector required for Bartonella dissemination from derma to blood safeguards migratory host cells from damage by co-translocated effectors. PLOS Pathogens, 2014. 10(6): p. e1004187-e1004187.

90. Ogawa., M., et al., Escape of intracellular Shigella from autophagy. Science, 2005. 307(5710): p. 727-731.

91. Wagner., A. et al., Role of distinct type-IV-secretion systems and secreted effector sets in host adaptation by pathogenic Bartonella species. Cellular Microbiology, 2019. 21(3): p. e13004-e13004.

92. Schmid., M.C., et al., A translocated bacterial protein protects vascular endothelial cells from apoptosis. PLOS pathogens, 2006. 2(11): p. e115-e115.

93. Woolery., A.R., et al., AMPylation: Something Old is New Again. Frontiers in Microbiology, 2010. 1: p. 113-113.

94. Kustu., S., et al., Covalent modification of bacterial glutamine synthetase: physiological significance. Molecular and General Genetics: $M G G, 1984$. 197(2): p. 309-317.

95. Brown., M.S., et al., Modulation of glutamine synthetase adenylylation and deadenylylation is mediated by metabolic transformation of the P II -regulatory protein. Proceedings of the National Academy of Sciences of the United States of America, 1971. 68(12): p. 2949-2953.

96. Yarbrough., M.L., et al., AMPylation of Rho GTPases by Vibrio VopS Disrupts Effector Binding and Downstream Signaling. Science, 2009. 323(5911): p. 269-272.

97. Worby., C.A., et al., The fic domain: regulation of cell signaling by adenylylation. Molecular Cell, 2009. 34(1): p. 93-103.

98. Roy., C.R. et al., Bacterial FIC Proteins AMP Up Infection. Science Signaling, 2009. 2(62): pe14.

99. Hodge., R.G., et al., Regulating Rho GTPases and their regulators. Nature Reviews Molecular Cell Biology, 2016. 17(8): p. 496-510.

100. Luong., P., et al., Kinetic and structural insights into the mechanism of AMPylation by VopS Fic domain. Journal of Biological Chemistry, 2010. 285(26): p. 20155-20163.

101. Müller., M.P., et al., The Legionella effector protein DrrA AMPylates the membrane traffic regulator Rab1b. Science, 2010. 329(5994): p. 946-949.

102. Palanivelu., D.V., et al., Fic domain-catalyzed adenylylation: insight provided by the structural analysis of the type IV secretion system effector BepA. Protein science: a publication of the Protein Society, 2011. 20(3): p. 492-499. 
103. Pieles., K., et al., An experimental strategy for the identification of AMPylation targets from complex protein samples. Proteomics, 2014. 14(9): p. 1048-1052.

104. Lewallen., D.M., et al., Probing adenylation: using a fluorescently labelled ATP probe to directly label and immunoprecipitate VopS substrates. Molecular BioSystems, 2012. 8(6): p. 1701-1706.

105. Hao., Y.-H., et al., Characterization of a rabbit polyclonal antibody against threonineAMPylation. Journal of Biotechnology, 2011. 151(3): p. 251-254.

106. Eriksson., J.E., et al., Introducing intermediate filaments: from discovery to disease. The Journal of Clinical Investigation, 2009. 119(7): p. 1763-1771.

107. Wagner., A., et al., Versatility of the BID Domain: Conserved Function as Type-IVSecretion-Signal and Secondarily Evolved Effector Functions Within BartonellaInfected Host Cells. Frontiers in Microbiology, 2019. 10(921).

108. Stanger., F.V., et al., The BID Domain of Type IV Secretion Substrates Forms a Conserved Four-Helix Bundle Topped with a Hook. Structure (London, England : 1993), 2017. 25(1): p. 203-211.

109. Seeger., T.S., et al., Myozap, a novel intercalated disc protein, activates serum response factor-dependent signaling and is required to maintain cardiac function in vivo. Circulation Research, 2010. 106(5): p. 880-890.

110. Bishop., A.L. et al., Rho GTPases and their effector proteins. The Biochemical Journal, 2000. 348 Pt 2(Pt 2): p. 241-255.

111. Kim., J.G., et al., Regulation of RhoA GTPase and various transcription factors in the RhoA pathway. J Cellular Physiology, 2018. 233(9): p. 6381-6392.

112. Hoang., T.T., et al., A broad-host-range Flp-FRT recombination system for site-specific excision of chromosomally-located DNA sequences: application for isolation of unmarked Pseudomonas aeruginosa mutants. Gene, 1998. 212(1): p. 77-86.

113. Lambert., C., Investigation of Bartonella quintana Host Immune Evasion : a thesis submitted to the Victoria University of Wellington in fulfilment of the requirements for the degree of Master of Science in Molecular Microbiology. 2018.

114. Little., A.J., Investigation of Immune Modulation by a Bartonella quintana Effector Protein : a thesis submitted to the Victoria University of Wellington in fulfilment of the requirements for the degree of Master of Science in Molecular Microbiology. 2016.

115. Untergasser., A., et al., Primer3--new capabilities and interfaces. Nucleic acids research, 2012. 40(15): p. e115.

116. Du., K.L., et al., Myocardin Is a Critical Serum Response Factor Cofactor in the Transcriptional Program Regulating Smooth Muscle Cell Differentiation. Molecular and Cellular Biology, 2003. 23(7): p. 2425-2437.

117. Raskatov., J.A., et al., Modulation of $N F-\kappa B$-dependent gene transcription using programmable DNA minor groove binders. Proceedings of the National Academy of Sciences, 2012. 109(4): p. 1023-1028.

118. Fernández-González., E., et al., Transfer of R388 derivatives by a pathogenesisassociated type IV secretion system into both bacteria and human cells. Journal of Bacteriology, 2011. 193(22): p. 6257-6265.

119. Freed., N.E., Creation of a Dense Transposon Insertion Library Using Bacterial Conjugation in Enterobacterial Strains Such As Escherichia Coli or Shigella flexneri. Journal of visualized experiments : JoVE, 2017 (127): p. 56216.

120. Ramesh., G., et al., Cytokines and chemokines at the crossroads of neuroinflammation, neurodegeneration, and neuropathic pain. Mediators of Inflammation, 2013. 2013: p. 480739-480739.

121. Semenova., N., et al., Multiple cytosolic DNA sensors bind plasmid DNA after transfection. Nucleic Acids Research, 2019. 47(19): p. 10235-10246. 
122. Holbrook., J., et al., Tumour necrosis factor signalling in health and disease. F1000Research, 2019. 8: p. F1000 Faculty Rev-1111.

123. Mogensen., T.H., Pathogen Recognition and Inflammatory Signaling in Innate Immune Defenses. Clinical microbiology reviews, 2009. 22(2): p. 240-273.

124. Cornejo., E., et al., How to rewire the host cell: A home improvement guide for intracellular bacteria. Journal of Cell Biology, 2017. 216(12): p. 3931-3948.

125. Capo., C., et al., Bartonella quintana Bacteremia and Overproduction of Interleukin10: Model of Bacterial Persistence in Homeless People. The Journal of Infectious Diseases, 2003. 187(5): p. 837-844.

126. Ouyang., W. et al., IL-10 Family Cytokines IL-10 and IL-22: from Basic Science to Clinical Translation. Immunity, 2019. 50(4): p. 871-891.

127. Gabryšová., L., et al., The Regulation of IL-10 Expression, in Interleukin-10 in Health and Disease. Current topics in Microbiology and Immunology, 380, p. 157-190.

128. Deshmane., S.L., et al., Monocyte chemoattractant protein-1 (MCP-1): an overview. Journal of Interferon \& Cytokine Research: The official journal of the International Society for Interferon and Cytokine Research, 2009. 29(6): p. 313-326.

129. Siddiqui., J.A. et al., CCL2/Monocyte Chemoattractant Protein 1 and Parathyroid Hormone Action on Bone. Frontiers in Endocrinology, 2017. 8(49).

130. Gschwandtner., M., et al., More Than Just Attractive: How CCL2 Influences Myeloid Cell Behavior Beyond Chemotaxis. Frontiers in Immunology, 2019. 10(2759).

131. Vazirinejad., R., et al., The Biological Functions, Structure and Sources of CXCL10 and Its Outstanding Part in the Pathophysiology of Multiple Sclerosis. Neuroimmunomodulation, 2014. 21(6): p. 322-330.

132. Soria., G., et al., The inflammatory chemokines CCL2 and CCL5 in breast cancer. Cancer letters, 2008. 267(2): p. 271-285.

133. Kasama., T., et al., Macrophage Migration Inhibitory Factor: A Multifunctional Cytokine in Rheumatic Diseases. Arthritis, 2010. 2010: p. 106202.

134. Zhang., Z.J., et al., Chemokine contribution to neuropathic pain: respective induction of CXCL1 and CXCR2 in spinal cord astrocytes and neurons. Pain, 2013. 154(10): p. 2185-2197.

135. Russo., R.C., et al., The CXCL8/IL-8 chemokine family and its receptors in inflammatory diseases. Expert Review of Clinical Immunology, 2014. 10(5): p. 593619.

136. Rosales., C., Neutrophil: A Cell with Many Roles in Inflammation or Several Cell Types? Frontiers in Physiology, 2018. 9: p. 113.

137. Tanaka., T., et al., IL-6 in inflammation, immunity, and disease. Cold Spring Harbor perspectives in Biology, 2014. 6(10): p. a016295-a016295.

138. Yang., S., et al., Role of TNF-TNF Receptor 2 Signal in Regulatory T Cells and Its Therapeutic Implications. Frontiers in Immunology, 2018. 9(784).

139. Zelová., H., et al., TNF- $\alpha$ signalling and inflammation: interactions between old acquaintances. Inflammation Research, 2013. 62(7): p. 641-651.

140. Dalby., B., et al., Advanced transfection with Lipofectamine 2000 reagent: primary neurons, siRNA, and high-throughput applications. Methods, 2004. 33(2): p. 95-103.

141. Deepak., S.A., et al., Real-Time PCR: Revolutionizing Detection and Expression Analysis of Genes. Current genomics, 2007. 8(4): p. 234-251.

142. Kuang., J., et al., An overview of technical considerations when using quantitative realtime PCR analysis of gene expression in human exercise research. PLOS one, 2018. 13(5): p. e0196438-e0196438.

143. Bustin., S.A., et al., Quantitative real-time RT-PCR--a perspective. Journal of Molecular Endocrinology, 2005. 34(3): p. 597-601. 
144. San Segundo-Val., I. et al., Introduction to the Gene Expression Analysis, in Molecular Genetics of Asthma. Springer 2016 (New York: New York, NY). p. 29-43.

145. Nigro., P., et al., Cyclophilin A: a key player for human disease. Cell Death \& Disease, 2013. 4(10): p. e888-e888.

146. Lin., J. et al., Histological evidence: housekeeping genes beta-actin and GAPDH are of limited value for normalization of gene expression. Development Genes and Evolution, 2012. 222(6): p. 369-376.

147. Bosnjak., M., et al., Inhibition of the Innate Immune Receptors for Foreign DNA Sensing Improves Transfection Efficiency of Gene Electrotransfer in Melanoma B16F10 Cells. The Journal of Membrane Biology, 2018. 251(2): p. 179-185.

148. Li., Y., et al., Characterization of AMPylation on threonine, serine, and tyrosine using mass spectrometry. Journal of the American Society for Mass Spectrometry, 2011. 22(4): p. 752-761.

149. Petinaki., E., et al., Lincomycin resistance gene $\operatorname{lnu}(D)$ in Streptococcus uberis. Antimicrobial agents and Chemotherapy, 2008. 52(2): p. 626-630.

150. Mehta., R., et al., Adenylylation and catalytic properties of Mycobacterium tuberculosis glutamine synthetase expressed in Escherichia coli versus mycobacteria. Journal of Biological Chemistry, 2004. 279(21): p. 22477-22482.

151. Alsmark., C.M., et al., The louse-borne human pathogen Bartonella quintana is a genomic derivative of the zoonotic agent Bartonella henselae. Proceedings of the National Academy of Sciences of the United States of America, 2004. 101(26): p. 97169721.

152. Liu., Y., et al., SacB-SacR Gene Cassette As the Negative Selection Marker to Suppress Agrobacterium Overgrowth in Agrobacterium-Mediated Plant Transformation. Frontiers in Molecular Biosciences, 2016. 3(70).

153. Grasseschi., H.A.et al., Transformation of Bartonella bacilliformis by electroporation. Canadian Journal of Microbiology, 1994. 40(9): p. 782-786.

154. Wang., Y., et al., Development of a Transformation System for Chlamydia trachomatis: Restoration of Glycogen Biosynthesis by Acquisition of a Plasmid Shuttle Vector. PLOS Pathogens, 2011. 7(9): p. e1002258.

155. Riess., T., et al., Bartonella adhesin a mediates a proangiogenic host cell response. The Journal of experimental medicine, 2004. 200(10): p. 1267-1278.

156. Potter., H., Transfection by electroporation. Current protocols in Molecular Biology, 2003. Chapter 9: p. Unit-9.3.

157. Luft., C. et al., Electroporation Knows No Boundaries: The Use of Electrostimulation for siRNA Delivery in Cells and Tissues. Journal of Biomolecular Screening, 2015. 20(8): p. 932-942.

158. Wise., A.A., et al., Three Methods for the Introduction of Foreign DNA into Agrobacterium, in Agrobacterium Protocols. 2006, Humana Press: Totowa, NJ. p. 4354.

159. Dehio., C., et al., Construction of versatile high-level expression vectors for Bartonella henselae and the use of green fluorescent protein as a new expression marker. Gene, 1998. 215(2): p. 223-229.

160. Seubert., A., et al., A bacterial conjugation machinery recruited for pathogenesis. Molecular Microbiology, 2003. 49(5): p. 1253-1266.

161. Heinaru., E., et al., Conjugal transfer and mobilization capacity of the completely sequenced naphthalene plasmid pNAH20 from multiplasmid strain Pseudomonas fluorescens PC20. FEMS Microbiology Ecology, 2009. 70(3): p. 563-574. 
162. Heinze., S., et al., Transmating: conjugative transfer of a new broad host range expression vector to various Bacillus species using a single protocol. BMC Microbiology, 2018. 18(1): p. 56-56.

163. Carter., M., et al., Chapter 15 - Biochemical Assays and Intracellular Signaling, in Guide to Research Techniques in Neuroscience (Second Edition), 2015, Academic Press: San Diego. p. 311-343.

164. Liu., T., et al., NF- $\kappa B$ signaling in inflammation. Signal Transduction and Targeted Therapy, 2017. 2(1): p. 17023.

165. Sun., S.C., et al., Regulation of nuclear factor- $\kappa B$ in autoimmunity. Trends in Immunology, 2013. 34(6): p. 282-289.

166. Nie., L., et al., Identification of transcription factor-DNA interactions using chromatin immunoprecipitation assays. Methods in Molecular Biology (Clifton, N.J.), 2009. 493: p. 311-321.

167. Mondeel., T., et al., ChIP-exo analysis highlights Fkh1 and Fkh2 transcription factors as hubs that integrate multi-scale networks in budding yeast. Nucleic Acids Research, 2019. 47(15): p. 7825-7841.

168. Euskirchen., G.M., et al., Mapping of transcription factor binding regions in mammalian cells by ChIP: comparison of array-and sequencing-based technologies. Genome Research, 2007. 17(6): p. 898-909.

169. Roca., H. et al., Analysis of transcription factor interactions in osteoblasts using competitive chromatin immunoprecipitation. Nucleic Acids Research, 2008. 36(5): p. 1723-1730.

170. Safgren., S.L., et al., The transcription factor GLI1 cooperates with the chromatin remodeler SMARCA2 to regulate chromatin accessibility at distal DNA regulatory elements. Journal of Biological Chemistry, 2020. 295(26): p. 8725-8735.

171. Hayashi., K., et al., A novel inhibitory mechanism of MRTF-A/B on the ICAM-1 gene expression in vascular endothelial cells. Scientific Reports, 2015. 5(1): p. 10627.

172. White., R.L., What In Vitro Models of Infection Can and Cannot Do. Pharmacotherapy: The Journal of Human Pharmacology and Drug Therapy, 2001. 21(11P2): p. 292S$301 \mathrm{~S}$. 


\subsection{Pairwise alignment of BepA1 WT protein and the BepA1 FIC domain mutant DNA sequence.}

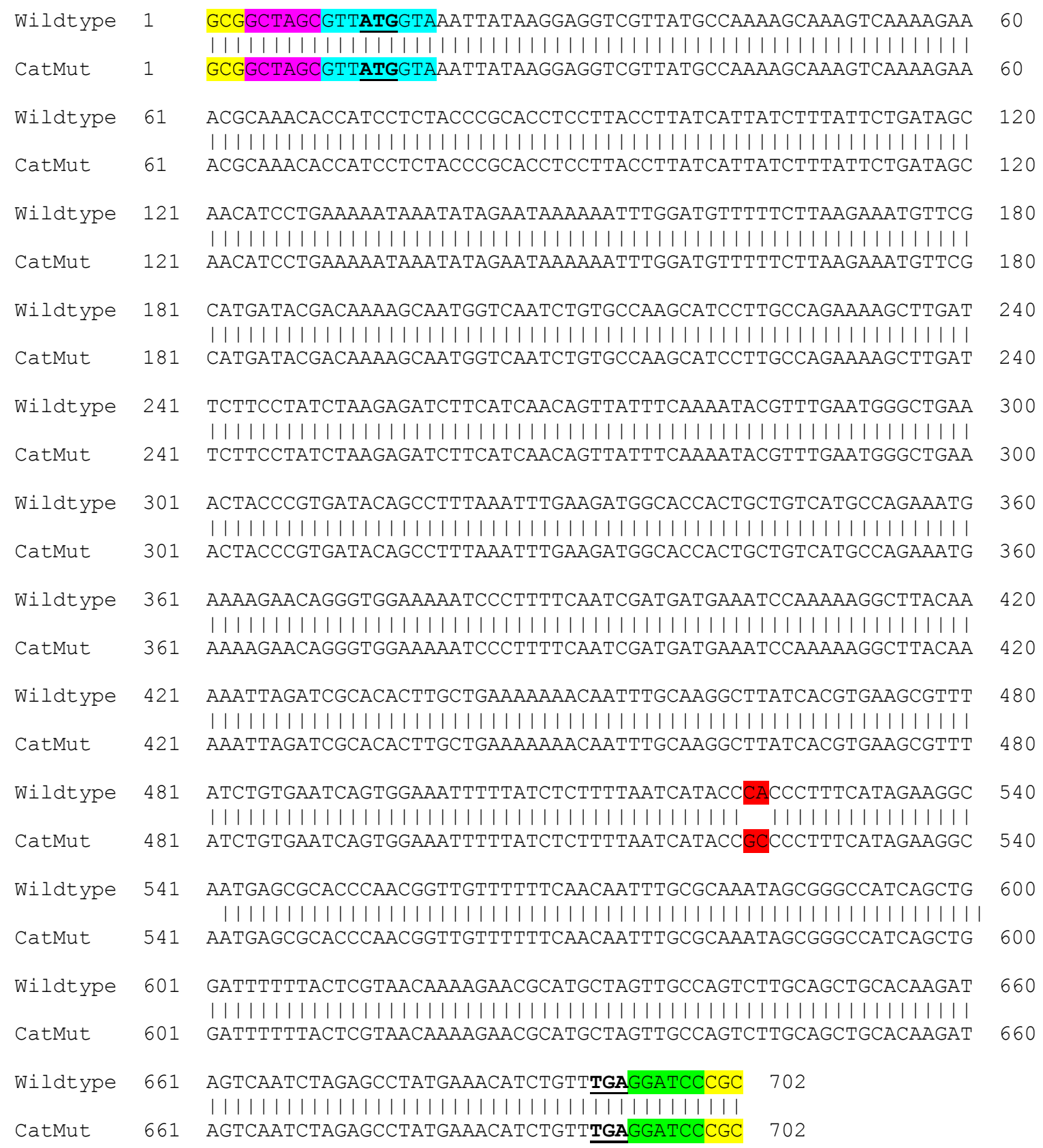

\section{Appendix Figure 7.1 Sequence alignment for the BepA1 WT protein and the BepA1 FIC domain}

mutant. Pink and green highlights represent the NheI and BamHI restriction sites respectively. The red highlight represents the mutated bases that change a histidine to an alanine. The blue highlight is the Kozak sequence to allow for the initiation of protein translation in eukaryotes. Yellow highlight are spacer regions (excess DNA to facilitate restriction digest). ATG start and TGA stop codons are in bold and underlined. 
7.2 Pairwise alignment of BepA1 WT protein and the BepA1 FIC domain mutant protein amino acid sequence.

\begin{tabular}{|c|c|c|}
\hline Wildtype & 1 & $\begin{array}{l}\text { MVNYKEVVMPKAKSKETQTPSSTRTSLPYHYLYSDSNILKNKYRIKNLDVFLKKCSHDTT } \\
\text { MVNYKEVVMPKAKSKETQTPSSTRTSLPYHYLYSDSNILKNKYRIKNLDVFLKKCSHDTT }\end{array}$ \\
\hline CatMut & 1 & MVNYKEVVMPKAKSKETQTPSSTRTSLPYHYLYSDSNILKNKYRIKNLDVFLKKCSHDTT \\
\hline Wildtype & 61 & $\begin{array}{l}\text { KAMVNLCQASLPEKLDSSYLRDLHQQLFQNTFEWAETTRDTAFKFEDGTTAVMPEMKRTG } \\
\text { KAMVNLCQASLPEKLDSSYLRDLHQQLFQNTFEWAETTRDTAFKFEDGTTAVMPEMKRTG }\end{array}$ \\
\hline CatMut & 61 & KAMVNLCQASLPEKLDSSYLRDLHQQLFQNTFEWAETTRDTAFKFEDGTTAVMPEMKRTG \\
\hline CatMut & 121 & WKNPFS IDDEIQKGLQKLDRTLAEKNNLQGLSREAFICESVEIFISFNHTAPFIEGNERT \\
\hline Wildtype & 181 & $\begin{array}{l}\text { QRLFFQQFAQIAGHQLDFLLVTKERMLVASLAAAQDSQSRAYETSV } \\
\text { QRLFFQQFAQIAGHQLDFLLVTKERMLVASLAAAQDSQSRAYETSV }\end{array}$ \\
\hline
\end{tabular}

Appendix Figure 7.2 Predicted amino acid sequence alignment for the BepA1 WT protein and the BepA1 FIC domain mutant. The sequences represent the amino acids in the BepA1 WT protein and the BepA1 FIC domain mutant protein. The BepA1 WT protein encodes the invariant Histidine, proven essential for AMPylation function, seen in the blue highlight. The BepA1 FIC domain mutant contains a point mutant where the histidine has been changed to an alanine, represented by the pink highlight, a mutation thought to abolish this AMPylation function. 


\section{Human Cytokine Array Coordinates}

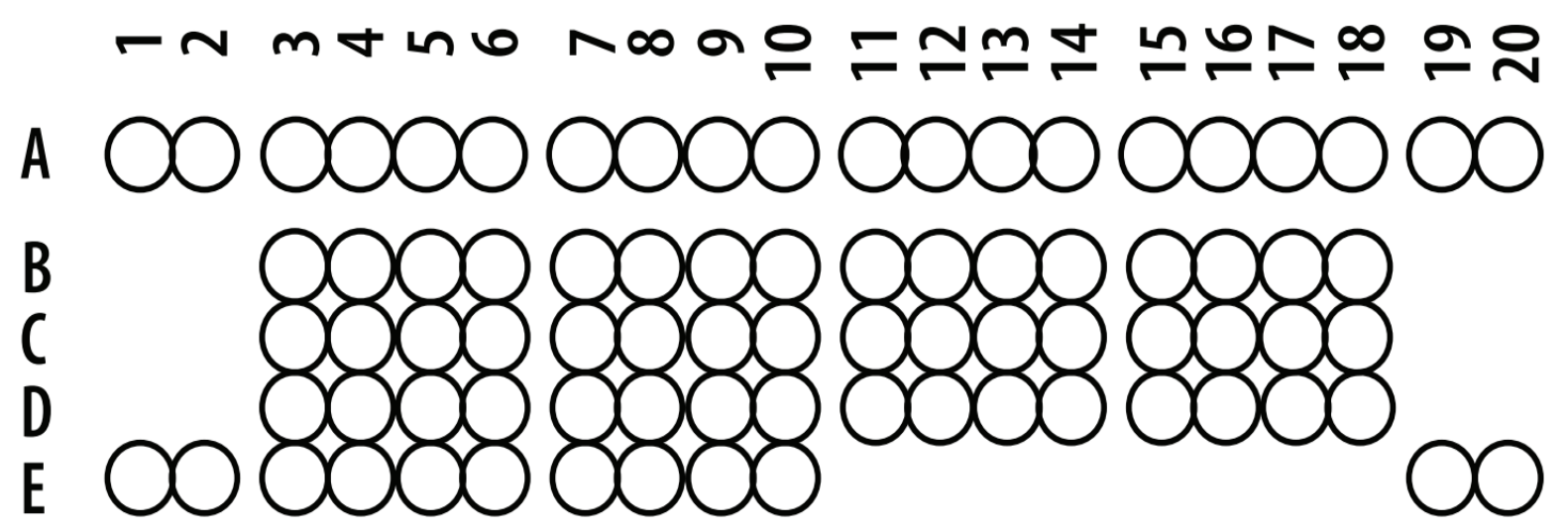

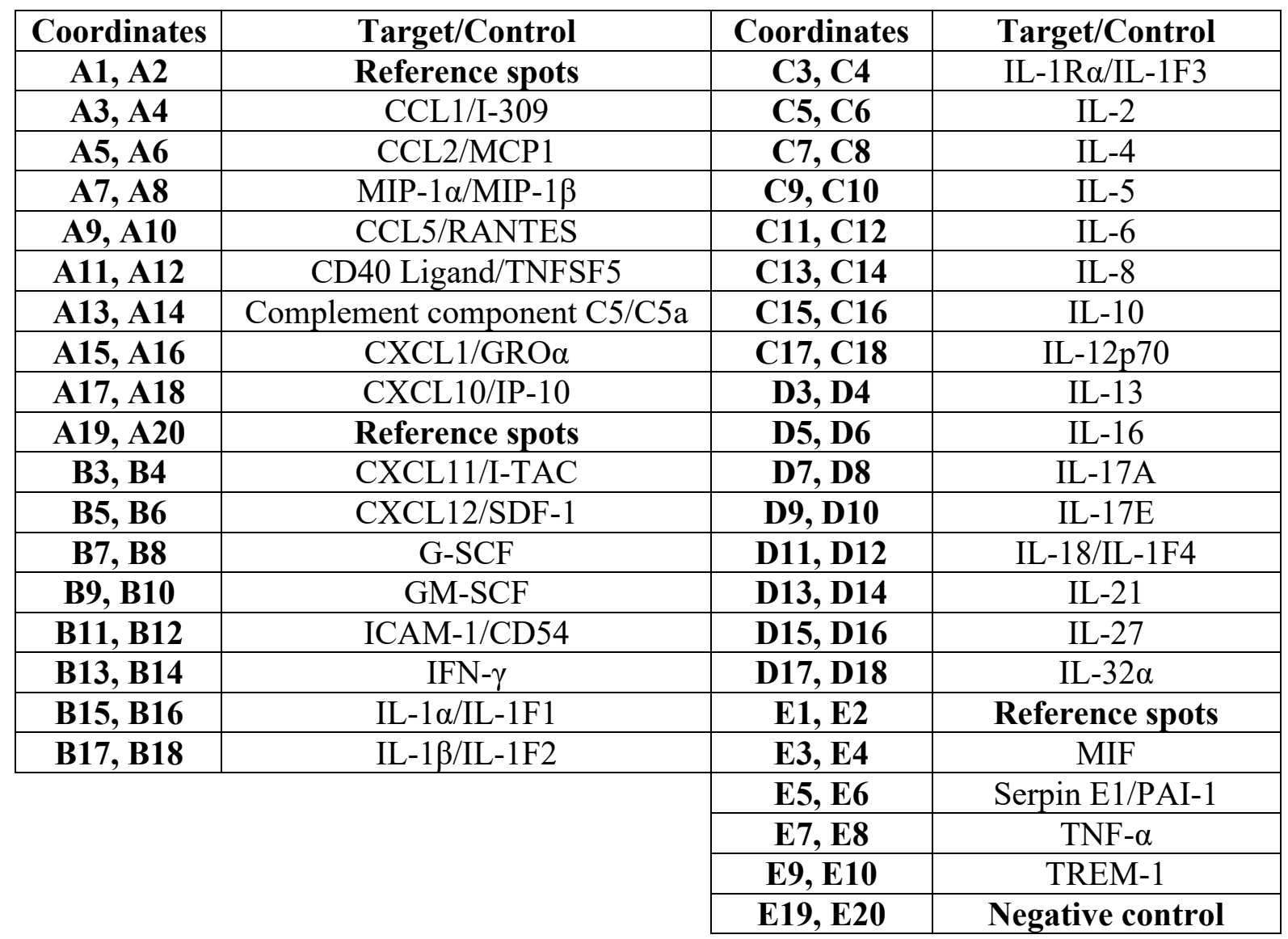

Appendix Figure 7.3 Proteome Profiler Human Cytokine Array Membrane Coordinates. Top: each membrane of the Proteome Profiler cytokine array kit was spotted with capture antibodies in duplicate. Image shows the coordinate of each cytokine, chemokine and serine protease antibodies on the membrane. Bottom table: shows the cytokine/chemokine/serine protease target, or control of each target, and reference spots. 


\section{Cell mediums Cell lysates}

HeLa untransfected, no TNF- $\alpha$ treatment:
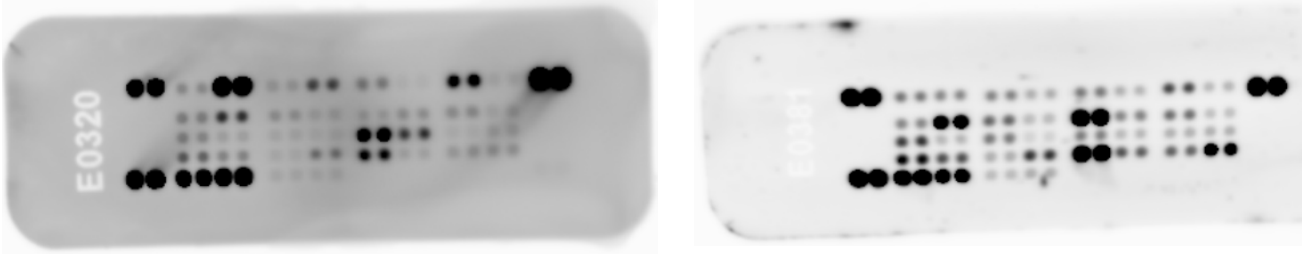

$\underline{\text { HeLa untransfected, TNF- } \alpha \text { treated: }}$
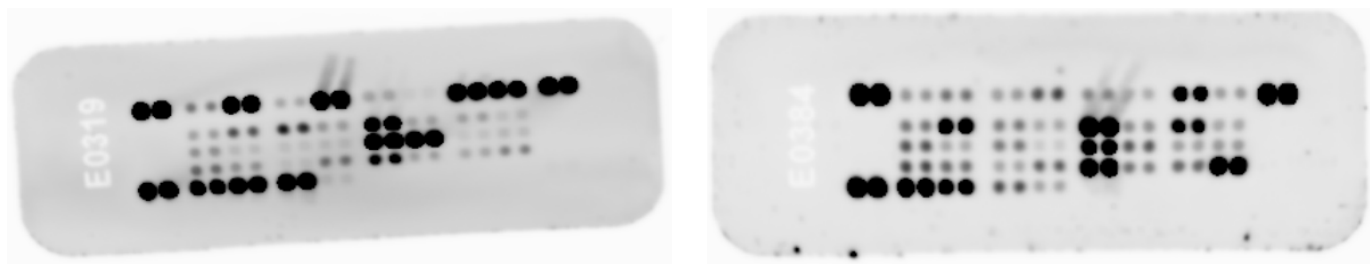

BepA1 WT transfected, TNF- $\alpha$ treated:
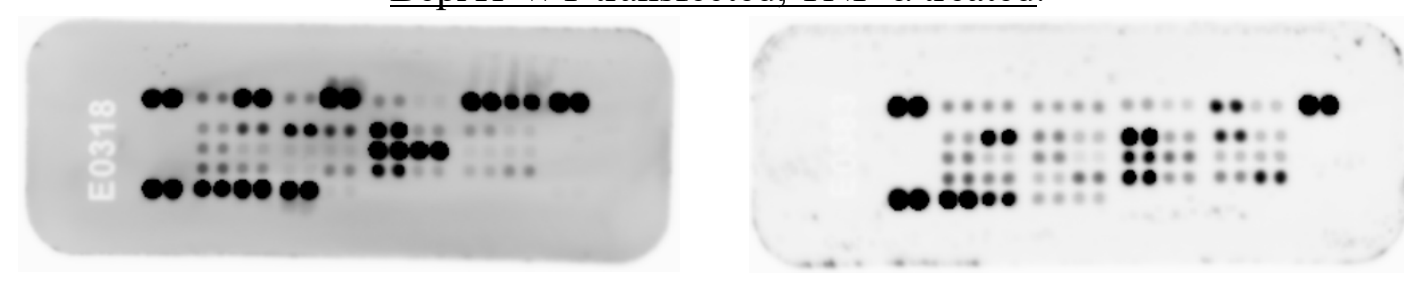

$\underline{\text { FIC domain mutant transfected, TNF- } \alpha \text { treated: }}$
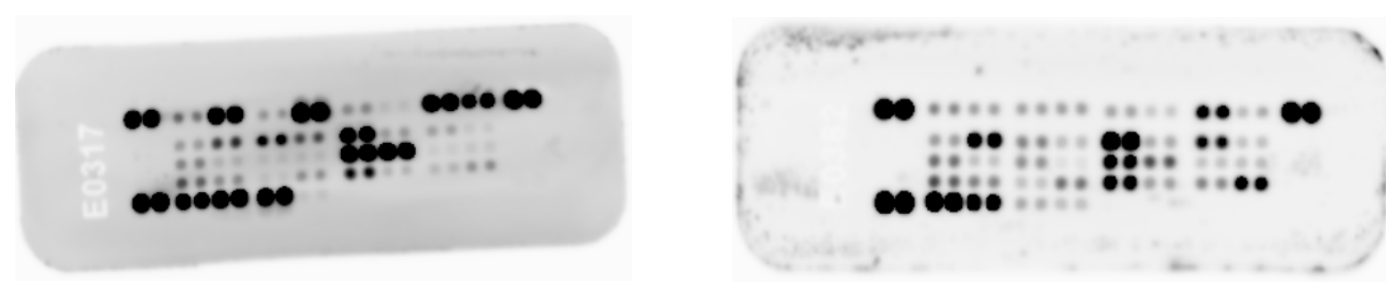

Appendix Figure 7.4 Proteome profiler Exposed Membranes for Cell mediums and Cell lysates. Each membrane shows the capture antibodies bound by the cytokine/chemokine/serine protease target for all four HeLa cell samples.

Each membrane was exposed for four minutes in the Amersham Imager 600 before images were recorded. Differences can be noted between the spotting patterns between samples, demonstrating the different targets bound and the different intensities. 
7.5 Average pixel intensity values for HeLa cell mediums samples

\begin{tabular}{|c|c|c|c|c|}
\hline Target/control & HeLa basal & $\begin{array}{c}\text { HeLa } \\
\text { untransfected } \\
\text { TNF- } \alpha \text { treated }\end{array}$ & $\begin{array}{c}\text { BepA1 WT } \\
\text { transfected, } \\
\text { TNF- } \alpha \text { treated }\end{array}$ & $\begin{array}{c}\text { FIC domain } \\
\text { mutant } \\
\text { transfected, } \\
\text { TNF- } \alpha \text { treated }\end{array}$ \\
\hline CCL1/I-309 & 4436.5 & 2729 & 9730 & 6026.5 \\
\hline CCL2/MCP-1 & 20755.5 & 24273.5 & 26661.5 & 33893.5 \\
\hline 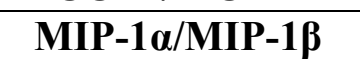 & 5158 & 887 & 8524.5 & 4997.5 \\
\hline CCL5/RANTES & 8329 & 28692 & 34755 & 43232 \\
\hline $\begin{array}{c}\text { CD40 } \\
\text { Ligand/TNFSF5 }\end{array}$ & 4971 & 3130.5 & 7957 & 7018 \\
\hline $\begin{array}{c}\text { Complement } \\
\text { component } \mathrm{C} 5 / \mathrm{C} 5 \mathrm{a}\end{array}$ & 620.5 & 745 & 4480.5 & 3447.5 \\
\hline CXCL/GROa & 10287 & 32066 & 29344 & 37888.5 \\
\hline CXCL 10/IP-10 & 4627 & 29615 & 24754 & 30212 \\
\hline CXCL11/I-TAC & 4325 & 939 & 5330.5 & 3325.5 \\
\hline CXCL 12/SDF-1 & 6007.5 & 3772 & 9682.5 & 10779 \\
\hline G-CSF & 4658.5 & 7503.5 & 17463 & 17726 \\
\hline GM-CSF & 6125 & 3546 & 14303.5 & 12266.5 \\
\hline ICAM-1/CD54 & 5626 & 25124 & 37145.5 & 33756.5 \\
\hline IFN- $\gamma$ & 3574.5 & 8127.5 & 7454 & 7276 \\
\hline IL-1 $\alpha /$ IL-1F1 & 3626 & 2729.5 & 4636 & 3184.5 \\
\hline IL-1及/IL-1F2 & 6061.5 & 2020.5 & 2446.5 & 645 \\
\hline IL-1ra/IL-1F3 & 4971 & 3356.5 & 6696 & 5524 \\
\hline IL-2 & 2265.5 & 1907 & 5530 & 3832 \\
\hline IL-4 & 3274.5 & 2548 & 7683.5 & 4944 \\
\hline IL-5 & 5937.5 & 4433.5 & 8498.5 & 5047 \\
\hline IL-6 & 19065.5 & 33004 & 32658 & 39804 \\
\hline IL-8 & 8341.5 & 29732.5 & 28289 & 37557 \\
\hline IL-10 & 2810 & 1228 & 1240.5 & -185 \\
\hline IL-12p70 & 6194.5 & 1428.5 & 1265 & 387.5 \\
\hline IL-13 & 6209.5 & 7845 & 9942.5 & 10498 \\
\hline IL-16 & 2957 & 5229 & 7267 & 5910.5 \\
\hline IL-17A & 2497.5 & 3947 & 5636 & 3313 \\
\hline IL-17E & 7082 & 7720.5 & 7385 & 4271.5 \\
\hline IL-18/IL-1F4 & 12550.5 & 13878 & 14519 & 13329.5 \\
\hline IL-21 & 2752.5 & 3512 & 3362.5 & 1860 \\
\hline IL-27 & 3829.5 & 1705 & 2052 & 1362.5 \\
\hline IL-32a & 4223 & 2387.5 & 3352.5 & 3571.5 \\
\hline MIF & 18856 & 24612 & 25178 & 32653.5 \\
\hline Serpin E1/PAI-1 & 23155.5 & 29938 & 27834.5 & 37433 \\
\hline TNF- $\alpha$ & 2322 & 29825 & 26526.5 & 37441 \\
\hline TREM-1 & 4703.5 & 5434.5 & 3196.5 & 1821.5 \\
\hline CCL1/I-309 & 4436.5 & 2729 & 9730 & 6026.5 \\
\hline
\end{tabular}

Appendix Table 7.5.1 Average pixel intensity values for HeLa cell medium samples. Average background values are displayed. 
7.5 Average pixel intensity values for HeLa cell lysates samples

\begin{tabular}{|c|c|c|c|c|}
\hline Target/control & HeLa basal & $\begin{array}{c}\text { HeLa } \\
\text { untransfected } \\
\text { TNF- } \alpha \text { treated }\end{array}$ & $\begin{array}{c}\text { BepA1 WT } \\
\text { transfected, } \\
\text { TNF- } \alpha \text { treated }\end{array}$ & $\begin{array}{c}\text { FIC domain } \\
\text { mutant } \\
\text { transfected, } \\
\text { TNF- } \alpha \text { treated }\end{array}$ \\
\hline CCL1/I-309 & 4685.5 & 4359 & 9377.5 & 5262.5 \\
\hline CCL2/MCP-1 & 4150 & 7333.5 & 7819 & 3809 \\
\hline MIP-1 $\alpha / M I P-1 \beta$ & 4465 & 4550.5 & 6677.5 & 3937 \\
\hline CCL5/RANTES & 3118 & 11572.5 & 7375.5 & 4239 \\
\hline $\begin{array}{c}\text { CD40 } \\
\text { Ligand/TNFSF5 }\end{array}$ & 6036 & 9530 & 6661 & 3884.5 \\
\hline $\begin{array}{c}\text { Complement } \\
\text { component } \mathrm{C} 5 / \mathrm{C} 5 \mathrm{a}\end{array}$ & 2559 & 4369 & 2482 & 1656.5 \\
\hline CXCL/GROa & 5548.5 & 29940.5 & 25907 & 20398 \\
\hline CXCL 10/IP-10 & 2281 & 3991 & 3077 & 2799.5 \\
\hline CXCL11/I-TAC & 5608.5 & 6778 & 8058.5 & 5245.5 \\
\hline CXCL 12/SDF-1 & 28452.5 & 33542 & 45326.5 & 26618.5 \\
\hline G-CSF & 8852 & 6620.5 & 11099 & 6677 \\
\hline GM-CSF & 3653 & 3253.5 & 4847.5 & 2866.5 \\
\hline ICAM-1/CD54 & 40064.5 & 49038 & 52130.5 & 41229 \\
\hline IFN- $\gamma$ & 6608.5 & 5545.5 & 7300 & 4269.5 \\
\hline IL-1 $\alpha /$ IL-1F1 & 7288 & 26076 & 22006.5 & 16497 \\
\hline IL-1及/IL-1F2 & 2383 & 3151 & 3455 & 2116 \\
\hline IL-1ra/IL-1F3 & 11891.5 & 9632 & 12233.5 & 10241 \\
\hline IL-2 & 4361 & 3621 & 4336 & 3588.5 \\
\hline IL-4 & 7529 & 6694 & 10240 & 7587.5 \\
\hline IL-5 & 2803.5 & 658.5 & 29969.5 & 1191 \\
\hline IL-6 & 13417.5 & 37711 & 12544.5 & 32105 \\
\hline IL-8 & 5392 & 9256 & 3886.5 & 13512 \\
\hline IL-10 & 6052.5 & 4251.5 & 3928 & 3286 \\
\hline IL-12p70 & 6071.5 & 5686 & 2822.5 & 3798 \\
\hline IL-13 & 25681 & 11311 & 16253 & 12955 \\
\hline IL-16 & 17287.5 & 8503 & 8665 & 8739 \\
\hline IL-17A & 6879.5 & 3018.5 & 3154.5 & 3355.5 \\
\hline IL-17E & 6879.5 & 7894 & 9913 & 9992.5 \\
\hline IL-18/IL-1F4 & 55950.5 & 44871 & 30709 & 48331.5 \\
\hline IL-21 & 16243 & 279 & 5905.5 & 10812.5 \\
\hline IL-27 & 14631.5 & 8883.5 & 7129 & 7812.5 \\
\hline IL-32 $\alpha$ & 38336 & 43665.5 & 18290.5 & 27477 \\
\hline MIF & 55819 & 42678 & 47661.5 & 50034.5 \\
\hline Serpin E1/PAI-1 & 46846 & 33637 & 29480 & 37007 \\
\hline TNF-a & 5854.5 & 9765.5 & 4270 & 5022 \\
\hline TREM-1 & 8472.5 & 2908.5 & 3035.5 & 3596 \\
\hline CCL1/I-309 & 4685.5 & 4359 & 9377.5 & 5262.5 \\
\hline
\end{tabular}

Appendix Table 7.5.2 Average pixel intensity values for HeLa cell lysates samples. Average background values are displayed. 
Appendix figures 7.6.1 - 7.6.3 present the raw qPCR data Livak analysis calculations for target genes IL-6, IL-8 and SM22 respectively. To test for any gene expression changes, the Livak Method of analysis was used. Briefly, the detected $\mathrm{C}_{\mathrm{q}}$ (fluorescence) values are summarised for all HeLa cells samples tested for changes in IL-6, IL8 and SM22 gene expression individually. There are three steps in the Livak method of analysis: first, we normalise the $\mathrm{Cq}$ of the target gene to the $\mathrm{Cq}$ of the reference gene both the test sample and the calibrator samples using the formulae:

$$
\begin{aligned}
& \Delta \mathrm{Cq}(\text { test })=\mathrm{Cq}(\text { target, test })-\mathrm{Cq}(\text { ref, test }) \\
& \Delta \mathrm{Cq}(\text { calibrator })=\mathrm{Cq}(\text { target, calibrator })-\mathrm{Cq}(\text { ref, calibrator })
\end{aligned}
$$

Then we normalise the $\Delta \mathrm{Cq}$ of the test sample to the $\Delta \mathrm{Cq}$ of the calibrator using the formula:

$$
\Delta \Delta \mathrm{Cq}=\Delta \mathrm{Cq}(\text { test })-\Delta \mathrm{Cq}(\text { calibrator })
$$

And lastly, we calculated the normalised expression ratio using the formula $\mathbf{2}^{-\mathbf{\Delta} \Delta \mathrm{Cq}}$ which is simply 2 to the negative power of the $\Delta \Delta \mathrm{Cq}$ calculated for each test sample. The calculations for target genes IL-6, IL-8 and SM22 are summarised below. 
IL-6

\begin{tabular}{|c|c|c|c|c|c|c|c|c|c|c|c|c|c|c|c|c|c|c|}
\hline Well & Fluor & Target & Content & Sample & $\mathrm{Cq}$ & Mean Cq & Well & Fluor & Target & Content & Sample & $\mathrm{Cq}$ & Mean Cq & $\begin{array}{l}\text { Normalising } \\
\text { against } \\
\text { reference gene }\end{array}$ & average & $\begin{array}{c}\text { Normalising } \\
\text { against control }\end{array}$ & $\begin{array}{l}2^{\wedge}-(\text { colum } R \text { ) } \\
\text { (Fold change) }\end{array}$ & $\begin{array}{c}\text { Fold } \\
\text { change } \\
\text { average }\end{array}$ \\
\hline $\mathrm{A} 01$ & SYBR & CyclA & Unkn & HeLa basal & $19.35^{\prime}$ & & E01 & SYBR & IL-6 & Unkn & HeLa basal & $N / A$ & & & & & & \\
\hline B01 & SYBR & CycIA & Unkn & HeLa basal & 18.34 & & F01 & SYBR & IL-6 & Unkn & HeLa basal & 27.86 & & 9.519366 & & 0.00 & 1.00 & \\
\hline $\mathrm{C} 01$ & SYBR & CyclA & Unkn & HeLa basal & 18.61 & 18.77 & G01 & SYBR & IL-6 & Unkn & HeLa basal & $\mathrm{N} / \mathrm{A}$ & 27.86 & & 9.519365797 & -9.52 & & \\
\hline A02 & SYBR & CyclA & Unkn & HeLa TNF-a & $18.02^{\prime}$ & & E02 & SYBR & IL-6 & Unkn & HeLa TNF-a & 21.63 & & 3.613321 & & -5.91 & 59.96 & \\
\hline B02 & SYBR & CycIA & Unkn & HeLa TNF-a & 18.25 & & F02 & SYBR & IL-6 & Unkn & HeLa TNF-a & 21.22 & & 2.966197 & & -6.55 & 93.91 & \\
\hline $\mathrm{C} 02$ & SYBR & CycIA & Unkn & HeLa TNF-a & 18.08 & 18.12 & G02 & SYBR & IL-6 & Unkn & HeLa TNF-a & 20.9 & 21.25 & 2.823165 & 3.134227548 & -6.70 & 103.69 & 85.86 \\
\hline $\mathrm{A} 03$ & SYBR & CyclA & Unkn & BepA1 basal & $18.88^{\prime}$ & & E03 & SYBR & IL-6 & Unkn & BepA1 basal & $\mathrm{N} / \mathrm{A}^{\mathrm{T}}$ & & & & -9.52 & & \\
\hline B03 & SYBR & CyclA & Unkn & BepA1 basal & 18.85 & & F03 & SYBR & IL-6 & Unkn & BepA1 basal & 20.23 & & 1.379299 & & -8.14 & 282.10 & \\
\hline $\mathrm{C} 03$ & SYBR & CyclA & Unkn & BepA1 basal & 18.78 & 18.84 & G03 & SYBR & IL-6 & Unkn & BepA1 basal & 20.05 & 20.14 & 1.269544 & 1.324421525 & -8.25 & 304.40 & 293.25 \\
\hline A04 & SYBR & CyclA & Unkn & BepA1 TNF-a & $17.40^{\prime}$ & & E04 & SYBR & IL-6 & Unkn & BepA1 TNF-a & $22.72^{\prime}$ & & 5.316781 & & -4.20 & 18.41 & \\
\hline B04 & SYBR & CycIA & Unkn & BepA1 TNF-a & 17.43 & & F04 & SYBR & IL-6 & Unkn & BepA1 TNF-a & 22.35 & & 4.921624 & & -4.60 & 24.21 & \\
\hline $\mathrm{C} 04$ & SYBR & CycIA & Unkn & BepA1 TNF-a & 17.84 & 17.56 & G04 & SYBR & IL-6 & Unkn & BepA1 TNF-a & 22.11 & 22.39 & 4.268992 & 4.84 & -5.25 & 38.06 & 26.90 \\
\hline A05 & SYBR & CyclA & Unkn & FIC basal & $18.13^{\prime}$ & & E05 & SYBR & IL-6 & Unkn & FIC basal & $25.9^{\prime}$ & & 7.773019 & & -1.75 & 3.36 & \\
\hline B05 & SYBR & CyclA & Unkn & FIC basal & 18.25 & & F05 & SYBR & IL-6 & Unkn & FIC basal & 22.81 & & 4.564559 & & -4.95 & 31.01 & \\
\hline $\mathrm{C} 05$ & SYBR & CyclA & Unkn & FIC basal & 18.38 & 18.25 & G05 & SYBR & IL-6 & Unkn & FIC basal & 22.81 & 23.84 & 4.430221 & 5.589266329 & -5.09 & 34.04 & 22.80 \\
\hline A06 & SYBR & CyclA & Unkn & FIC TNF-a & $18.40^{\prime \prime}$ & & E06 & SYBR & IL-6 & Unkn & FIC TNF-a & $22.02^{\prime}$ & & 3.621397 & & -5.90 & 59.63 & \\
\hline B06 & SYBR & CyclA & Unkn & FIC TNF-a & 18.45 & & F06 & SYBR & IL-6 & Unkn & FIC TNF-a & 20.32 & & 1.872137 & & -7.65 & 200.47 & \\
\hline C06 & SYBR & CycIA & Unkn & FIC TNF-a & 18.58 & 18.48 & G06 & SYBR & IL-6 & Unkn & FIC TNF-a & 20.25 & 20.86 & 1.665858 & 2.39 & -7.85 & 231.28 & 63.79 \\
\hline
\end{tabular}

Appendix figure 7.6.1 qPCR raw data and basic Livak analysis calculations are presented for IL-6. 
IL-8

\begin{tabular}{|c|c|c|c|c|c|c|c|c|c|c|c|c|c|c|c|c|c|c|}
\hline Well & Fluor & Target & Content & Sample & $\mathrm{Cq}$ & $\begin{array}{l}\text { Mean Cq (of } \\
\text { triplicates) }\end{array}$ & Well & Fluor & Target & Content & Sample & $\mathrm{Cq}$ & $\begin{array}{l}\text { Mean Cq (of } \\
\text { triplicates) }\end{array}$ & $\begin{array}{c}\text { Normalising } \\
\text { against } \\
\text { reference } \\
\text { gene }\end{array}$ & average & $\begin{array}{c}\text { Normalising } \\
\text { against control }\end{array}$ & $\begin{array}{l}2^{\wedge}-(\text { colum R) } \\
\text { (Fold change) }\end{array}$ & $\begin{array}{c}\text { Fold } \\
\text { change } \\
\text { average }\end{array}$ \\
\hline A01 & SYBR & CyclA & Unkn & HeLa basal & $19.35^{\prime}$ & & E01 & SYBR & IL-8 & Unkn & HeLa basal & N/A ${ }^{\prime}$ & & & & & & 1.01 \\
\hline B01 & SYBR & CyclA & Unkn & HeLa basal & 18.34 & & F01 & SYBR & IL-8 & Unkn & HeLa basal & 29.11 & & 10.77 & & 0.173 & 0.89 & \\
\hline $\mathrm{C} 01$ & SYBR & CyclA & Unkn & HeLa basal & 18.61 & 18.77 & G01 & SYBR & IL-8 & Unkn & HeLa basal & 29.03 & 29.07 & 10.42 & 10.59647936 & -0.173 & 1.13 & \\
\hline A02 & SYBR & CyclA & Unkn & HeLa TNF-a & $18.02^{\prime}$ & & E02 & SYBR & IL-8 & Unkn & HeLa TNF-a & $21.17^{\prime}$ & & 3.15 & & -7.443 & 174.03 & 196.66 \\
\hline B02 & SYBR & CyclA & Unkn & HeLa TNF-a & 18.25 & & F02 & SYBR & IL-8 & Unkn & HeLa TNF-a & 21.4 & & 3.15 & & -7.450 & 174.89 & \\
\hline $\mathrm{C} 02$ & SYBR & CyclA & Unkn & HeLa TNF-a & 18.08 & 18.12 & G02 & SYBR & IL-8 & Unkn & HeLa TNF-a & 20.76 & 21.11 & 2.68 & 2.99 & -7.913 & 241.07 & \\
\hline $\mathrm{A} 03$ & SYBR & CyclA & Unkn & BepA1 basal & $18.88^{\prime}$ & & E03 & SYBR & IL-8 & Unkn & BepA1 basal & $20.11^{\prime}$ & & $1.23^{\prime}$ & & -9.369 & 661.13 & 574.75 \\
\hline B03 & SYBR & CyclA & Unkn & BepA1 basal & 18.85 & & F03 & SYBR & IL-8 & Unkn & BepA1 basal & 20.12 & & 1.27 & & -9.327 & 642.33 & \\
\hline $\mathrm{C} 03$ & SYBR & CyclA & Unkn & BepA1 basal & 18.78 & 18.84 & G03 & SYBR & IL-8 & Unkn & BepA1 basal & 20.66 & 20.30 & 1.88 & 1.57 & -8.717 & 420.78 & \\
\hline A04 & SYBR & CyclA & Unkn & BepA1 TNF-a & $17.40^{\prime}$ & & E04 & SYBR & IL-8 & Unkn & BepA1 TNF-a & $23.47^{\prime}$ & & 6.07 & & -4.530 & 23.10 & 29.42 \\
\hline B04 & SYBR & CyclA & Unkn & BepA1 TNF-a & 17.43 & & F04 & SYBR & IL-8 & Unkn & BepA1 TNF-a & 23.22 & & 5.79 & & -4.805 & 27.95 & \\
\hline $\mathrm{CO4}$ & SYBR & CyclA & Unkn & BepA1 TNF-a & 17.84 & 17.56 & G04 & SYBR & IL-8 & Unkn & BepA1 TNF-a & 23.22 & 23.30 & 5.38 & 5.75 & -5.217 & 37.21 & \\
\hline A05 & SYBR & CyclA & Unkn & FIC basal & $18.13^{\prime}$ & & E05 & SYBR & IL-8 & Unkn & FIC basal & $23.46^{\prime}$ & & 5.33 & & -5.263 & 38.41 & 36.36 \\
\hline B05 & SYBR & CyclA & Unkn & FIC basal & 18.25 & & F05 & SYBR & IL-8 & Unkn & FIC basal & 23.6 & & 5.35 & & -5.242 & 37.84 & \\
\hline C05 & SYBR & CyclA & Unkn & FIC basal & 18.38 & 18.25 & G05 & SYBR & IL-8 & Unkn & FIC basal & 23.94 & 23.67 & 5.56 & 5.42 & -5.036 & 32.81 & \\
\hline A06 & SYBR & CyclA & Unkn & FIC TNF-a & $18.40^{\prime}$ & & E06 & SYBR & IL-8 & Unkn & FIC TNF-a & $20.28^{\prime}$ & & 1.88 & & -8.715 & 420.24 & 430.61 \\
\hline B06 & SYBR & CyclA & Unkn & FIC TNF-a & 18.45 & & F06 & SYBR & IL-8 & Unkn & FIC TNF-a & 20.27 & & 1.82 & & -8.774 & 437.87 & \\
\hline $\mathrm{C} 06$ & SYBR & CyclA & Unkn & FIC TNF-a & 18.58 & 18.48 & G06 & SYBR & IL-8 & Unkn & FIC TNF-a & 20.42 & 20.32 & 1.84 & 1.85 & -8.761 & 433.72 & \\
\hline
\end{tabular}

Appendix figure 7.6.2 qPCR raw data and basic Livak analysis calculations are presented for IL-8. 


\begin{tabular}{|c|c|c|c|c|c|c|c|c|c|c|c|c|c|c|c|c|c|c|}
\hline Well & Fluor & Target & Content & Sample & Cq & $\begin{array}{l}\text { Mean Cq } \\
\text { (of } \\
\text { triplicates) }\end{array}$ & Well & Fluor & Target & Content & Sample & Cq & $\begin{array}{l}\text { Mean Cq } \\
\quad \text { (of } \\
\text { triplicates) }\end{array}$ & $\begin{array}{l}\text { Normalising } \\
\text { against } \\
\text { reference gene }\end{array}$ & average & $\begin{array}{l}\text { Normalising } \\
\text { against control }\end{array}$ & $\begin{array}{l}2^{\wedge}-\text { (colum } \\
\text { R) (Fold } \\
\text { change) }\end{array}$ & $\begin{array}{l}\text { Fold } \\
\text { change } \\
\text { average }\end{array}$ \\
\hline $\mathrm{A} 01$ & SYBR & CyclA & Unkn & HeLa basal & $19.35^{\prime}$ & & A01 & SYBR & SM22 & Unkn & HeLa basal & N/A & & & & & & \\
\hline B01 & SYBR & CycIA & Unkn & HeLa basal & 18.34 & & B01 & SYBR & SM22 & Unkn & HeLa basal & 22.32 & & 3.98 & & & & \\
\hline $\mathrm{C} 01$ & SYBR & CyclA & Unkn & HeLa basal & 18.61 & 18.77 & $\mathrm{C} 01$ & SYBR & SM22 & Unkn & HeLa basal & $N / A$ & 22.32 & & 3.98 & 0.00 & 1.0 & 1 \\
\hline $\mathrm{A} 02$ & SYBR & CyclA & Unkn & HeLa TNF- $\varepsilon$ & $\overrightarrow{18.30}$ & & A02 & SYBR & SM22 & Unkn & HeLa TNF-a & $21.17^{\prime}$ & & 2.87 & & -1.11 & 2.2 & \\
\hline B02 & SYBR & CycIA & Unkn & HeLa TNF- $\varepsilon$ & 18.25 & & B02 & SYBR & SM22 & Unkn & HeLa TNF-a & 21.05 & & 2.80 & & 0.00 & 1.0 & \\
\hline $\mathrm{C} 02$ & SYBR & CycIA & Unkn & HeLa TNF- $\varepsilon$ & 18.30 & 18.28 & $\mathrm{C} 02$ & SYBR & SM22 & Unkn & HeLa TNF-a & 21.02 & 21.08 & 2.72 & 2.80 & 0.00 & 1.0 & 1.4 \\
\hline $\mathrm{A} 03$ & SYBR & CyclA & Unkn & BepA1 bas $\varepsilon$ & $18.88^{\prime}$ & & A03 & SYBR & SM22 & Unkn & BepA1 basal & $23.57^{\prime \prime}$ & & 4.69 & & 0.71 & 0.6 & \\
\hline B03 & SYBR & CyclA & Unkn & BepA1 bas $\varepsilon$ & 18.85 & & B03 & SYBR & SM22 & Unkn & BepA1 basal & 23.31 & & 4.46 & & 0.48 & 0.7 & \\
\hline $\mathrm{CO3}$ & SYBR & CycIA & Unkn & BepA1 bas $\varepsilon$ & 18.88 & 18.87 & $\mathrm{CO3}$ & SYBR & SM22 & Unkn & BepA1 basal & 23.24 & 23.37 & 4.36 & 4.50 & 0.38 & 0.8 & 0.7 \\
\hline A04 & SYBR & CyclA & Unkn & BepA1 TNF & $17.30^{\prime}$ & & A04 & SYBR & SM22 & Unkn & BepA1 TNF-a & $N / A^{\prime \prime}$ & & & & & & \\
\hline B04 & SYBR & CycIA & Unkn & BepA1 TNF & 17.53 & & B04 & SYBR & SM22 & Unkn & BepA1 TNF-a & 23.29 & & 5.76 & & 1.78 & 0.3 & \\
\hline $\mathrm{CO} 4$ & SYBR & CyclA & Unkn & BepA1 TNF & 17.21 & 17.35 & $\mathrm{CO4}$ & SYBR & SM22 & Unkn & BepA1 TNF-a & 23.12 & 23.21 & 5.91 & 5.84 & 1.93 & 0.3 & 0.3 \\
\hline A05 & SYBR & CyclA & Unkn & FIC basal & $17.13^{\prime}$ & & A05 & SYBR & SM22 & Unkn & FIC basal & $22.28^{\prime}$ & & 5.15 & & 1.17 & 0.4 & \\
\hline B05 & SYBR & CyclA & Unkn & FIC basal & 17.25 & & B05 & SYBR & SM22 & Unkn & FIC basal & 22.93 & & 5.68 & & 1.71 & 0.3 & \\
\hline $\mathrm{C} 05$ & SYBR & CyclA & Unkn & FIC basal & 17.38 & 17.25 & $\mathrm{C} 05$ & SYBR & SM22 & Unkn & FIC basal & 22.92 & 22.71 & 5.54 & 5.46 & 1.56 & 0.3 & 0.4 \\
\hline A06 & SYBR & CyclA & Unkn & FIC TNF-a & $18.25^{\prime}$ & & A06 & SYBR & SM22 & Unkn & FIC TNF-a & N/A & & & & & & \\
\hline B06 & SYBR & CyclA & Unkn & FIC TNF-a & 18.25 & & B06 & SYBR & SM22 & Unkn & FIC TNF-a & 22.76 & & 4.51 & & 0.53 & 0.7 & \\
\hline $\mathrm{C} 06$ & SYBR & CyclA & Unkn & FIC TNF-a & 18.28 & 18.26 & $\mathrm{C} 06$ & SYBR & SM22 & Unkn & FIC TNF-a & 22.72 & 22.74 & 4.44 & 4.47 & 0.46 & 0.7 & 0.7 \\
\hline
\end{tabular}

Appendix figure 7.6.3 qPCR raw data and basic Livak analysis calculations are presented for SM22. 


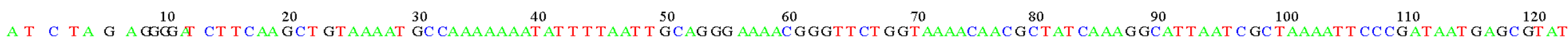

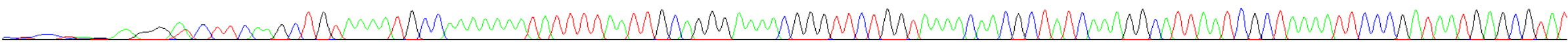

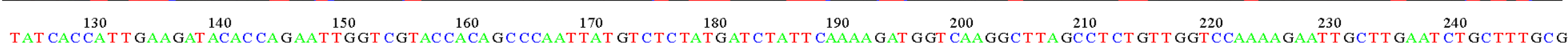

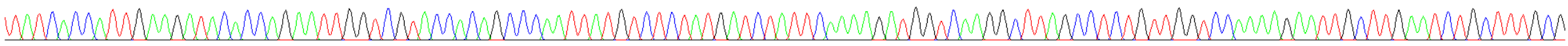

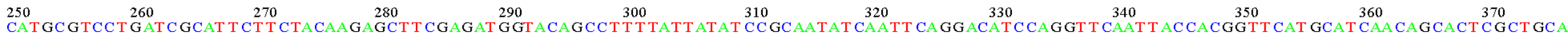

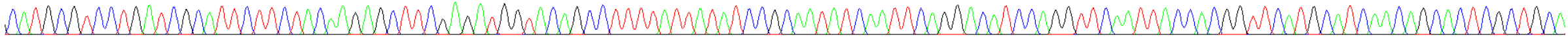

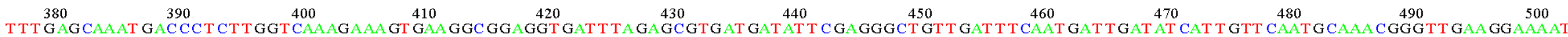

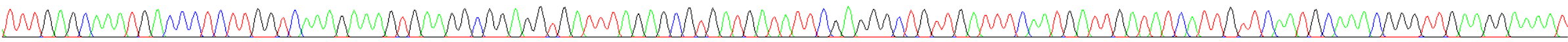

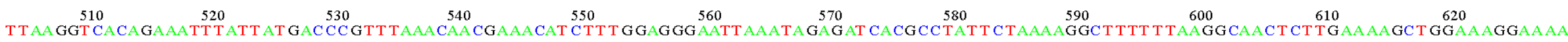




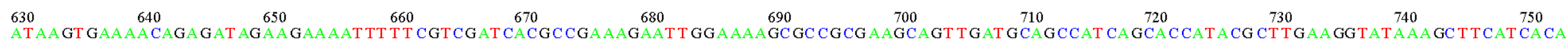

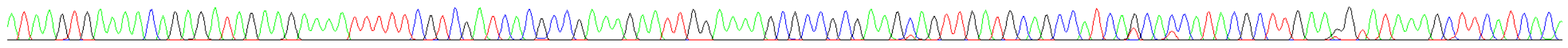
$\begin{array}{ccccccc}760 & 770 & 780 & 790 & 800 & 810 & 820\end{array}$

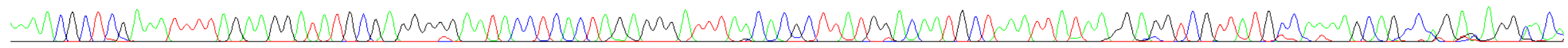

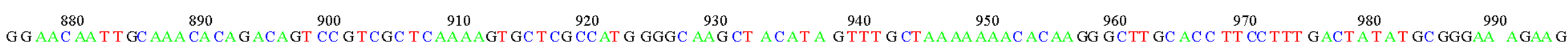

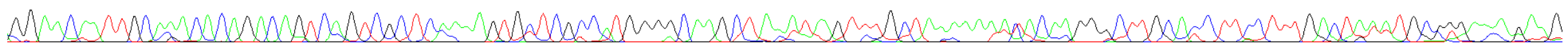

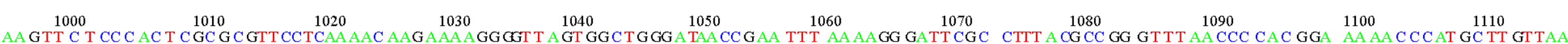

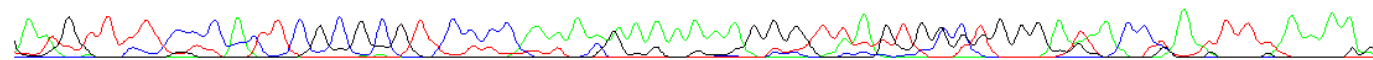

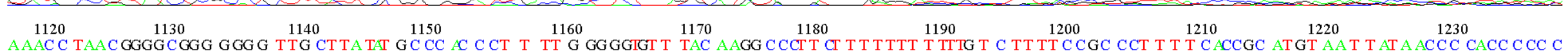

Appendix Figure 7.7 Sequencing data for the BepA1_BepA2 mutagenesis plasmid. The sequence peaks for the BepA1_BepA2 mutagenesis plasmid are shown. The insert was sequenced with both the M13F and M13R primers. The sequence shown here is from the M13F primer. 
